Check for updates

Cite this: RSC Adv., 2017, 7, 42242

Received 10th May 2017

Accepted 19th August 2017

DOI: $10.1039 / \mathrm{c} 7 \mathrm{ra05302a}$

rsc.li/rsc-advances

\section{Design and synthesis of coordination polymers with chelated units and their application in nanomaterials science}

\begin{abstract}
Gulzhian I. Dzhardimalieva and Igor E. Uflyand (DD ${ }^{\mathrm{b}}$
The advances and problems associated with the preparation, properties and structure of coordination polymers with chelated units are presented and assessed. The metalloligand strategy, and columnar-layered strategy, as well as approaches based on supramolecular building blocks and supramolecular building layers in the design of coordination polymers with chelated units are considered. The conventional (solvent evaporation, diffusion, hydro(solvo)thermal, ionothermal, microfluidic, surfactant-thermal, and in situ spacer methods as well as synthesis in supercritical $\mathrm{CO}_{2}$ ) and alternative (microwave-assisted, electrochemical, mechanochemical, and sonochemical methods) synthesis routes are described. Special attention is paid to the post-synthetic approaches to the preparation of coordination polymers with chelated units including post-synthetic modification, post-synthetic deprotection, and post-synthetic exchange. The thermal transformations of coordination polymers, resulting in the formation of nanocomposite materials, are considered. The bibliography includes the papers published within last five years.
\end{abstract}

${ }^{a}$ Laboratory of Metallopolymers, The Institute of Problems of Chemical Physics RAS, Academician Semenov Avenue 1, Chernogolovka, Moscow Region, 142432 Russian Federation. E-mail: dzhardim@icp.ac.ru
${ }^{b}$ Department of Chemistry, Southern Federal University, B. Sadovaya Str. 105/42, Rostov-on-Don, 344006 Russian Federation.E-mail: ieuflyand@sfedu.ru

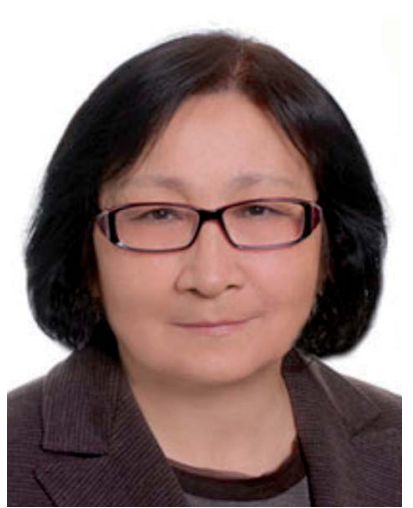

Gulzhian I. Dzhardimalieva, Dr. Sci. (Chem.), Ph.D. is the Head of Laboratory, Institute of Problems of Chemical Physics Russian Academy of Sciences and Professor of Department of Applied Mechanics, Moscow Aviation Institute (National Research University). She received her Ph.D. in 1987 from the Institute of Chemical Physics, RAS in Moscow, and her Doctorate in Chemistry in 2010 from Institute of Problems of Chemical Physics RAS in Chernogolovka. The scope of her scientific interest is: metal-containing monomers and polymers on their base, macromolecular metal carboxylates, coordination polymers, metallopolymer nanocomposites, and nanomaterials. Dr. Dzhardimalieva is author of about 150 articles and 4 monographs. She has been as invited researcher of Auburn University, USA (2001), Warsaw Technology University, Poland (2002, 2011), Bremen University, Germany (2007), Turin University, Italy (2008), and Institute of composite and biomedical materials CNR, Neapol, Italy (2011, 2013). She was issue editor of Journal of Inorganic and Organometallic Polymers and Materials (2016, Vol. 26, 6).

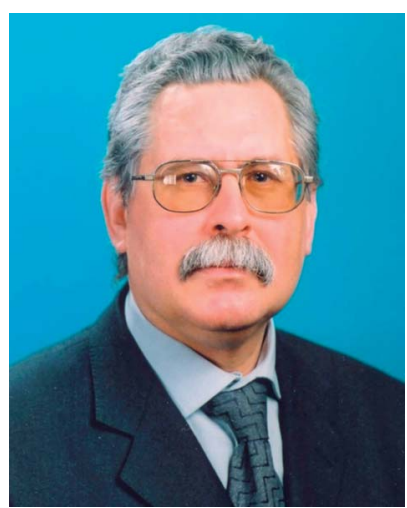

Prof. Igor E. Uflyand, Dr. Sci. (Chem.) is the Head of Chemistry Department, Southern Federal University, Rostov-onDon, Russia. He was born in 1956 and graduated from Rostov State University, Department of Physical and Colloid Chemistry. Prof. Uflyand received his Ph.D. in 1981 and his Doctorate in Chemistry in 1996 from the Rostov State University. He received the title of Professor in 1996. The scope of his scientific interest is: coordination and organometallic chemistry, metal chelate monomers and polymers on their basis, metal-containing polymers, catalysis by metal complexes, nanomaterials. Prof. Uflyand is the author of over 150 scientific papers, 10 patents and two books. 


\section{Introduction}

Coordination polymers (CPs) are objects of increasing interest to researchers. ${ }^{1-7}$ These advanced structures can be considered from two different points of view: only scientific knowledge or practical orientation. To date, owing to the efforts of specialists in various fields such as polymer, coordination, material, bioinorganic and analytical chemistry, photochemistry, catalysis, etc., considerable advances in this field of chemistry have been achieved. It is important that CPs are porous materials with adjustable size of pores, ${ }^{8}$ have vast surface areas and intriguing structural topologies. ${ }^{9-11}$ A great interest in the development of new CPs is associated with their promising applications, ${ }^{12}$ especially in such areas as ion exchange, adsorption, and separation processes, ${ }^{13-23}$ biomedical applications, ${ }^{24,25}$ drug delivery, ${ }^{26,27}$ sensor technologies, ${ }^{28,29}$ heterogeneous ${ }^{22,30-39}$ and biomimetic catalysis, ${ }^{\mathbf{4 0 , 4 1}}$ luminescence, ${ }^{\mathbf{2 6 , 2 8 , 4 2 - 4 4}}$ proton conductivity, ${ }^{22,40,41}$ etc.

In addition, CPs have gained more and more attention as nanocomposite materials precursors, and they serve not only as a starting material, but also as stabilizers of formed nanoparticles. ${ }^{4-52}$ From the view of the design of materials, solidstate thermolysis of CPs with different structures and compositions is a simple and rational route to synthesize novel nanostructured materials. Thermal decomposition of CPs under various conditions has been widely studied with the aim to prepare a variety of nanomaterials with desired sizes and morphologies. It is important that various nano-sized materials, for example, carbon, metals, metal oxides, etc., can be obtained using CP or their precursors.

Coordination polymers with chelated units are an interesting topic in the branch of coordination chemistry due to its specific properties and high stability than ordinary complexes due chelate or macrocyclic effect. They relate to a special class of polymeric metal chelates containing metal in the main chain, whose special feature is breaking a polymer backbone after a metal is removed. ${ }^{\mathbf{5 3 , 5 4}}$

The present review aims to describe the latest advances in the field of the coordination polymers with chelated units including main strategies and approaches to their design as well as synthetic methodologies of their preparation. It contains the basic directions of using coordination polymers as precursors of nanostructured materials, a conclusion and the main challenges.

\section{Main strategies and approaches}

To date, a wide variety of strategies and approaches to the design of coordination polymers with chelated units have been developed. Among them, metalloligand and pillar-layered strategies as well as approaches based on supramolecular building blocks and layers are the most common.

\subsection{Metalloligand strategy}

Though a great amount of building units has brought to vast variety of target materials, using well-defined metal complex as a building unit (i.e. metalloligand) is unique for building projected architecture. ${ }^{55} \mathrm{CP}$ assembling based on metalloligand approach proceeds mainly in two stages: (1) synthesis of a metalloligand by interaction between well-defined ligands and metal ions (mostly 3d metal ions), and (2) reaction of the metalloligand with a second type of a metal ion, which plays a role of nodal units in a framework. The metalloligand used as a building unit provides structural rigidity, which puts ancillary functional groups in predetermined conformation. These ancillary functionalities can then coordinate a secondary metal ion and take part in self-assembling through such weak intermolecular interactions as H-bonds. Two classes of metalloligands are respectively distinguished, which have competitive $\mathrm{M}$-L-bond sensitive groups, and $\mathrm{H}$-bond sensitive functional groups. It is this that metalloligand differs from a simple complex in which the metal ion is coordinated with a certain number of "ordinary ligands" that do not contain an attached functional group. It is important that both metal complexes with monofunctional ligands and metal chelates can act as a metalloligand. The latter constitute the main group of metalloligands used to create $\mathrm{CP}$ with chelated units.

Among metalloligands, the widest applied as building units are metalloporphyrins (M-Pp). ${ }^{56-75}$ By integration of different functional peripheral substituents into $\beta$-pyrrol and/or mesopositions, M-Pp can have several multifunctional properties. With M-Pp used as building units for metal-organic frameworks (MOFs), easy molecular modification of Pp widens possibilities of structural design. Since most elements of periodic table can be included in Pp, different metal centers can be integrated into porous surface of MOFs without changing a framework topology. Moreover, using M-Pp as building units for MOFs gives an opportunity to adapt photochemical, redox, catalytic, and other properties of frameworks.

Most widely used Pp-based ligands for CPs building are tetrakis(4-carboxyphenyl)-Pp $\left(\mathrm{L}^{1}\right)$ and tetrakis(4-pyridyl)-Pp $\left(\mathrm{L}^{2}\right)$. The various multiporphyrin frameworks with different topology and dimensionality are built on the basis of coordination of their peripheral carboxyphenyl or pyridine fragments. Therefore, $\mathrm{L}^{1}$ and $\mathrm{L}^{2}$ are almost inexhaustible sources for building either H-bonding or M-L-driven CPs. It should be noted that for design of Pp-based MOFs, clusters formed by rigid Lewis acid metals, such as $\mathrm{Zr}_{6}, \mathrm{Hf}_{6}, \mathrm{Zr}_{8}, \mathrm{Hf}_{8}$ and $\mathrm{Fe}_{3}$, are used as most stable secondary building unit (SBUs). ${ }^{76}$ Thus, systematic study of $\mathrm{Zr}$ - and Hf-Pp MOFs has brought to discovery of isostructural PCN-221(M) based on metalloligands and $\mathrm{Zr}_{8}$ or $\mathrm{Hf}_{8}$ clusters. ${ }^{65}$ In particular, cluster $\mathrm{Zr}_{8} \mathrm{O}_{6}$ is characterized by idealized $\mathrm{Zr}_{8}$ cube, in which each $\mathrm{Zr}$ atom is in one vertex and each face of the cube is crowned with one $\mu_{4}$-oxygen atom. On each face of the cube carboxylate from $\mathrm{Pp}$ ligands bridges two $\mathrm{Zr}$ atoms thus obtaining 3D MOF with very rare $(4,12)$-connected ftw topology, in which two types of polyhedral cages with diameters $\sim 1.1$ and $\sim 2.0 \mathrm{~nm}$ and cage opening $\sim 0.8 \mathrm{~nm}$ are found. As another example, we shall notice a solvothermal reaction of $\mathrm{Fe}-\mathrm{L}^{1}, \mathrm{ZrCl}_{4}$ and benzoic acid in DEF during $48 \mathrm{~h}$ at $120{ }^{\circ} \mathrm{C}$, which leads to PCN-222(Fe) with a framework consisting of square-planar Pp ligands linked to $\mathrm{Zr}_{6}$ clusters. ${ }^{56}$ In this $\mathrm{CP}$, each Fe-L ${ }^{1}$ block is coordinated with four 8-connected clusters $\mathrm{Zr}_{6}$ with twisted 


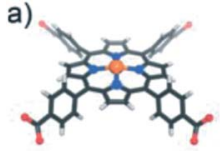

III

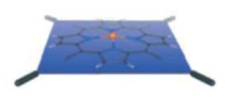

d)

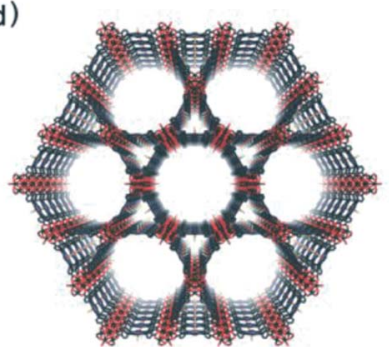

b)

III

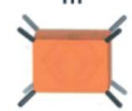

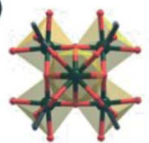

e)

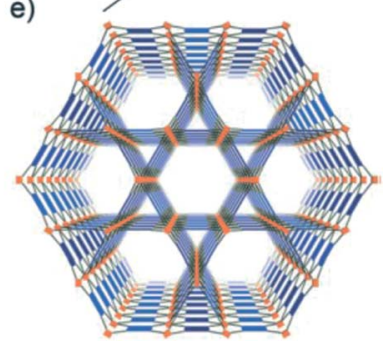

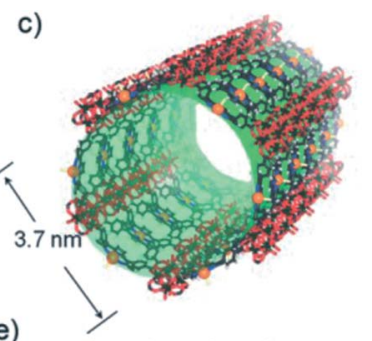

Fig. 1 The crystal structure and underlying network topology of PCN$222(\mathrm{Fe})$. The Fe- $\mathrm{L}^{1}$ (a; blue square) is connected to four 8-connected $\mathrm{Zr}_{6}$ clusters (b; light orange cuboid), generating a 3D network with Kagome-like topology (d, e) and large 1D channels (c; green pillar). Zr: black spheres, C: gray, O: red, N: blue, and Fe: orange. [Reprinted with permission from ref. 56. Copyright 2012, Wiley-VCH Verlag GmbH \& Co. KGaA, Weinheim.] angle forming 3D network with Kagome-like topology (Fig. 1). It is remarkable that the mesoporous framework contains large 1D hexagonal open channels with a larger diameter $3.7 \mathrm{~nm}$ ranking among the largest for MOFs. ${ }^{7,78}$ In addition, this framework is preserved even after immersion in concentrated $\mathrm{HCl}$, which is rare in MOF materials. We shall also notice highly stable MOFs with 3D nanochannels PCN-224(Ni, Co, Fe) assembled with six-connected $\mathrm{Zr}_{6}$ cluster and $\mathrm{M}-\mathrm{L}^{1}$ metalloligands. ${ }^{79}$ The PCN-224 series not only demonstrates high BET surface area $\left(2600 \mathrm{~m}^{2} \mathrm{~g}^{-1}\right)$, but also stays unchanged at $\mathrm{pH}$ of aqueous solution from 0 to 11 .

Metal-metalloporphyrin frameworks (MMPFs) form a class of coordination networks self-assembled using reasonable choice of metal-containing SBUs and specially developed M-Pp metalloligands. ${ }^{80}$ As an example of CP of MMPF series, we shall consider MMPF- 6 based on $\mathrm{FeL}^{1}-\mathrm{Cl}$ metalloligand and SBU $\mathrm{Zr}$ oxide cluster, which is obtained under solvothermal conditions (Fig. 2). ${ }^{57}$

Porous MMPFs including $\left[\mathrm{Zn}_{2}(\mathrm{HCOO})_{2}\left(\mathrm{MnL}^{1}\right)\right], \quad\left[\mathrm{Cd}_{2^{-}}\right.$ $\left.(\mathrm{HCOO})_{2}\left(\mathrm{MnL}^{1}\right)\right], \quad\left[\mathrm{Zn}_{2}(\mathrm{HCOO})\left(\mathrm{FeL}^{1}\right)\right]$ and $\left[\mathrm{Cd}_{3}\left(\mathrm{H}_{2} \mathrm{O}\right)_{6}\left(\mu_{2^{-}}\right.\right.$ $\mathrm{O})\left(\mathrm{FeL}^{1}\right)_{2}$ ] units are synthesized by heating the mixture $\mathrm{MCl}-\mathrm{L}^{1}$ $(\mathbf{M}=\mathrm{Mn}$ and $\mathrm{Fe})$ and nitrates $\mathbf{M}^{\prime}\left(\mathbf{M}^{\prime}=\mathrm{Zn}\right.$ or $\left.\mathrm{Cd}\right)$ in mixed solvent from DMF and acetic acid. ${ }^{81}$ First three compounds are built from $\mathrm{M}_{2}^{\prime}(\mathrm{COO})_{4}$ paddlewheel subunits linked with $\mathrm{ML}^{1}$ bridge and formate ligands for formation of their $3 \mathrm{D}$

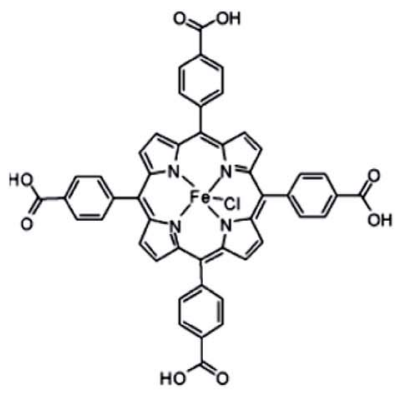

(a)

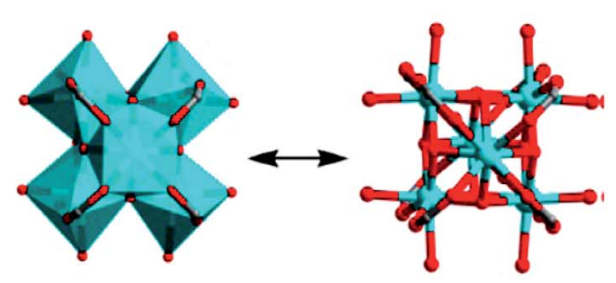

(b)

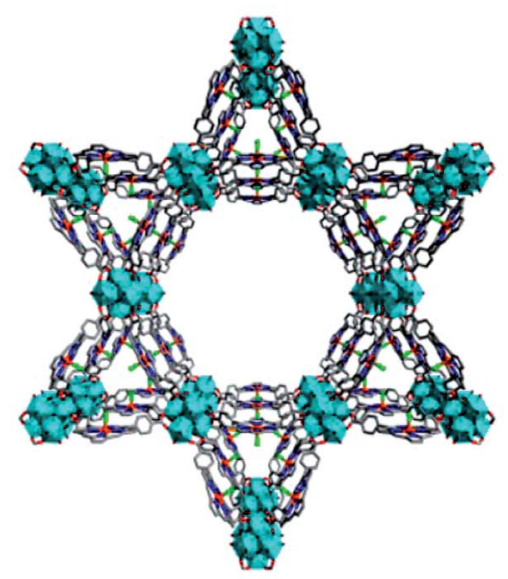

(c)

Fig. 2 (a) FeL ${ }^{1}-\mathrm{Cl}$ metalloligand. (b) $\mathrm{Zr}_{6} \mathrm{O}_{8}\left(\mathrm{CO}_{2}\right)_{8}\left(\mathrm{H}_{2} \mathrm{O}\right)_{8} \mathrm{SBU}$. (c) Hexagonal and triangular 1D channels of MMPF-6. Color scheme: $\mathrm{C}$, gray; O, red; Cl, green; Zr, turquoise. [Reprinted with permission from ref. 57. Copyright 2012, American Chemical Society.] 
a)

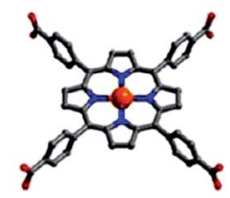

d)

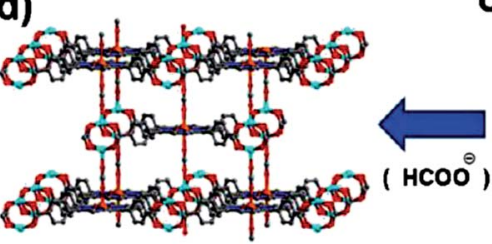

b)
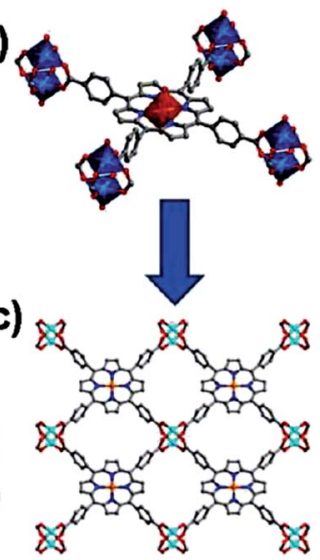

Fig. 3 (a) Deprotonated Mn-Pp ligand, (b) view of the coordination mode of $\mathrm{Mn}-\mathrm{Pp}$ and the coordination environments of $\mathrm{M}^{\prime}$ and $\mathrm{Mn}$ atoms, (c) lamellar network of $\mathrm{Mn}-\mathrm{Pp}$ linking up $\mathrm{M}_{2}^{\prime}(\mathrm{COO})_{4}$ paddlewheel SBUs, (d) perspective view of the 3D Pp framework. Color codes: $M^{\prime}$, cyan or light-blue square pyramids; $M n$, orange; $O$, red; $N$, blue; C, gray. [Reprinted with permission from ref. 81. Copyright 2013, American Chemical Society.]

connectivity. Formate column is heterogeneously connected with $\mathrm{M}$ and $\mathrm{M}^{\prime}$ cations in two first $\mathrm{CPs}$ and links homogeneously $\mathrm{M}^{\prime}$ cations in third CP. A $\mu_{2}-\mathrm{O}$ bridging $\mathrm{FeL}^{1}$ dimer plays a role of decadentate ligand for linking of $10 \mathrm{Cd}$ cations with formation of interesting 3D coordination network of fourth CP (Fig. 3).

The reaction of $\mathrm{M}-\mathrm{L}^{1}(\mathrm{M}=\mathrm{Co}, \mathrm{Ni}, \mathrm{Cu}, \mathrm{VO})$ with lead nitrate gives MOFs with $\left[\mathrm{Pb}_{2}\left(\mathrm{M}-\mathrm{L}^{1}\right)\right]$ units, which differ in framework topologies and $\mathrm{Pp}$ configurations changing from flat to wavy to bowl-shaped. ${ }^{82}$ It is important that Pp cores play pivotal role in the formation of different coordination frameworks by controlling Pp stacking, $\mathrm{L}^{1}$ coordination modes and the coordination spheres of the $\mathrm{Pb}(\mathrm{II})$ cations.

We should also notice CZJ-1 with $\left[\mathrm{Zn}_{2}\left(\mathrm{MnOH}-\mathrm{L}^{1}\right)\left(\mathrm{L}^{\prime}\right)\right]$ unit, where $L^{\prime}=N, N^{\prime}$-di(4-pyridyl)-1,4,5,8-naphthalenetetracarboxydiimide.$^{83}$ This porous MOF has a type of twice interpenetrated cubic $\alpha$-Po topology, in which $\mathrm{Zn}_{2}(\mathrm{COO})_{4}$ paddlewheel clusters are bridged by $\mathrm{MnOH}-\mathrm{L}^{1}$ in $2 \mathrm{D}$ sheets additionally linked by bridging organic pillared spacer $\mathrm{L}^{\prime}$ with formation of 3D porous structure.

Among other used Pp ligand, we shall notice 5,15bis(dicarboxyphenyl)-Pp. Thus, for example, frameworks with $\left[\mathrm{Cu}_{2}(\mathrm{ML})\right]_{n}$ unit have been obtained from ML metalloligands, where $\mathrm{M}=\mathrm{Zn}, \mathrm{Ni}, \mathrm{Pd}, \mathrm{MnCl}, \mathrm{Ru}(\mathrm{CO}),{ }^{84}$ using two different methods: interaction between $\mathrm{Cu}$ nitrate and $\mathrm{ML}$ in DMF at $80^{\circ} \mathrm{C}$ during $24 \mathrm{~h}$ or by diffusion of triethylamine vapor into the abovementioned mixture. It is interesting that this framework forms 3D network consisting of two-component combination $\left(\mathrm{Cu}_{2}\right.$ paddlewheel nodes and $\mathrm{ZnL}$ building units) and having internal spherical cavity of $20 \AA$ in diameter. This interior cavity is surrounded totally by 16 available metal centers of two different metals, i.e. with eight $\mathrm{Zn}$ atoms from $\mathrm{ZnL}$ units and eight $\mathrm{Cu}$ atoms from paddlewheel units (Fig. 4).

In another interesting example, narrowly distributed nanoand micro-meter CPs are obtained from Mn(III)-L metalloligand and Co acetate, in which morphologies are diversified from amorphous spheres in crystalline cubes through different periods of reaction (Fig. 5). ${ }^{85}$

In order to develop CPs based on Pp ligands with a possibility to coordinate more metal centers, more than four carboxyl or Py groups were involved as, for example, in octatopic Ppligand $\quad 5,10,15,20$-tetrakis(3,5-biscarboxyl-phenyl)-Pp. ${ }^{60,62}$ In particular, it is used for building of three porous M-Pp frameworks ZJU-18, ZJU-19 and ZJU-20, ${ }^{62}$ whose structures are 3-periodical, binodal, edge-transitive tbo networks (Fig. 6) showing intercrossed porous windows of about $11.5 \AA$ and porous cavities of about $21.3 \AA$ in diameter. These three isostructural MOFs were synthesized by heating $\mathrm{Mn}$ (III)Cl-L and $\mathrm{MnCl}_{2}, \mathrm{NiCl}_{2}$ or $\mathrm{CdCl}_{2}$ mixture, respectively, in mixed solvent from DMF and acetic acid at $80{ }^{\circ} \mathrm{C}$ during a week.

We shall also notice MOF based on tetrazolyl-Pp with $\left\{[\mathrm{Mn}(\mathrm{II})]_{4} \mathrm{Cl}[\mathrm{Mn}(\mathrm{III}) \mathrm{Cl}-\mathrm{L}]_{2}\right\}$ unit, called UTSA-57, which is built from $\mathrm{Mn}_{4} \mathrm{Cl}(\mathrm{L})_{8}\left(\mathrm{H}_{2} \mathrm{O}\right)_{4}$ clusters, linked with metalloligand $\mathrm{Mn}(\mathrm{III}) \mathrm{Cl}-\mathrm{L}$ (Scheme 1) ${ }^{86}$ UTSA-57 has a rare scu MOF topology and is built of 8-connected bridging SBUs with 1D square (a)

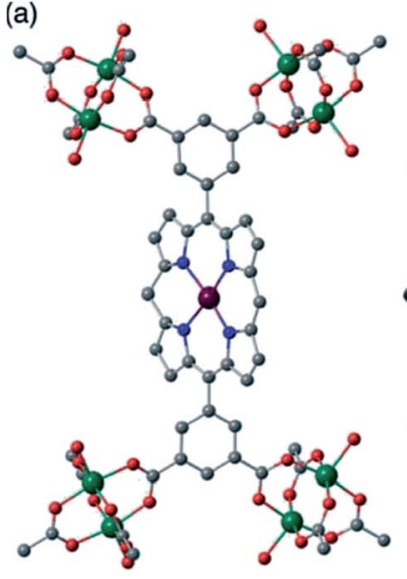

(b)

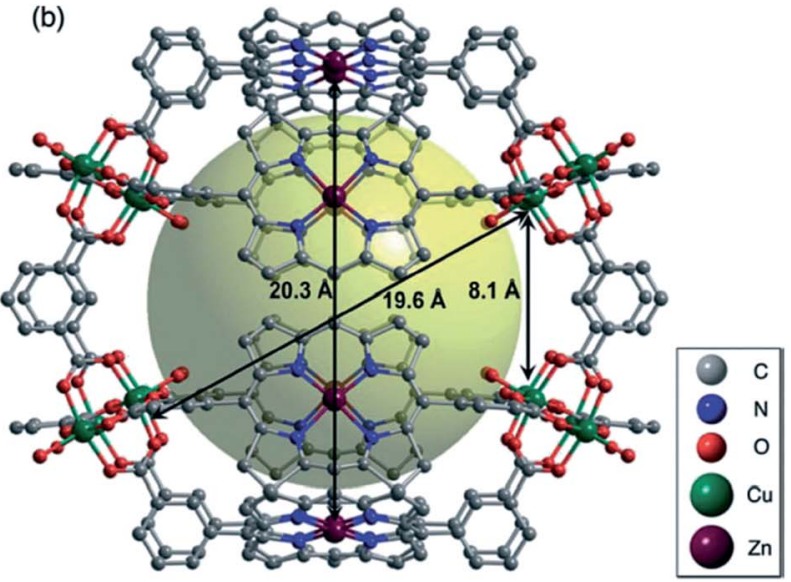

Fig. 4 (a) $A \mathrm{ZnL}$ moiety of $\left[\mathrm{Cu}_{2}(\mathrm{ZnL})\right]$; and (b) a cage consisting of eight $\mathrm{ZnL}$ ligands and eight paddlewheel $\mathrm{Cu}_{2}$ nodes. [Reprinted with permission from ref. 84. Copyright 2012, Wiley-VCH Verlag GmbH \& Co. KGaA, Weinheim.] 


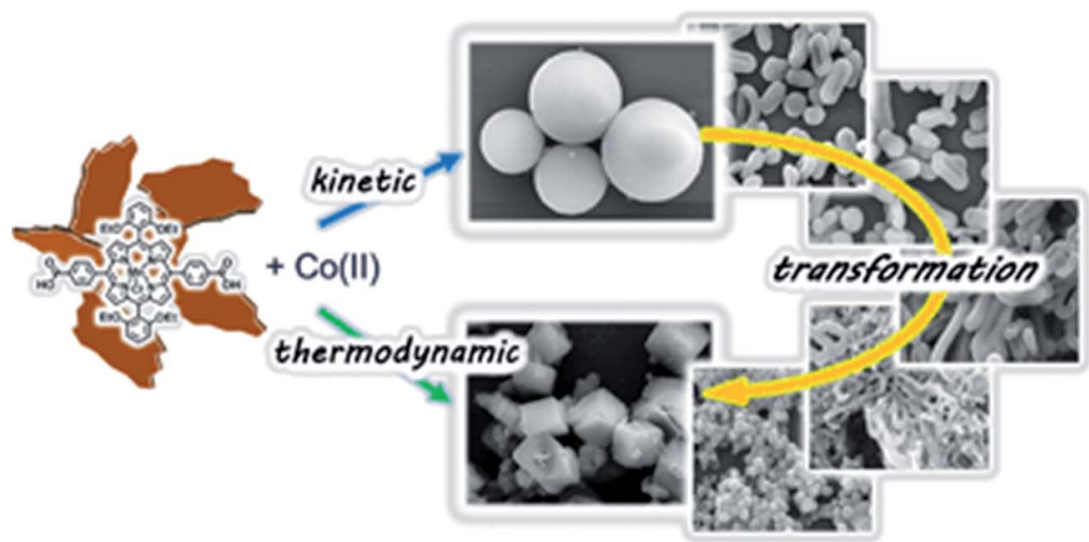

Fig. 5 A series of narrowly dispersed nano- and micro-sized CPs prepared from a $\mathrm{Mn}(\mathrm{III})-\mathrm{Pp}$ metalloligand acid and $\mathrm{Co}(\mathrm{OAC})_{2}$, which morphologies diversified from amorphous spheres to crystalline cubes by varying reaction periods. [Reproduced from ref. 85 with permission from The Royal Society of Chemistry.]

nanotube-like $20 \AA ̊$ channels along $c$ axis. In addition, UTSA-57 presents 3D stable microporous structure.

An example of metalloligand for MOF formation through $\mathrm{H}-$ bonds can be $\mathrm{Co}(\mathrm{II})-5,10,15,20$-tetra(4-(4-acetateethyl)phenoxy) phenyl-Pp. ${ }^{87}$ In it, M-Pp molecules are linked with each other by intermolecular $\mathrm{H}$-bonds with formation of $2 \mathrm{D}$ layer, which is additionally linked by $\pi \cdots \pi$ interactions for formation of $3 \mathrm{D}$ supramolecular structure having one type of micropores with $3.98 \times 6.47 \AA^{2}$. MOF has a permanent porosity with Langmuir surface area of $158.79 \mathrm{~m}^{2} \mathrm{~g}^{-1}$ and BET surface area of $97.70 \mathrm{~m}^{2} \mathrm{~g}^{-1}$. In another example, M-Pp coordination framework $\left[\mathrm{Co}\left(\mathrm{H}_{3} \mathrm{~L}\right)\right]_{n}$, where $\mathrm{H}_{6} \mathrm{~L}=$ meso-tetra(4-carboxyphenyl)-Pp, having 2D layered coordination network structure shows $\mathrm{H}$-bonds between carboxyl groups within 2D layer as well as between neighboring layers.

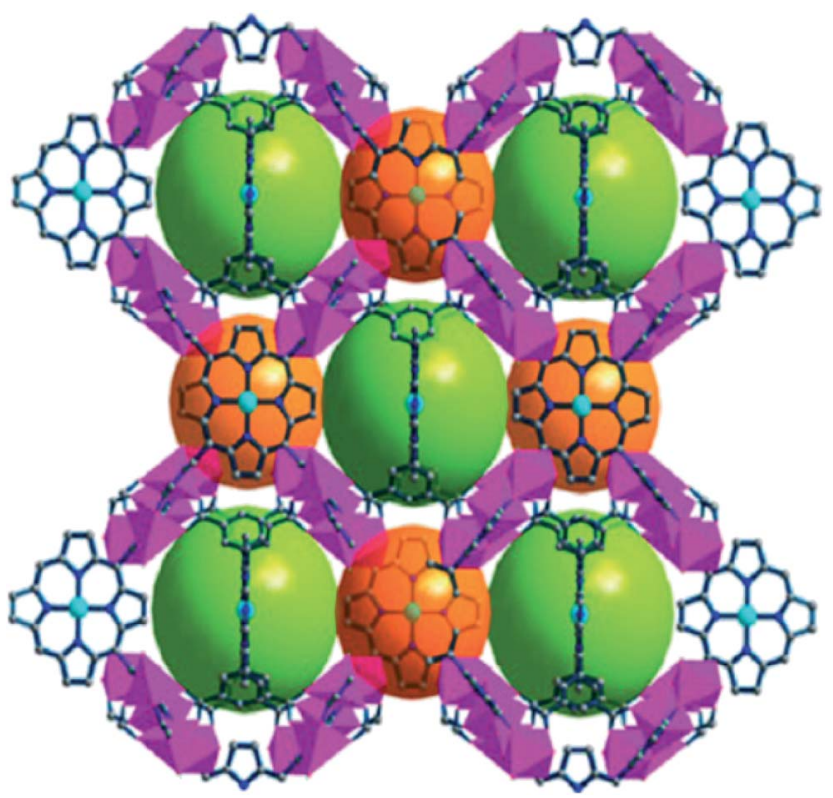

Fig. 6 The porous 3D crystal structure of ZJU-18. [Reprinted with permission from ref. 62. Copyright 2012, American Chemical Society.]
The popular metalloligands are metallosalens (salen is $N, N^{\prime}$ bis(salicylidene)ethylenediamine), ${ }^{88}$ in which additional functional groups, such as carboxylate, pyridine and benzoategroups are in para- and meta-positions with respect to $\mathrm{OH}^{-}$ groups on aromatic ring. As an example, it can be noted using solvothermal synthetic strategy for CPs building based on metallosalens as spacers. ${ }^{89}$ In this strategy, a metalloligand based on carboxy-functionalized salen spacer 2,6-bis[(4-carboxyanilino)carbonyl] pyridine and a metal salt are solved in DMSO/ DMF mixture, and then heated, which leads to CPs formation with governed size from nano- to micro-meter level. The ligand chelates metal cations in the center of the structure and provides two terminal carboxylate groups for $\mathrm{CP}$ growth. The particle size is regulated by changing the reaction temperature, so that a temperature increasing leads to bigger particles. Initially formed nanowires aggregate with formation of cub-like clusters, which undergo intrastructural fusion into uniform cubic-shaped particles. In addition, the size of the formed structures is influenced by DMSO/DMF ratio, at that higher content of DSMO leads to bigger cubes.

A special attention should be given to the metalloligand strategy in synthesis of heterometallic CPs, since used metalloligand already containing ion of an initial metal fits ideally for interaction with respective secondary metal ions. ${ }^{89-91}$ In conventional synthesis using the reaction between mixture of metal salts and organic ligands, it is very difficult to control synthesis of heterometallic complexes, since there is always high probability of formation of homometallic polynuclear complexes. At the same time, when metalloligand strategy is used, side reaction is minimal, and exclusively heterometallic $\mathrm{CP}$ can be obtained. A great number of metalloligands is developed, which provide targeted production of ordered heterometallic architectures. ${ }^{93-99}$ Interesting heterometallic $\mathrm{Cu}$ (II)$\mathrm{Zn}$ (II) CPs $2 \mathrm{~A}$ and 2B are synthesized by interaction of $\mathrm{CuL}$ metalloligand, where $\mathrm{H}_{2} \mathrm{~L}=N, N^{\prime}$-bis(salicylidene)-1,3propanediamine, with $\mathrm{Zn}$ perchlorate and sodium dicyanamide (dca) using different molar ratios of reagents (Scheme 2). ${ }^{100}$ The obtained compounds are 2D and 3D CPs, 

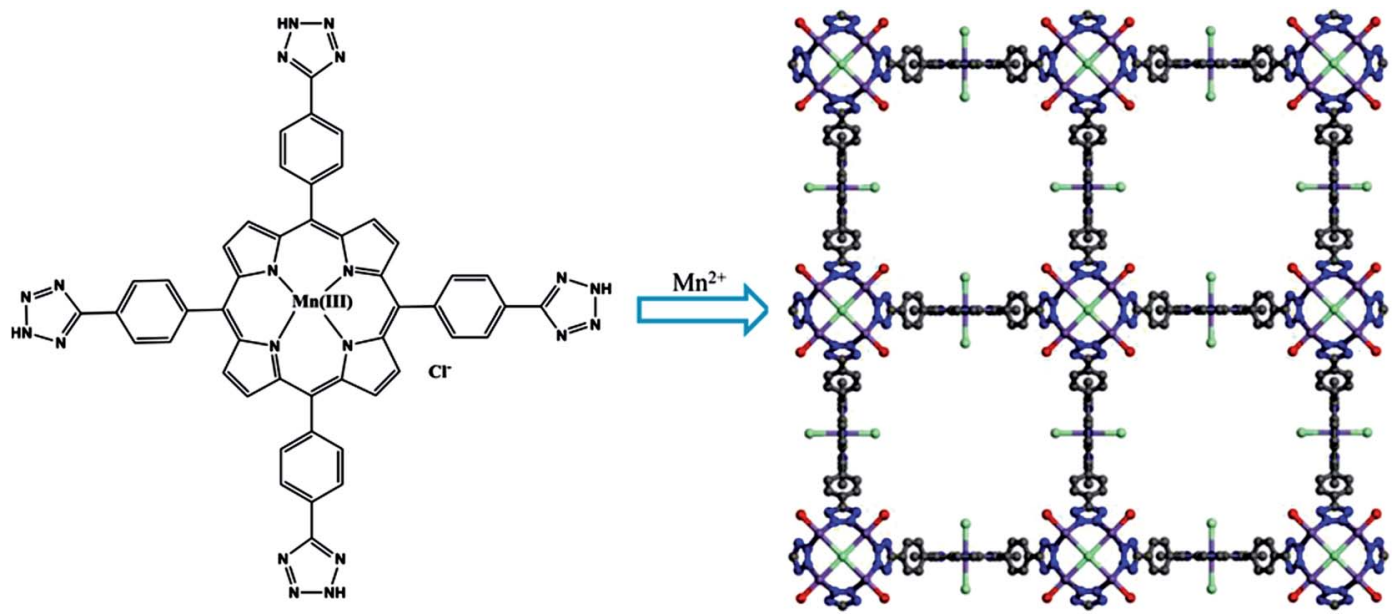

Scheme 1 A representation of processes from metalloligand $\mathrm{Mn}(\mathrm{II}) \mathrm{Cl}-\mathrm{L}$ to a 3D crystal structure of UTSA-57. [Reprinted with permission from ref. 86. Copyright 2015, American Chemical Society.]

respectively, with a common $\left[(\mathrm{CuL})_{2} \mathrm{Zn}\left(\mathrm{N}(\mathrm{CN})_{2}\right)_{2}\right]$ trinuclear unit, in which dea fragments have $\mu_{1,5}$-bridging coordination regime. It is interesting that the isomeric CPs is a rare example of 《henuine supramolecular isomerism $\rangle$. It should be noted that the reaction of similar $\left[\mathrm{CuL}^{1}\right]$ metalloligand (where $\mathrm{H}_{2} \mathrm{~L}^{1}$ is $N, N^{\prime}$-bis( $\alpha$-methylsalicylidene)-1,3-propanediamine) with zinc perchlorate hexahydrate and sodium dicyanamide, in a $2: 1: 2$ molar ratio at room temperature, resulted in a heterometallic discrete trinuclear complex, $\left[\left(\mathrm{CuL}^{1}\right)_{2} \mathrm{Zn}\left(\mathrm{N}(\mathrm{CN})_{2}\right)_{2}\right](\mathbf{1})$.

$\mathrm{Cu}(\mathrm{I})$-salen CPs $\left\{[\mathrm{NiL}]_{2}[\mathrm{Cu}(\mathrm{I}) \mathrm{CN}]_{9}\right\}_{n}, \quad\left\{[\mathrm{Cu}(\mathrm{II}) \mathrm{L}]_{2}[\mathrm{Cu}(\mathrm{I}) \mathrm{CN}]_{9}\right\}_{n}$ and $\left\{[\mathrm{NiL}][\mathrm{Cu}(\mathrm{I}) \mathrm{I}]_{2}\right\}_{n}$ are obtained by direct linking metallosalen precursors with $[\mathrm{Cu}(\mathrm{I}) \mathrm{CN}]_{n}$ chains and $\mathrm{Cu}_{2} \mathrm{I}_{2}$ clusters using the metalloligand strategy (Scheme 3). ${ }^{101}$ In these CPs, efficient integration of catalytically active transition metals of $\mathrm{Ni}(\mathrm{II}) /$ $\mathrm{Cu}(\mathrm{II})$ (salen) units and photoactive $\mathrm{Cu}(\mathrm{I})$ species in one solid

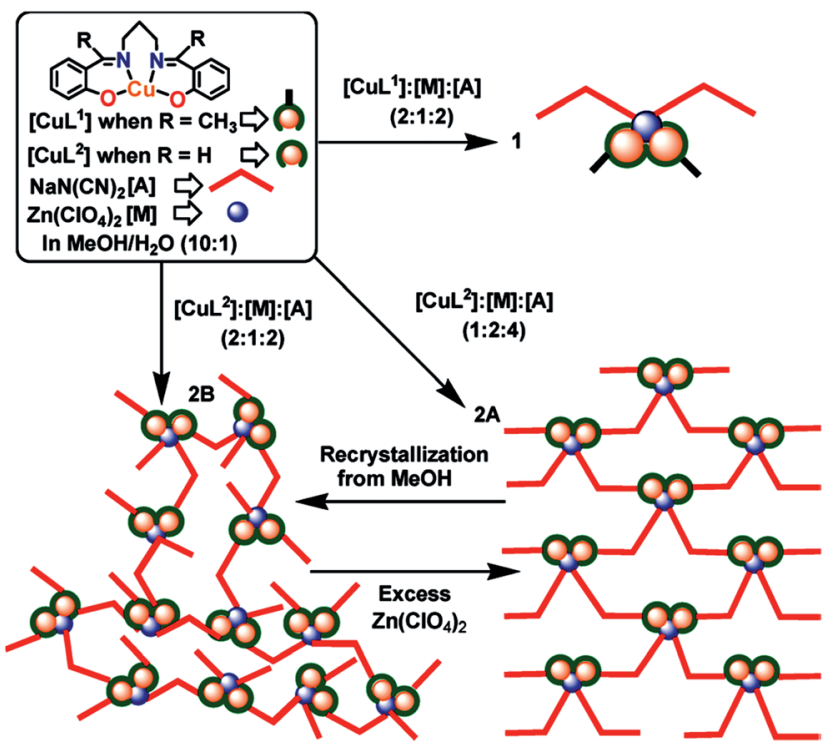

Scheme 2 Formation of the compounds 1, 2A and 2B. [Reproduced from ref. 100 with permission from The Royal Society of Chemistry.] polymer is achieved, which makes it possible to obtain materials with double catalytic properties.

Heterometallic CPs based on metallosalens have shown their efficiency in a range of catalytic processes, in particular, Mn(II)/ $\mathrm{Cu}(\mathrm{II})$-salen MOFs in separation of chiral and achiral small molecules, ${ }^{91} \mathrm{Mn}$ (II)/Ni(II)-salen 1D CPs in olefin epoxidation, ${ }^{90}$ $\mathrm{Cu}(\mathrm{II}) / \mathrm{Cu}(\mathrm{II})$-salen-based $1 \mathrm{D} \mathrm{CP}$ in three-component Strecker reaction. ${ }^{102}$

Wide range of metalloligands is presented by acaccomplexes, which are used, for example, in synthesis of heterometallic CPs with $\left[\mathrm{NiAg}_{3}(\mathrm{acac})_{3}\left(\mathrm{NO}_{3}\right)_{2}\right]_{n},\left[\mathrm{NiAg}_{3}(\mathrm{acac})_{3}\left(\mathrm{ClO}_{4}\right)_{2}\right]_{n}$ and $\left[\mathrm{NiAg}_{2}(\mathrm{acac})_{2}\left(\mathrm{CF}_{3} \mathrm{SO}_{3}\right)_{2}\right]_{n}$ units, where acac is acetylacetonate anion, through integration of respective $\mathrm{Ag}$ salts into the reaction with $\mathrm{Ni}(\mathrm{acac})_{2}$ metalloligand (Scheme 4). ${ }^{103}$ It is important that anions in CPs are decisive factors for formation of different structures. For example, first $\mathrm{CP}$ is outstanding 3D chiral framework, second $\mathrm{CP}$ has 2D inorganic layers, and third CP shows 2D (4,5)-connected layered architecture.

We shall notice crystal engineering of heterometallic CPs based on $\mathrm{FeL}_{3}$ and $\mathrm{Fe}\left(\mathrm{L}^{\prime}\right)_{3}$ chelates, where $\mathrm{L}=1,3$-di(4-pyridyl) propane-1,3-dionato and $\mathrm{L}^{\prime}=1,3$-di(3-pyridyl)propane-1,3dionato, which act as metalloligands in reactions with Ag salts

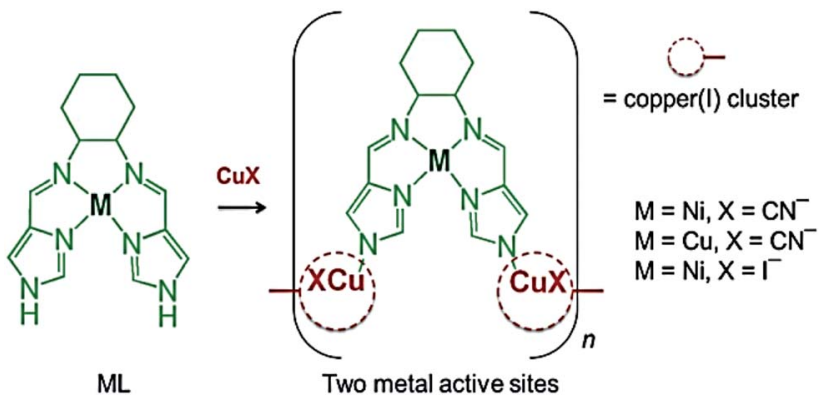

Scheme 3 Synthesis of $\left\{[\mathrm{NiL}]_{2}[\mathrm{Cu}(I) \mathrm{CN}]_{9}\right\}_{n},\left\{[\mathrm{Cu}(I) \mathrm{L}]_{2}[\mathrm{Cu}(\mathrm{I}) \mathrm{CN}]_{9}\right\}_{n}$ and $\left\{[\mathrm{NiL}][\mathrm{Cu}(\mathrm{I})]_{2}\right\}_{n}$. [Reproduced from ref. 101 with permission from The Royal Society of Chemistry.] 


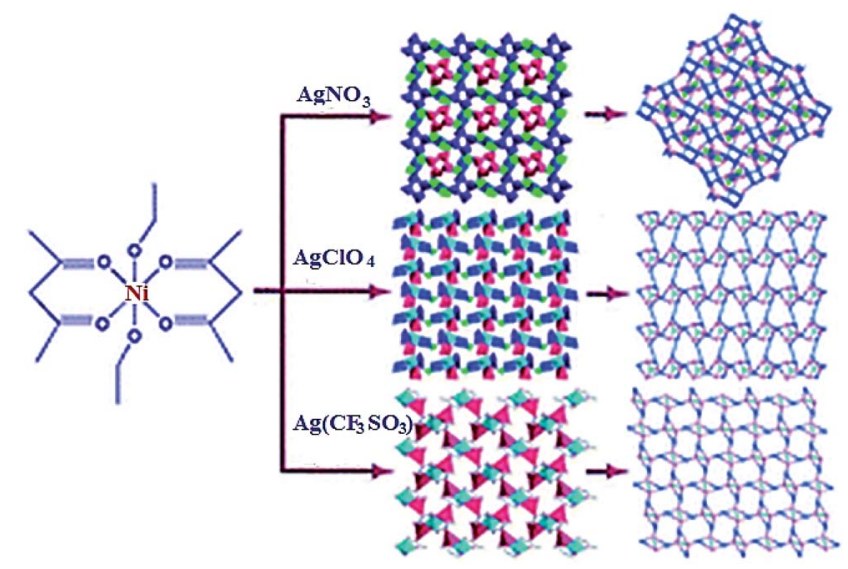

Scheme 4 The assembly of three $\mathrm{Ag}-\mathrm{Ni}$ heterometallic CPs. [Reproduced from ref. 103 with permission from The Royal Society of Chemistry.]

forming CPs with $\left[\mathrm{AgFeL}_{3}\right]_{n}$ and $\left[\mathrm{AgFe}\left(\mathrm{L}^{\prime}\right)_{3}\right]_{n}$ units (Scheme 5). ${ }^{104}$ It is important that structure of obtained CPs depends on nature of used counter-ions. Thus, reaction between $\mathrm{FeL}_{3}$ and $\mathrm{AgBF}_{4}$ leads to $\mathrm{CP}$, in which $\mathrm{Ag}$ centers link metalloligands in discrete nanotubes, while the reactions with $\mathrm{AgPF}_{6}$ and $\mathrm{AgSbF}_{6}$ have given CPs, in which metalloligands are bound in sheets. At the same time, interaction between $\mathrm{Fe}\left(\mathrm{L}^{\prime}\right)_{3}$ and $\mathrm{AgNO}_{3}$ leads to layered $\mathrm{CP}$ with $\left[\mathrm{Ag}_{2} \mathrm{Fe}\left(\mathrm{L}^{\prime}\right)_{3}\right]_{n}$ unit.

Metalloligand strategy is used for production of heterometallic 3D $\mathrm{CPs}$ containing $\mathrm{Cu}(\mathrm{II})$ and trimethyltin as nodes. ${ }^{105}$ The first step of this synthetic way was in situ preparation of generated $2 \mathrm{D} \mathrm{CP}$ with $\left[\mathrm{Cu}(\mu-\mathrm{LH})_{2}\right]_{n}$ unit $\left(\mathrm{LH}_{2}=\right.$ pyridine-2,5-dicarbonic acid), whose reaction with $\mathrm{Me}_{3} \mathrm{SnCl}$ has given heterometallic 3D CP with $\left[\mathrm{Cu}\left(\mathrm{Me}_{3} \mathrm{Sn}\right)_{2}(\mu-\mathrm{L})_{2}\right]_{n}$ unit (Scheme 6). The obtained CP is 4,4-connected polymer with sqc topology, which contains the paddlewheelshaped core consisting of two heterometallic $\mathrm{Sn}(\mathrm{Iv}) / \mathrm{Cu}(\mathrm{II})$ macrocycles.
In another interesting example, $3 \mathrm{~d}-4 \mathrm{~d}$ heterometallic $\mathrm{Co}(\mathrm{III})-$ $\mathrm{Zn}$ (II) and $\mathrm{Co}(\mathrm{III})-\mathrm{Cd}(\mathrm{II})$ CPs are obtained from two $\mathrm{Co}(\mathrm{III})$-based metalloligands containing appended groups of arylcarboxylic acids 5-(picolinamido)-isophthalic acid and 4-[(pyridine-2carbonyl)-amino]benzoic acid in different positions. ${ }^{106}$ Arylcarboxylate groups coordinate secondary metal ions, Zn(II) and $\mathrm{Cd}(\mathrm{II})$, with production of different 3D networks (Fig. 7). It is important that all networks show ordered positions of secondary metal ions and unique, even unprecedented network topologies.

An example of using chelating bpy ligands is porous 3D CP with $\left[\mathrm{La}_{2} \mathrm{Cu}\right]_{n}$ unit based on tetranuclear $\mathrm{La}_{4}$ cluster and $\mathrm{Cu}(\mathrm{I})$ metalloligand, including 6,6'-dimethyl-5,5'-dicarboxy-bpy, where bpy is $2,2^{\prime}$-bipyridine. ${ }^{107}$ In flexible porous heterometallic MOFs of rock-salt-type based on $\left[\mathrm{CoL}_{3}\right]^{3-}$ metalloligand $\left(\mathrm{H}_{2} \mathrm{~L}=4,4^{\prime}\right.$-dicarboxy-bpy) and trivalent lanthanide cations $\mathrm{La}, \mathrm{Ce}, \mathrm{Pr}, \mathrm{Nd}, \mathrm{Sm}, \mathrm{Eu}, \mathrm{Gd}, \mathrm{Tb}$, and Er, six carboxylates in the upper part of each coordination octahedron of $\mathrm{Co}(\mathrm{III})-$ metalloligand are bound with $\mathrm{M}$ cations. ${ }^{108}$ If a MOF contains $\mathrm{M}$ cation smaller than $\mathrm{Nd}(\mathrm{III})$, the MOF is crystallized in cubic spatial group $F m \overline{3} m$, while other MOFs with larger M are crystallized in low-symmetric rhombic spatial group Fddd due to asymmetric 10-coordinated bicapped structure of square antiprism of larger $\mathrm{M}$ cation.

There is an interesting using of macrocyclic metalloligand NiL $\quad\left(\mathrm{H}_{2} \mathrm{~L}=\right.$ 1,4-dihydro-2,3-dioxo-5,6:-9,10:13,14-tribenzo $[1,4,8,11]$ tetraazacyclotetradeca-7,11-diene-7,12-dicarboxylate) for production of CPs with $[\mathrm{Mg}(\mathrm{NiL})]_{n},\left[\mathrm{Zr}_{2}(\mathrm{NiL})_{2}\right]_{n}$ and $\left[\mathrm{Pb}(\mathrm{NiL})_{2}\right]_{n}$ units having infinite one-chained helical structures. ${ }^{109}$ All NiL metalloligands in CPs are located in head-to-tail positions (Fig. 8, left column), in order to form very similar single-twisted helical CPs (Fig. 8, right column).

\subsection{Pillar-layered strategy}

Pillared MOFs are widely studied platforms with numerous applications; they consist of 2D layers, which have ligating sites

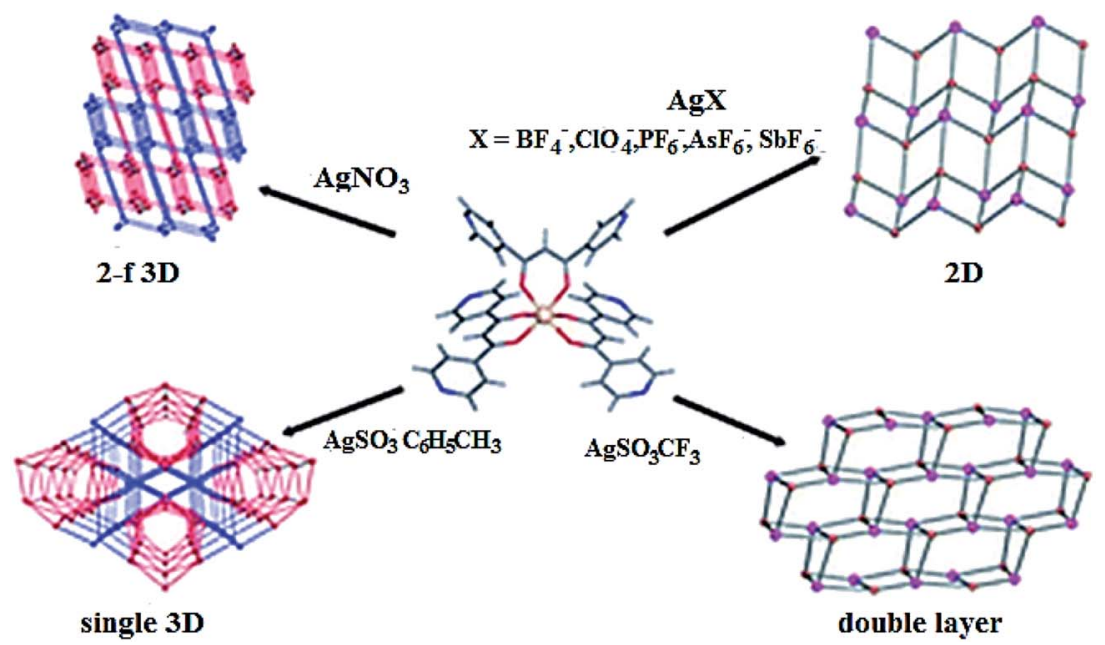

Scheme 5 A dependence of $\mathrm{CP}$ structure on nature of counter-ions during the reactions of $\mathrm{FeL}_{3}$ with $\mathrm{AgX}\left(\mathrm{X}=\mathrm{BF}_{4}{ }^{-}, \mathrm{ClO}_{4}{ }^{-}, \mathrm{PF}_{6}{ }^{-}, \mathrm{AsF}_{6}{ }^{-}, \mathrm{SbF}_{6}{ }^{-}\right.$, $\mathrm{NO}_{3}{ }^{-}, \mathrm{CF}_{3} \mathrm{SO}_{3}{ }^{-}$, tosylate). [Reproduced from ref. 104 with permission from The Royal Society of Chemistry.] 


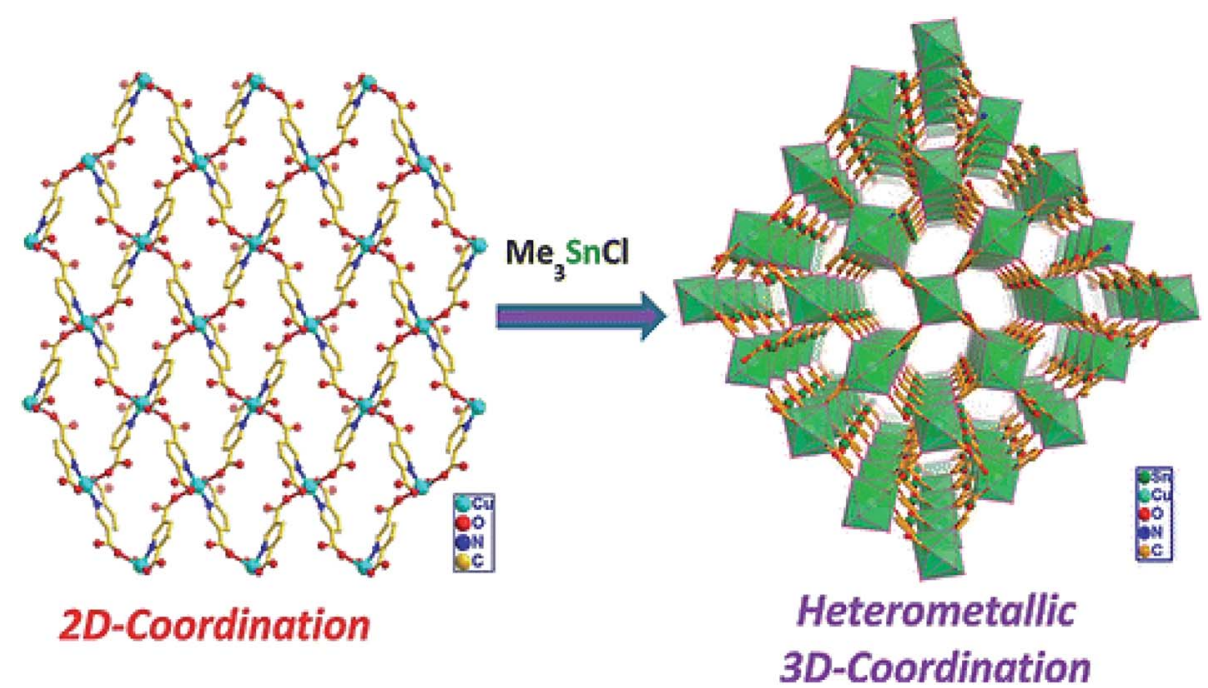

Scheme 6 A metalloligand type of synthetic route for production of heterometallic 3D CP containing Cu(II) and trimethyltin as nodes. [Reprinted with permission from ref. 105. Copyright 2014, American Chemical Society.]

for linking with ditopic columns, as a rule, derivatives of $4,4^{\prime}$ bipy, where $4,4^{\prime}$-bipy is $4,4^{\prime}$-bipyridine, leading to $3 \mathrm{D}$ architectures. Functionalization either with spacers within 2D layers or ditopic columnar ligands allows to easily adjusting structures and properties of pillared MOFs. ${ }^{67}$

Most widely used for building pillared-layered CPs are M-Pp, since they form rigid 2D sheets, which are easily connected by pillared ligands. Thus, for example, Pp paddlewheel frameworks
(PPF family) are obtained using M-Pp and columnar molecules for connection of paddlewheel SBUs. ${ }^{110}$ The PPF assembling can be considered as two-staged process. At the first stage, carboxyl groups of $\mathrm{M}-\mathrm{Pp}$, where $\mathrm{Pp}=$ tetra-(4-carboxyphenyl)-Pp, bind to $\mathrm{M}_{2}(\mathrm{COO})_{4}$ paddlewheel SBUs ( $\left.\mathrm{M}=\mathrm{Zn}, \mathrm{Co}\right)$, which are insensitive to Pp metals. In the resulting $2 \mathrm{D}$ layer consisting of M-Pp and paddlewheel SBUs, axial paddlewheel directions are easily available for connection with ancillary ligands. At the second stage,

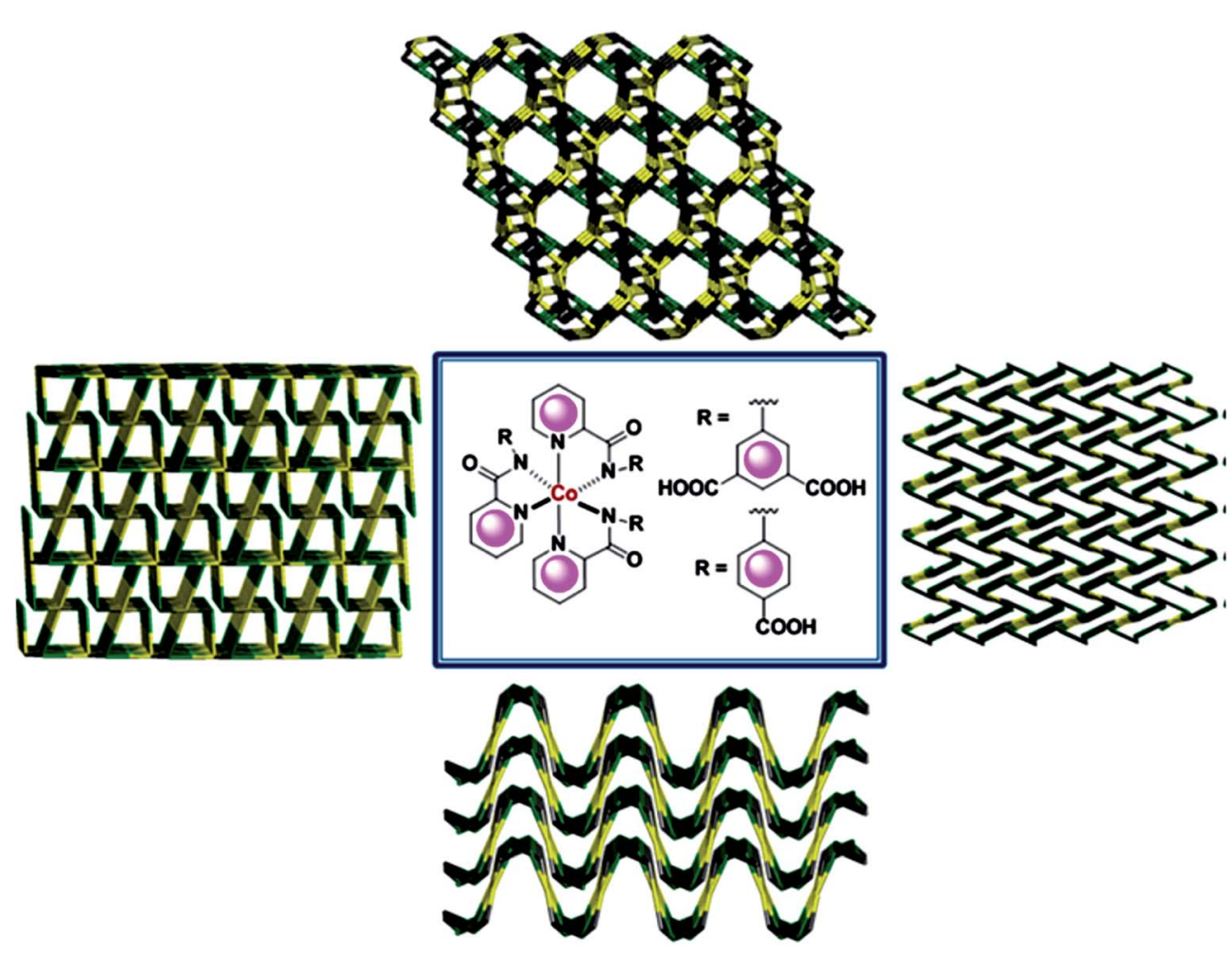

Fig. 7 The synthesis of $\mathrm{Co}(॥ 11)-\mathrm{Zn}(॥)$ and Co(III)-Cd(॥) heterometallic coordination networks. [Reprinted with permission from ref. 106. Copyright 2015, American Chemical Society.] 

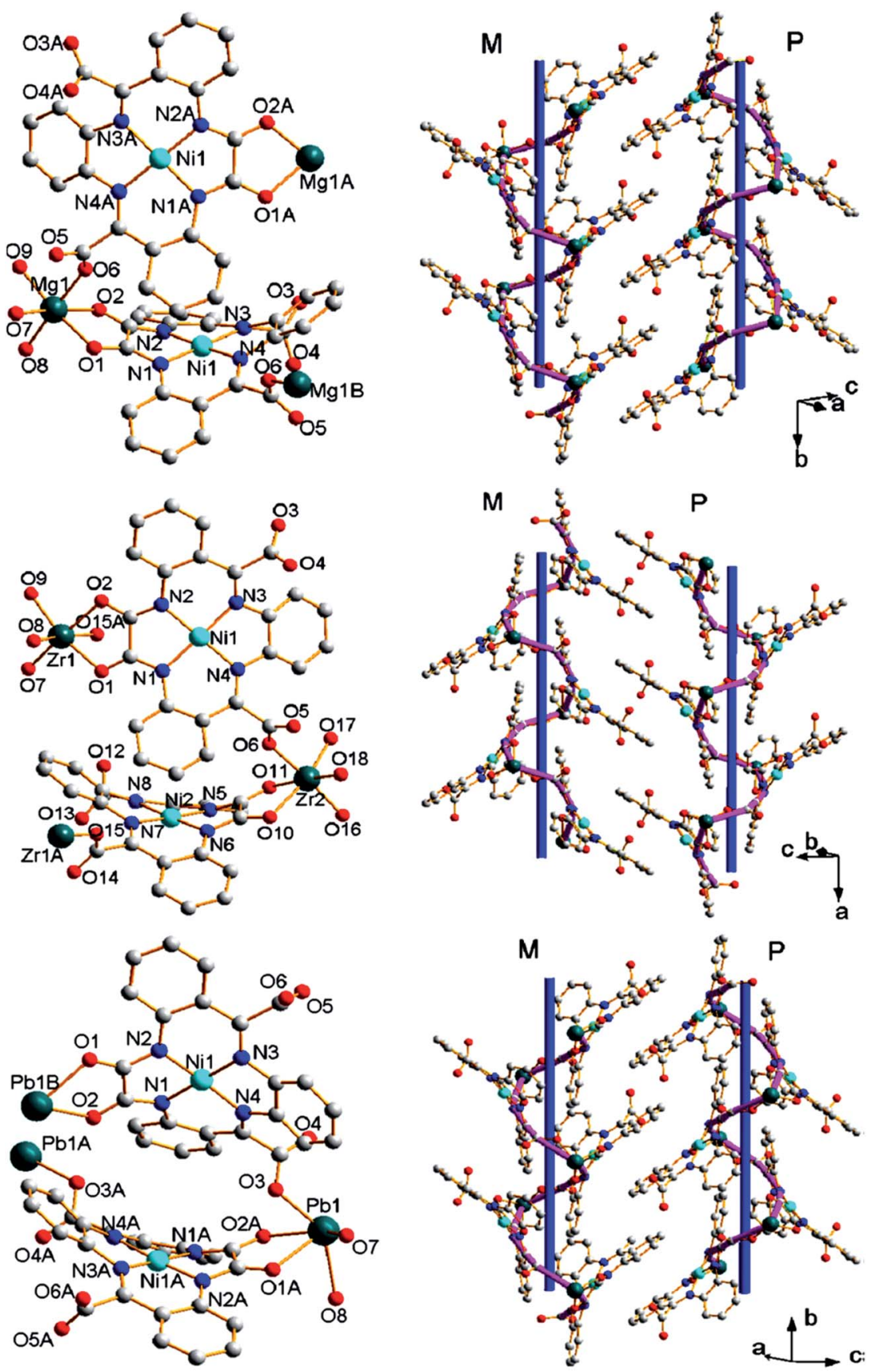

Fig. 8 Coordination environments of metal centers, and metal-binding mode, 《head-to-tail 》) arrangement and saddle shape of NiL (left column) and left- $(\mathrm{M})$ and right-handed $(\mathrm{P})$ helical chains (right column) in [Mg(NiL)] (top), [ $\left.\mathrm{Zr}_{2}(\mathrm{NiL})_{2}\right]$ (middle) and [Pb(NiL) $\left.{ }_{2}\right](\mathrm{bottom}) . \mathrm{Mg}, \mathrm{Zr}$, and $\mathrm{Pb}$, pine green; Ni, cyan; N, blue; O, red; C, grey. [Reprinted from ref. 109 with permission of Springer.]

the class of bipyridyl molecules is used as pillars for linking $2 \mathrm{D}$ layers occupying axial sites. Therefore, for demonstration of targeted M-Pp pillaring, the controlled strategy of production of $\mathrm{Pp}$ based pillared-paddlewheel frameworks PPF-11, including metal pairs $\mathrm{Zn} / \mathrm{Zn}, \mathrm{Co} / \mathrm{Co}, \mathrm{Mn} / \mathrm{Zn}$ and $\mathrm{Fe} / \mathrm{Zn}$, is developed, where first and second metal are the metal centers in a Pp core and in a paddlewheel cluster, respectively, by changing pillaring ligands from 4,4'-bipy to 2,2'-dimethyl-4,4'-bipy. These compounds have $3 \mathrm{D}$ framework, in which $2 \mathrm{D}$ layers are pillared with a steric controlled 2,2'-dimethyl-4,4'-bipy remaining structurally unbound metal centers inside Pp. Therefore, using this approach, a desired control over pillar coordination is reached.

Another example is F-MOF and DA-MOF, which consist of two $\mathrm{Zn}(\mathrm{II})-\mathrm{Pp}$ columns of [5,15-dipyridyl-10,20-bis(pentafluorophenyl)porphyrin]-Zn(II) and [5,15-bis[4-(pyridyl)ethynyl]10,20-diphenylporphinato]-Zn(II), respectively (Scheme 7). ${ }^{\mathbf{9}}$ Photogenerated exciton migrates at a pure distance up to $\sim 45$ Pp pillars during its lifetime in DA-MOF, and only up $\sim 3 \mathrm{Pp}$ pillars in F-MOF with high anisotropy along specific direction. This means that Pp molecular structures play important role in 


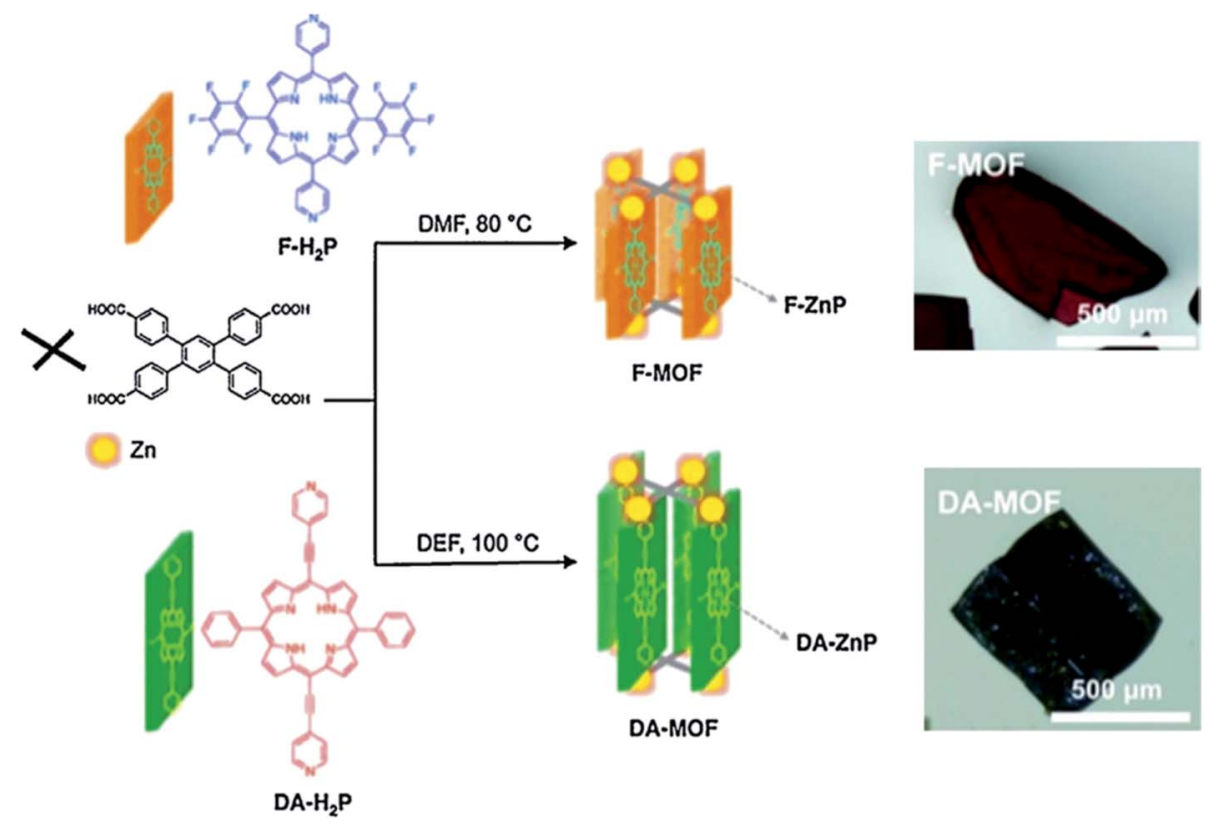

Scheme 7 Synthesis routes of the isostructural F-MOF and DA-MOF compounds and photographs of resulting crystals. [Reprinted with permission from ref. 19. Copyright 2013, American Chemical Society.]

exciton jump. Great distance and directed energy transfer in DA-MOF suggest promising applications of this compound for development of efficient light-harvesting and energytransporting materials.

\subsection{Approach based on supramolecular building blocks}

Other approaches to MOFs design were inspired by success in supramolecular chemistry. Thus, supramolecular building blocks (SBB) approach is a powerful method for achievement of hierarchical assembling, which provide qualitatively far higher level of control over structural properties and porous structures. ${ }^{111}$ This concept is based on using preliminary chosen 0-periodical metal-organic polyhedron (MOP) as a building unit, which allows to self-assembling greater and more complicated ensembles of several metallic nodes and organic spacers at the supramolecular level. As compared with molecular building units, these supramolecular cages or clusters can sharply increment porous architecture, and very often they have well-defined coordination geometry, which results in considerable degree of predictability in synthetic design. MOFs having high symmetry based on highlyconnected polyhedral cage of molecular building units, which actually are SBBs, can provide elegant control over a structure due to their high connectivity, and also can design themselves features of limited nanospace and very high surface area. In most detail, this approach was studied for the example of M-Pp CP by MOP building using $\left.\left[\mathrm{M}_{2} \text { (carboxylate }\right)_{4}\right]$ paddlewheel fragments. It is important that MOPs can be functionalized either on vertices, or on faces.

Using MOPs as building units promotes scale of MOFs to higher complexity level, and can give interesting MOF platforms. Thus, for example, 24-, 18-, or 12-connected MOPs were used as SBBs for formation of highly connected rht-MOFs, ${ }^{112-115}$ gea-MOFs, ${ }^{116}$ and fcu-MOFs, respectively. ${ }^{117}$

Moreover, several isophthalate derived Pp ligands were successfully prepared during building nanometer polyhedral

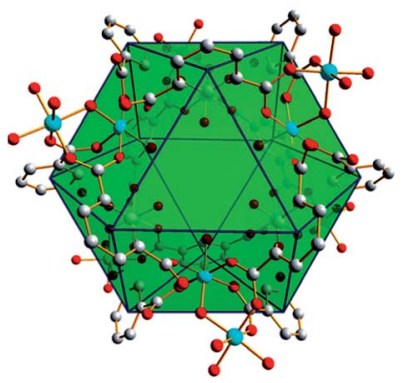

a)

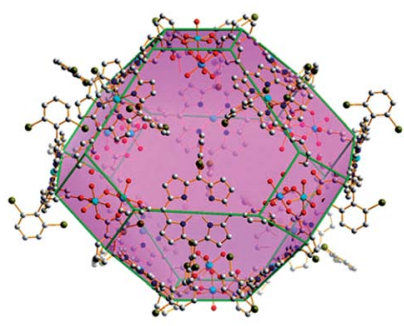

c)

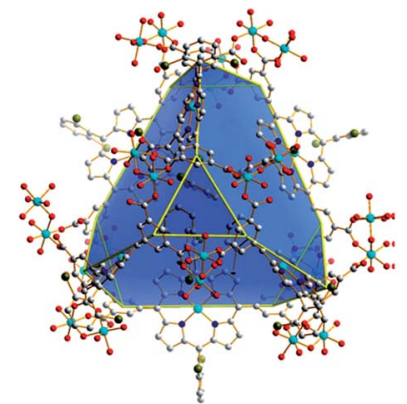

b)

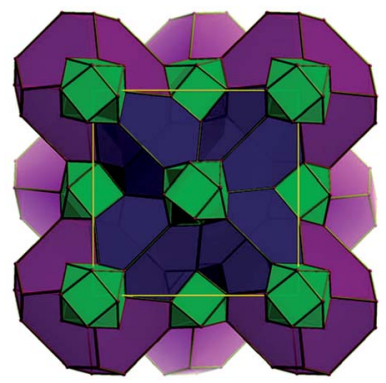

d)
Fig. 9 The three types of polyhedral cages presented in MMPF-3: (a) cubohemioctahedron, (b) truncated tetrahedron, and (c) truncated octahedron. (d) 3D structure of MMPF-3 illustrating how its polyhedral cages are connected. [Reprinted with permission from ref. 61. Copyright 2012, Wiley-VCH Verlag GmbH \& Co. KGaA, Weinheim.] 


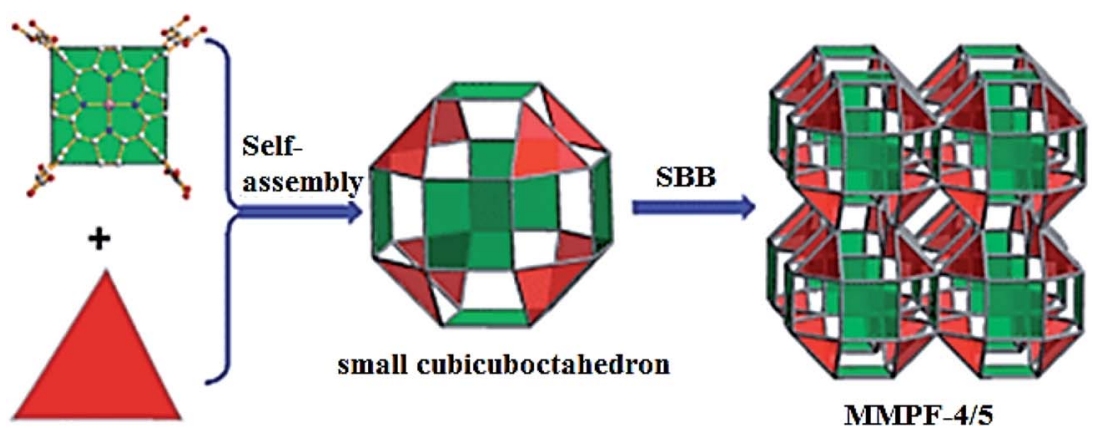

Scheme 8 The scheme of synthesis of MMPF-4 $(M=Z n)$ and MMPF-5 $(M=C d)$ based on small cubicuboctahedral SBBs. [Reproduced from ref. 59 with permission from The Royal Society of Chemistry.]

cage-containing MMPFs. ${ }^{61}$ Thus, MMPF-3 were obtained solvothermally from Pp ligand 5,15-bis(3,5-dicarboxyphenyl)10,20-bis(2,6-dibromophenyl)-Pp and $\mathrm{Co}_{2}\left(\mu_{2}-\mathrm{H}_{2} \mathrm{O}\right)\left(\mathrm{H}_{2} \mathrm{O}\right)_{4}{ }^{-}$ $(\mathrm{COO})_{4}$ molecular building units, which afford cubohemioctahedral SBBs serving as 12-connected nodes in the resulting network of fcu topology. It is worth noticing that MMPF-3 shows three types of polyhedral cage (Fig. 9): a cubohemioctahedron, truncated tetrahedron, and truncated octahedron, which are interlinked.

SBBs are obtained from custom-designed tetra-dicarboxy-Pp self-assembled with $\mathrm{M}$ (II) ( $\mathrm{M}=\mathrm{Zn}$ and $\mathrm{Cd}$ ) cations, which are uniform polyhedrons based on Pp molecular building units. ${ }^{59}$ Faces of $\mathrm{Pp}$ fragments link triangular $\mathrm{M}_{2}\left(\mathrm{CO}_{2}\right)_{3}$ or $\mathrm{M}\left(\mathrm{CO}_{2}\right)_{3}$ fragments with formation of small cubicuboctahedral SBBs, which, in turn, are fused with adjacent SBBs on opposite faces of each Pp fragment. The resulting highly symmetric broadened topology pcu networks, MMPF-4 (M = Zn) and MMPF-5 (M = Cd) show two different polyhedral cages and are permanent microporous (Scheme 8).

\subsection{Approach based on supramolecular building layers}

We shall also notice another conceptual strategy, in particular, the approach based on supramolecular building layers (SBLs), which made it possible to design and produce pillared MOFs with far higher complexity (Fig. 10). ${ }^{118}$ This unique and powerful assembling strategy is based on using pre-targeted 2-periodical MOF layers as SBLs for deliberate building 3-periodical functional MOFs. This is carried out by chemical cross-linking layers through available bridging sites on layers (for example, open metal site or modified positions of organic spacer). SBL structural method requires reasonable choice of organic ligands, which will be pillars for layers. Definitely, this unique strategy is not confined to complicated pillaring; it is also applied to simple pillaring. As a result of countless combinations of crosslinkings, numerous MOFs with certain 3D network topologies can be pre-targeted developed and synthesized using preliminary obtained SBLs. Additional advantage of this approach is the fact that the common framework and topology of the network will remain unchanged basing on pillared layers, which provides almost unlimited expansion of the limited space (for example, cavities and porosity). In addition, it should be noted that if pores or windows of the layers remain unexpanded (i.e. expansion takes place only due to pillars), interpenetration of MOFs is excluded. No less important is the fact that modularity typical of this method provides easy functionalization or introduction of additional functionalities for deliberate applications.

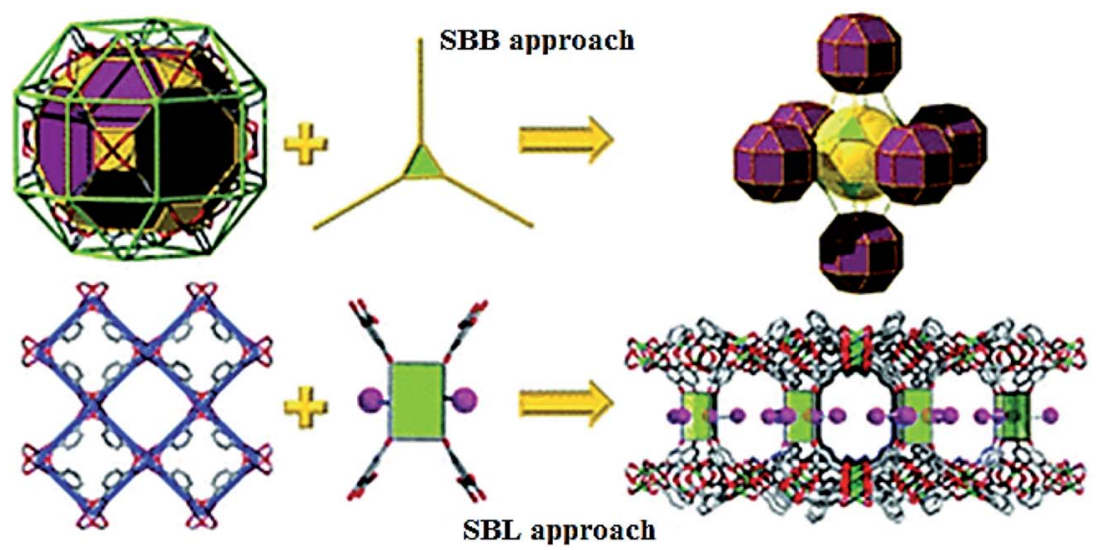

Fig. 10 Two implemented conceptual SBB and SBL approaches facilitating the design and deliberate construction of MOFs. [Reproduced from ref. 118 with permission from The Royal Society of Chemistry.] 
a)

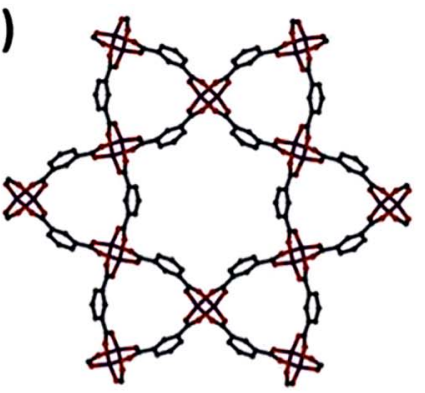

b)<smiles>CCOc1cc(NC(=O)c2cc(NC(=O)c3cccnc3)cc(OC(=O)O)c2)cc(OC(=O)O)c1</smiles>

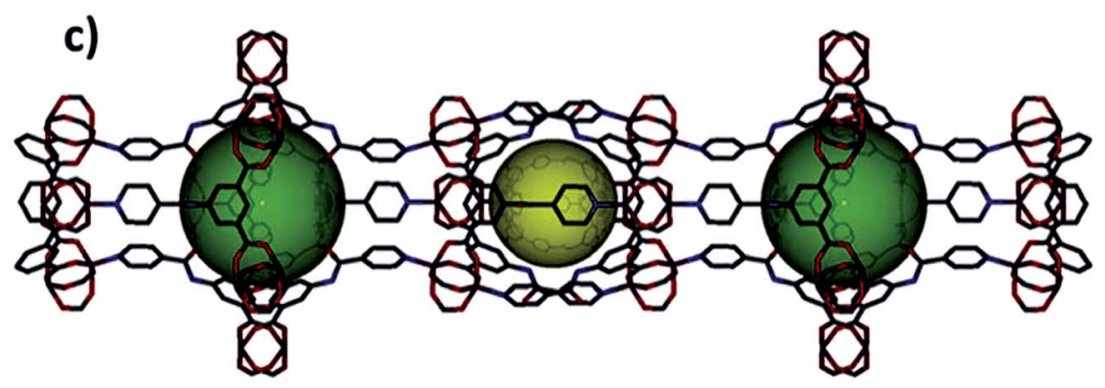

Fig. 11 (a) Layer segment of a kgm-MOF. (b) (Left) 5-(Isonicotinamido)isophthalic acid $\left(\mathrm{H}_{2} \mathrm{~L}^{1}\right)$ and right: 5-(nicotinamido)isophthalic acid ( $\left.\mathrm{H}_{2} \mathrm{~L}^{2}\right)$. (c) Hourglass-shaped channels with two primary types of cavities. C - gray, O - red, N - blue, Cu - plum. [Reproduced from ref. 119 with permission from The Royal Society of Chemistry.]

SBL approach is used for deliberate synthesis of MOF with open massif of amide or amine functionalities inside the porous system. ${ }^{119}$ Two MOF platforms, eea-MOF and rtlMOF based on pillaring kgm-a or sql-a layers with heterofunctional 3-connected organic building units, are directed and built deliberately for desired incorporation and demonstration of amide or amine functionalities (Fig. 11 and 12). Distinctive kgm-a and sql-a networks can be considered as ideal blueprints for targeted 2-periodical MOFs based on assembling square building units produced from well-known metal paddlewheel clusters used as molecular building units. a)

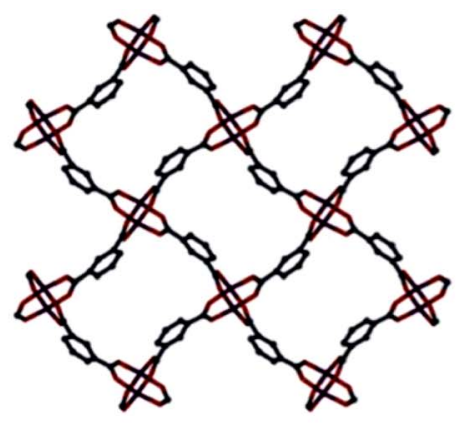

b)

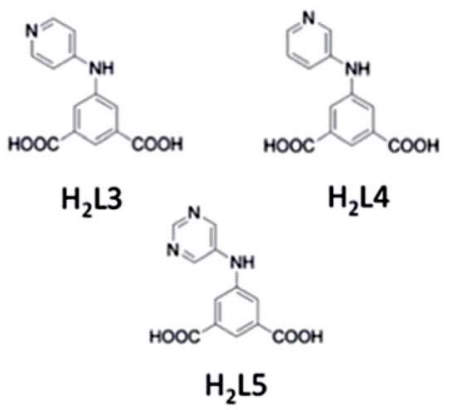

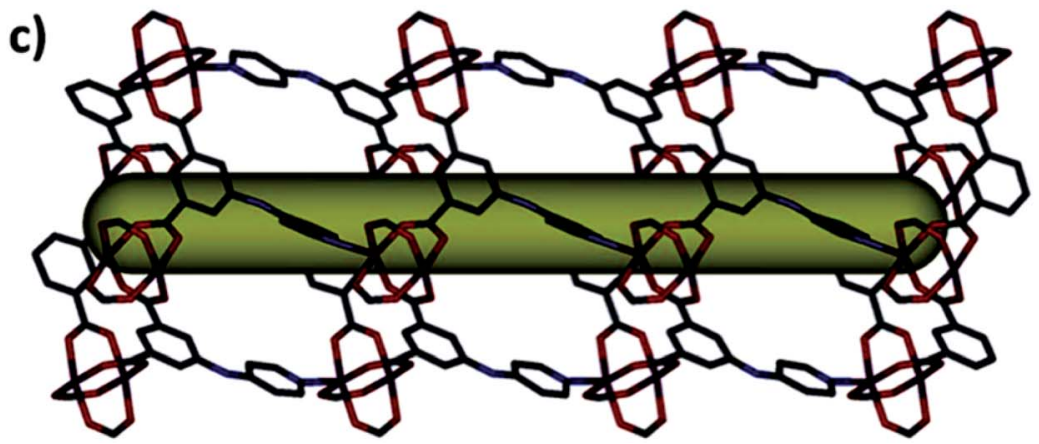

Fig. 12 (a) Layer segment of a sql-MOF. (b) (Left, up) 5-(Pyridin-4-ylamino)isophthalic acid $\left(\mathrm{H}_{2} \mathrm{~L}^{3}\right.$ ). (Right, up) 5-(Pyridin-3-ylamino)isophthalic acid $\left(\mathrm{H}_{2} \mathrm{~L}^{4}\right)$. (Bottom) 5-(Pyrimidin-5-ylamino)isophthalic acid $\left(\mathrm{H}_{2} \mathrm{~L}^{5}\right)$. (c) Projection along the a-axis of the rtl-MOF-2 displaying channel. C gray, $\mathrm{O}$ - red, $\mathrm{N}$ - blue, and $\mathrm{Cu}$ - plum. [Reproduced from ref. 119 with permission from The Royal Society of Chemistry.] 
Other 3-periodical porous MOFs can be potentially targeted through pillaring SBLs based on one of five edge-transitive 2periodical networks: sql (square lattice), kgm (Kagomé), hcb (honeycomb), kgd (Kagomé double), and hex (hexagonal lattice). In addition, the obtained MOF layers can be perceived as SBLs, which are subjected to pillaring through 2-, 3-, 4-, or 6connected organic building units for building desired 3-periodical MOF platforms. ${ }^{118}$

\section{Synthetic methodologies}

\subsection{Conventional synthesis}

By now, quite many approaches to MOF synthesis have been developed, which are usually conventionally divided into two big groups: conventional synthesis and alternative methods of synthesis. ${ }^{\mathbf{1 2 0 - 1 2 3}}$ The term conventional synthesis is applied to reactions performed without heating or using traditional electric heating without parallelization of reactions. Most MOF synthesis is carried out in liquid phase, in which separate solutions of metal salt and ligand are mixed together or a solvent is added to a solid salt and a ligand mixture in a reaction vessel. In order to obtain new MOFs, different factors should be taken into account during synthesis, such as nature of a metal ion, structural features of organic ligand, counterion, and some experimental variables including temperature of reaction, ratio of reacting agents and their concentrations, $\mathrm{pH}$, system of solvents, crystallization methods, etc. ${ }^{\mathbf{1 2 4}}$ Even small variations of only one from several factors having effect on the synthesis process can change dramatically structure of the formed MOFs.

Thus, if reaction between metal(II) acetates and a [1-(2hydroxyphenyl)propylidene]-hydrazide ligand is performed in $1: 2$ (M:L) molar ratio, only $\mathrm{Mn}$ (II) forms $1: 2$ complex in ethanol, while $\mathrm{Cu}$ (II) and $\mathrm{Zn(II)} \mathrm{form} 1: 1$ 1D CPs in methanol (Scheme 9). ${ }^{\mathbf{1 2 5}}$ Interaction of ligand with $\mathrm{Mn}$ (II) acetate in methanol instead of formation of expected Mn(II) complex leads to heterocyclic derivative of quinazoline 2,2-dimethyl-3-[1-(2hydroxy-phenyl)-propylideneamino]-2,2-dimethyl-2,3-dihydro$1 H$-quinazolin-4-one.

Using interaction between 2-substituted 8-hydroxyquinolinate ligand (HL) including Py group and Zn or Cd salts, CPs with $\left[\mathrm{ZnL}_{2}\right]_{n}$ and $[\mathrm{CdL}]_{n}$ units are produced (Scheme 10). ${ }^{\mathbf{1 2 6}}$ The first CP has 2D square network containing meso-helical chains, while the second $\mathrm{CP}$ is a $2 \mathrm{D}$ network made of binuclear $\mathrm{Zn}_{2}$ SBUs. By incorporation of two dicarboxylate anions into the reaction system, four CPs are obtained with $\left[\mathrm{Zn}_{2} \mathrm{~L}_{2}\left(\mathrm{~L}^{\prime}\right)\right]_{n},\left[\mathrm{Zn}_{3^{-}}\right.$ $\left.\mathrm{L}_{2} \mathrm{I}_{2}\left(\mathrm{~L}^{\prime \prime}\right)\right]_{n},\left[\mathrm{Cd}_{2} \mathrm{~L}_{2}\left(\mathrm{~L}^{\prime}\right)\right]_{n}$, and $\left[\mathrm{Cd}_{2} \mathrm{~L}_{2}\left(\mathrm{~L}^{\prime \prime}\right)\right]_{n}$ units $\left(\mathrm{H}_{2} \mathrm{~L}^{\prime}=1,4\right.$-benzenedicarboxylic acid, $\mathrm{H}_{2} \mathrm{~L}^{\prime \prime}=4,4^{\prime}$-biphenyldicarboxylic acid). The L'-based CPs show 2D structures built by cyclic hexamers $\mathrm{Zn}_{6} \mathrm{~L}_{4}$, which are divided in half by coordinated $\mathrm{H}_{2} \mathrm{~L}$. In CPs based on $\mathrm{L}^{\prime \prime}$, four ligands bridge 1D ML chains into 2D layered structure or present an interesting 3D structure in which $\mathrm{L}^{\prime \prime}$ ligands link binuclear $\mathrm{Cd}$ units into multiple meso-helical chains along $a$ and $b$ axes, respectively. Therefore, skeletons of dicarboxylate anions play important role in assembling different MOF structures.

In the case of using organic acid, a base is needed, usually, amine, which is used to deprotonate the acid (future bridging fragment) and to initiate the reaction. At that, it is important to exclude competitive coordination of the base and organic acid.

3.1.1. Solvent evaporation method. A solvent evaporation is most conventional and widely used method of crystal growth which carried out by evaporation or cooling a saturated solution. For this method, the following conditions are needed: crystals grow in saturated solutions, solubility increases as temperature rises, and crystals can appear during cooling,

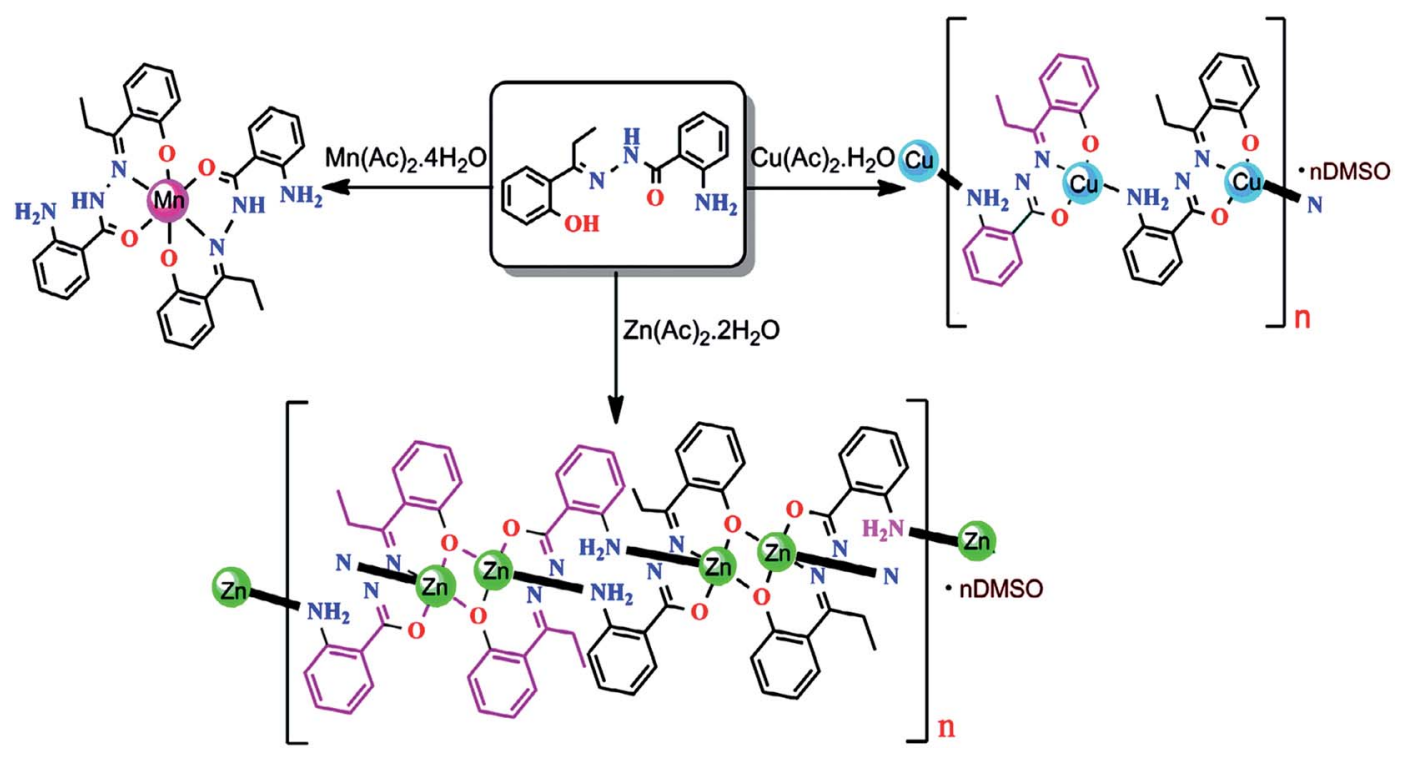

Scheme 9 Versatile coordination behavior of Schiff base ligand, 2-amino-benzoic acid [1-(2-hydroxy-phenyl)-propylidene]-hydrazide, and results of the formation of mono-/polynuclear complexes of $\mathrm{Mn}(॥), \mathrm{Cu}(॥)$ and $\mathrm{Zn}(॥)$. [Reprinted from ref. 125. Copyright 2015, with permission from Elsevier.] 


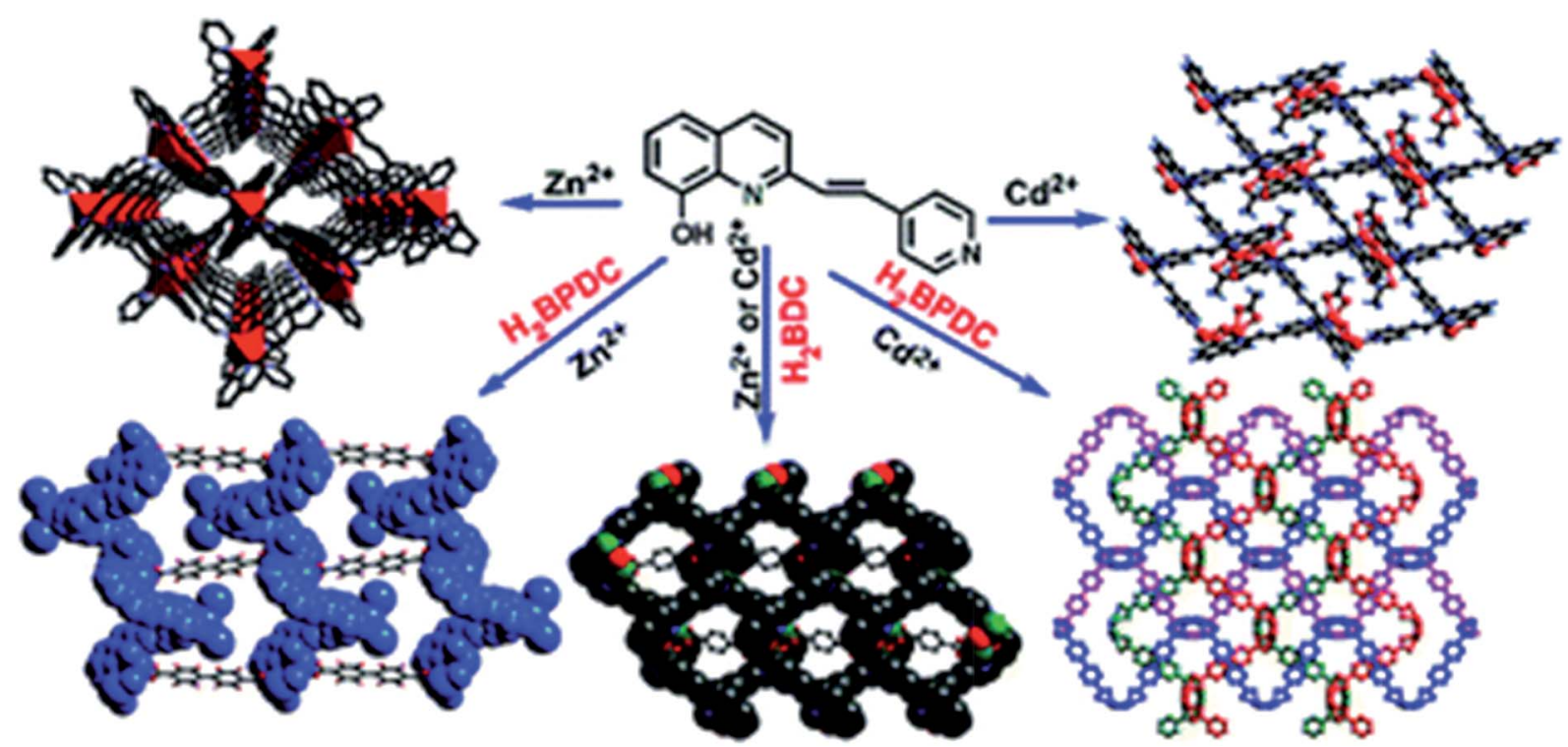

Scheme 10 Synthesis of CPs based on 2-substituted 8-hydroxyquinolinate ligand including Py group, where $\mathrm{H}_{2} \mathrm{BDC}$ is 1,4-benzenedicarboxylic acid and $\mathrm{H}_{2} \mathrm{BPDC}$ is 4,4' -biphenyldicarboxylic acid. [Reproduced from ref. 126 with permission from The Royal Society of Chemistry.]

which should be well controlled, since the cooling rate and final temperature are interrelated. Its main drawback is long duration of the process as compared to other known ordinary methods.

As an example of a simple water-based method of solutionslow evaporation, we shall consider synthesis of $\mathrm{Pb}$-based $\mathrm{CP}$ with $\left[\mathrm{Pb}_{2} \mathrm{Cl}_{2}(\mathrm{HL})_{2}\right]_{n}$ unit, where $\mathrm{H}_{2} \mathrm{~L}=$ pyrazine-2,3-dicarboxylic acid, under optimized conditions. ${ }^{127}$ In the same way, the CP with $\left[\mathrm{Pb}_{3} \mathrm{~L}_{3}\right]_{n}$ unit is obtained without any additives. Reactions between $\mathrm{Zr}(\mathrm{Iv})$ salt and Pp-tetracarboxylic acid lead to MOF with two types of open channels presenting MOF distinguished by $(4,8)$-connected sqc network. It is interesting to notice that MOF remains unchanged in boiling water and in aqueous solutions with $\mathrm{pH}$ changing from 1 to 11 , surprisingly wide range, which MOF can endure. ${ }^{128} 3 \mathrm{D}$ heterobimetallic $\mathrm{CP}$ with $\left[\mathrm{MnL}_{2}-\right.$ $\left.\mathrm{Hg}(\mathrm{SCN})_{4}\right]_{n}$ asymmetric unit $(\mathrm{L}=2$-benzoylpyridine) is synthesized by slow addition of $\mathrm{NH}_{4} \mathrm{SCN}$ and $\mathrm{Hg}(\mathrm{SCN})_{2}$ aqueous solution to methanol solution of Mn chloride and L followed by mixing at room temperature during 12 h. ${ }^{129}$

The final dimensionality of crystal structure depends on such important factor as $\mathrm{M}-\mathrm{L}$ ratio. It is known that $1: 1$ ratio often leads to 1D structures, and high coefficients, as a rule, advance $\mathrm{CP}$ of higher dimensionality. ${ }^{130}$ Not only covalence has relation to these structures: bridging action of non-covalent interactions such as $\mathrm{H}$-bonds or $\pi-\pi$ stacking can change final structures even with very similar components. This result depends strongly on reactivity of involved types and H-bonded donor/acceptor interactions.

Reactions of alkyl substituted aromatic monocarboxylic acids (HL) with transition metal ions in presence of chelating and bridging $\mathrm{N}, \mathrm{N}-$ donor ligands $\left(\mathrm{L}^{\prime}\right)$ give either discrete or polymer complexes depending on position of a substituent on benzoic acid and ability of ancillary ligand to work as chelating or bridging ligand. ${ }^{131}$ For example, when phen was used as an ancillary ligand, and for carboxylic acid was taken 4-tert-butylbenzoic acid, discrete complexes were deposited. However, for the same ancillary ligand with 2,4,6-trimethylbenzoic acid, 1D helical polymers with $\left[\mathrm{ML}_{2}\left(\mathrm{~L}^{\prime}\right)\right]_{n}$ unit $(\mathrm{M}=\mathrm{Mn}, \mathrm{Co}, \mathrm{Cu}$ and $\mathrm{Zn})$ were obtained. This clearly shows ability of $o, o$-disubstituted benzoic acids to form helical polymers, while $p$-substitution on aryl ring leads to discrete complexes.

Ancillary ligands, such as bpy and phen, are often used with multicarboxylate ligands for building new functional CP architectures with chelated units. ${ }^{132}$ Phen as an ancillary ligand is most preferable since it generates 3D supramolecular interactions, such as aromatic $\pi-\pi$ stacking and $-\mathrm{CH}-\pi$ interaction stacking, which provides stability of supramolecular ensembles. $^{133-135}$ In this connection, reasonable combination of bridging carboxylates with phen-like chelating ligands and metal ions has generated many interesting coordinated architectures. As length of a linear spacer increases, a tendency to interpenetration of 3D CP also increases, and if a structure is already penetrated, a degree of interpenetration, as a rule, increases with length of a spacer ligand.

Counter-ions are present in CP structure, when neutral ligands are used. They can have effect on geometrical environment of a metal ion (more or less coordinating counter-ions), and can control generally CP structure by involving into weak interactions or acting as guest molecules in voids of a crystal architecture. As a typical example, we shall consider assembling tripyridyltriazole ligand 3,4-bis(2-pyridyl)-5-(3-pyridyl)1,2,4triazole (L) with dca anion, where dca is dicyanamide, and different $\mathrm{Cd}(\mathrm{II})$ salts in $\mathrm{CH}_{3} \mathrm{OH}-\mathrm{H}_{2} \mathrm{O}$ medium, which leads to CPs with $\left[\mathrm{Cd}_{2} \mathrm{~L}_{2}(\mathrm{dca})_{4}\left(\mathrm{H}_{2} \mathrm{O}\right)\right]_{n}$, $\left[\mathrm{CdL}(\mathrm{dca})\left(\mathrm{ClO}_{4}\right)\right]_{n}$ and $[\mathrm{CdL}(\mathrm{dca})$ $\mathrm{X}]_{n}\left(\mathrm{X}^{-}=\mathrm{Cl}^{-}\right.$and $\left.\mathrm{Br}^{-}\right)$units. ${ }^{136}$ In the first two CPs, Cd(II) centers are linked by $\mathrm{L}$ spacers forming $\left[\mathrm{Cd}_{2} \mathrm{~L}_{2}\right]$ bimetal units, which are bridged with dca anions, thus leading to 1D motif and $2 \mathrm{D}$ layer, respectively. The latter two CPs reflect 3D isostructural 


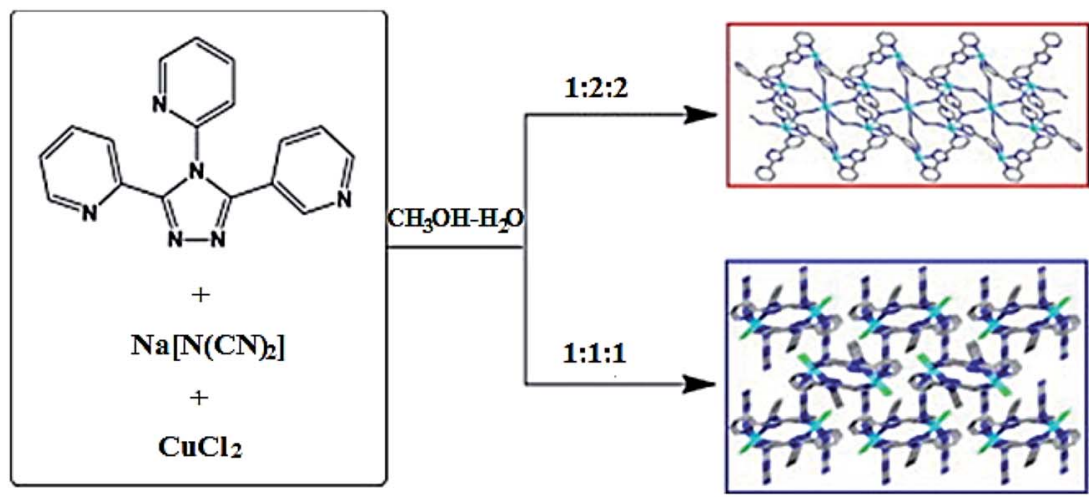

Scheme 11 The assemblies of the tripyridyltriazole ligand 3,4-bis(2-pyridyl)-5-(3-pyridyl)1,2,4-triazole with Cu(॥) chloride and dca anion at different $M-L$ ratios into CPs displaying 1D double-chained massif and 3D chiral dia network, respectively. [Reprinted from ref. 137. Copyright 2014, with permission from Elsevier.]

homochiral coordination networks built by $1 \mathrm{D}[\mathrm{CdL}]_{n}$ helical units and dca bridges. Therefore, Cd(II) salt counter-ions are responsible for different $\mathrm{CP}$ structures. We shall also notice that assemblies of the same ligand with $\mathrm{Cu}(\mathrm{II})$ chloride and dca anion at different $\mathrm{M}-\mathrm{L}$ ratios give $\mathrm{CPs}$ with $\left[\mathrm{Cu}_{5} \mathrm{~L}_{4}(\mathrm{dca})_{10}\right]_{n}$ and $[\mathrm{CuL}(\mathrm{dca}) \mathrm{Cl}]_{n}$ units displaying 1D double-chained massif and 3D chiral dia network, respectively (Scheme 11). ${ }^{137}$

Depending on nature of anion of the initial metal salt $\mathrm{Cu}\left(\mathrm{NO}_{3}\right)_{2}$ or $\mathrm{Cu}\left(\mathrm{ClO}_{4}\right)_{2}$, mixing their aqueous solutions with methanol L solution [L = 3-(2-pyridyl)-4-(4-pyridyl)1,2,4-triazole] leads either to a mononuclear complex $\left[\mathrm{CuL}_{2}\left(\mathrm{H}_{2} \mathrm{O}\right)_{2}\right]\left(\mathrm{NO}_{3}\right)_{2}$, or to $1 \mathrm{D}$ linear $\mathrm{CP}$ with $\left[\mathrm{CuL}_{2}\right]_{n}$ unit, respectively. ${ }^{138}$ It is interesting that incorporation of $\mathrm{NaN}_{3}$ in these assembled systems under similar conditions gives two different $2 \mathrm{D}$ layered networks with $\left[\mathrm{CuL}\left(\mathrm{N}_{3}\right)\right]_{n}$ units, both of which contain double inorganic anions as counter-ions $\left(\mathrm{NO}_{3}{ }^{-} / \mathrm{N}_{3}{ }^{-}\right.$and $\mathrm{ClO}_{4}{ }^{-} / \mathrm{N}_{3}{ }^{-}$, respectively). In addition, $1 \mathrm{D}$ linear massif $\left[\mathrm{CuL}\left(\mathrm{N}_{3}\right)_{2}\right]_{n}$ is obtained with $\mathrm{N}_{3}{ }^{-}$ compensating positive charges with subtle change in reaction conditions. It should be noted that stepwise structural transformations between these crystal phases can also be achieved by direction of azide anion (Scheme 12). In other example, $\mathrm{Cd}(\mathrm{II})$ CPs with $\left[\mathrm{Cd}(\mathrm{L})\left(\mathrm{H}_{2} \mathrm{O}\right) \mathrm{Cl}_{2}\right]_{n},\left[\mathrm{Cd}(\mathrm{L})\left(\mathrm{H}_{2} \mathrm{O}\right) \mathrm{Br}_{2}\right]_{n}$ and $\left[\mathrm{CdLI}_{2}\right]_{2}$ units are assembled from $\mathrm{CdX}_{2}(\mathrm{X}=\mathrm{Cl}, \mathrm{Br}, \mathrm{I})$ and the same ligand. ${ }^{139}$ The first two CPs are isostructural and show 1D loop-like chain, while the third complex has prominent dimeric macrocyclic motif. It is interesting that another $1 \mathrm{D}$ chain $[\mathrm{CdLI}(\mathrm{SCN})]_{n}$ can be reached when $\mathrm{NH}_{4} \mathrm{SCN}$ is incorporated in the assembled system of third complex. Thus, halogenide- and thiocyanateanions in these coordination complexes act not only as counter-ions, but as structure-directing agents.

We shall also notice 3,3'-di(pyrazinamoyl)-bpy $\left(\mathrm{H}_{2} \mathrm{~L}\right)$ ligand consisting of different linking units, which can facilitate CP formation. ${ }^{140}$ Reaction of the ligand with $\mathrm{Cu}$ acetate in presence of $\mathrm{Et}_{3} \mathrm{~N} \cdot \mathrm{HCl}$ gives $\mathrm{CP}$ with $\left[\mathrm{Cu}_{6} \mathrm{~L}_{2} \mathrm{Cl}_{2}(\mathrm{OAc})_{6}\right]_{n}$ unit, at that transconfiguration of bpy-unit presented in the ligand remains in the resulting CP. In other words, a bpy fragment is not chelating,

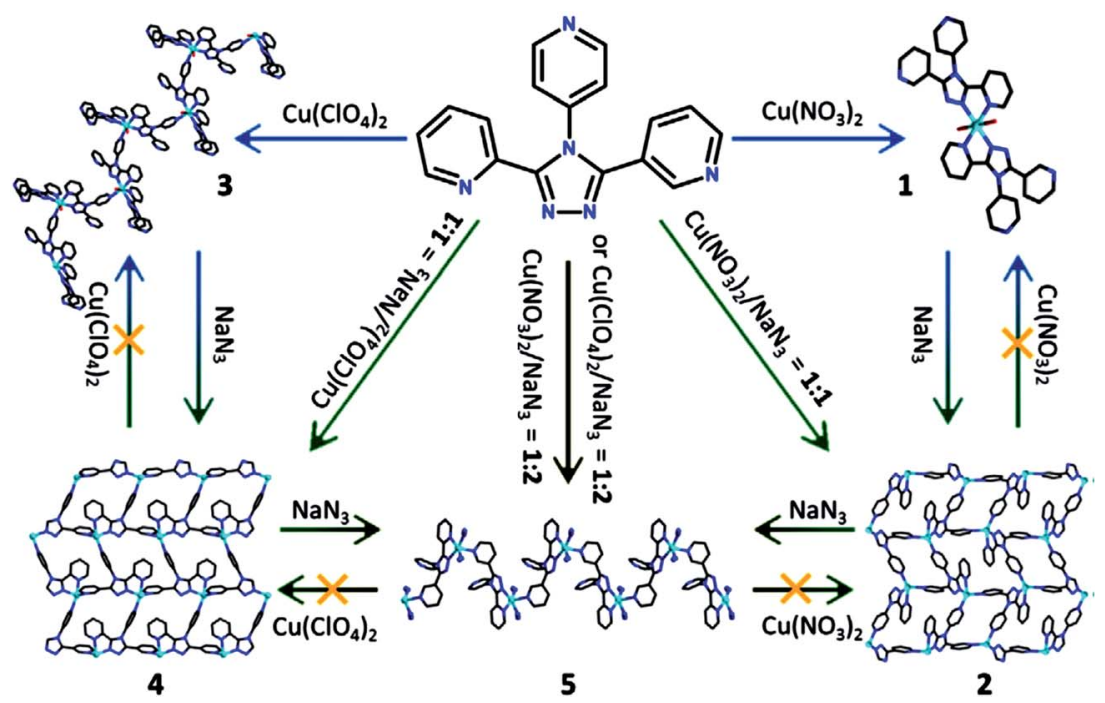

Scheme 12 A diagram illustrating self-assembled and structural transforming processes including 3-(2-pyridyl)-4-(4-pyridyl)1,2,4-triazole, metal salt $\mathrm{Cu}\left(\mathrm{NO}_{3}\right)_{2}$ or $\mathrm{Cu}\left(\mathrm{ClO}_{4}\right)_{2}$, and $\mathrm{NaN}_{3}$. [Reprinted from ref. 138. Copyright 2015, with permission from Elsevier.] 
<smiles>C(=N/c1cccnc1-c1ncccc1/N=C/c1ccccn1)\c1ccccn1</smiles><smiles>[Y][Y]1ccnc(C(=O)Nc2cccnc2-c2ncccc2NC(=O)c2ncccc2[X])c1</smiles>
$\mathrm{L}^{3} \mathrm{H}_{2} \mathrm{X}=\mathrm{N}$

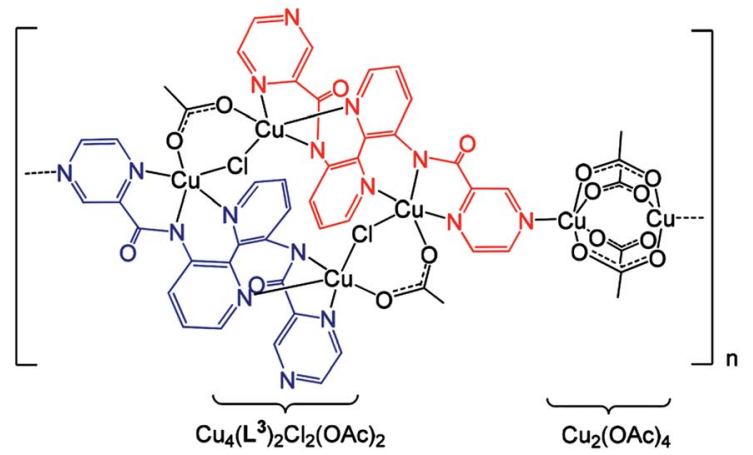

Fig. 13 (Top) Heterocyclic bis-imine and bis-amide ligands prepared from 3,3'-diamino-bpy. (Bottom) The molecular structure of $\mathrm{CP}$ comprised of a $1 \mathrm{D}$ arrangement of $\left[\mathrm{Cu}_{4} \mathrm{~L}_{2} \mathrm{Cl}_{2}(\mathrm{OAc})_{2}\right]$ and paddle wheel $\left[\mathrm{Cu}_{2}(\mathrm{OAc})_{4}\right]$ units; for ease, the two ligands within the $\mathrm{Cu}_{4}$ unit are shown in red and blue. [Reproduced from ref. 140 with permission from The Royal Society of Chemistry.]

and $\mathrm{L}^{2-}$ anion coordinates two $\mathrm{Cu}$ ions by bis-tridentate mode through amide and pyridine nitrogen atoms (Fig. 13). Bimetallic unit is linked to equivalent unit through a pair of 1,3-acetate and $\mu$-chlorine bridges with resulting tetrameric $\left[\mathrm{Cu}_{4} \mathrm{~L}_{2} \mathrm{Cl}_{2}(\mathrm{OAc})_{2}\right]$ core. Coordination of one of pyrazole substituents from each ligand to the third $\mathrm{Cu}$ center of rigid paddlewheel $\left[\mathrm{Cu}_{2}(\mathrm{OAc})_{4}\right]$ unit leads to unique 1D polymer structure.

As the chosen solvents change, the reactions between 2,5thiophenedicarboxylic acid $\left(\mathrm{H}_{2} \mathrm{~L}\right)$ and $\mathrm{Mg}$ nitrate give four different CPs $\mathrm{Mg}_{2} \mathrm{~L}_{2}(\mathrm{Sol})_{2.5} \cdot 0.5 \mathrm{Sol}, \mathrm{MgL}(\mathrm{DMSO}),\left[\mathrm{Mg}_{2} \mathrm{~L}_{2}(\mathrm{Ac})\right]$ and $\left[\mathrm{Mg}_{2} \mathrm{~L}_{2}(\mathrm{DMF})_{2}(\mathrm{EtOH})\left(\mathrm{H}_{2} \mathrm{O}\right)_{2}\right]$ (Sol = ethylene glycol).$^{\mathbf{1 4 0}}$ Coordinated molecules of solvents take different coordination regimes, which play important role in building CP structure. Thus, in the first CP, the Sol molecules as bidentate bridging ligands advance linking MgL layers in 3D framework. DMSO molecules in the second $\mathrm{CP}$ and $\mathrm{Ac}^{-}$anions in the third $\mathrm{CP}$ are coordinated in $\mu_{2}$-way and $\left(\mathrm{k}^{2}-\mathrm{k}^{2}\right)-\mu_{3}$ regime, respectively, leading to infinite chains as SBU. And, finally, in layered 2D structure of the fourth CP, coordination of DMF, EtOH and $\mathrm{H}_{2} \mathrm{O}$ molecules provides induction of the non-centrosymmertical structure.

There is interest in fast, inexpensive, and ecological methods of production of high quality MOFs with properties rarely obtained under normal conditions. ${ }^{\mathbf{1 4 1}}$ The method is based on using organic salts (instead of their homological protonated organic ligands) as sources of anion spacers, so that their solubility and necessary deprotonation stage are achieved substantially in aqueous solution. For example, high quality MOF-74 based on zinc acetate and 2,5-dihydroterephthalic acid usually obtained in organic solvents can also be synthesized in water and at room temperature.

We shall also notice 《green coordination modulation method $\gg$ for CP synthesis using ecological ethanol and alkali additive. ${ }^{142}$ In particular, most variable architectures, including rod crystals, dual-core shelled peanut-like crystals and doublehead dandelion-like crystals, are obtained through changes in parameters of the reaction.

For synthesis of phase-pure MOFs, an easy seed-mediated approach is developed, which allows to skipping the MOF nucleation stage, which generates mixed cores. ${ }^{\mathbf{1 4 3}}$ In addition, using this method, phase-pure MOF isomers with separate porous structures are obtained, thus showing universality of the method.

3.1.2. Diffusion methods. Diffusion method is one of classic methods for synthesis of well-defined crystalline materials. The most often used procedure is liquid-liquid diffusion, also known as free interface diffusion, in which two or more solutions containing solved reagents are used. As compared with fast mixing solutions containing metal ions and bridging ligands, which makes it possible to obtain microcrystalline product, slow diffusion leads to formation of crystals with pronounced morphologies.

Of interest is a production of 3D plywood-like Ni(II) hexaazamacrocyclic $\mathrm{CP}$ with $\left[\mathrm{NiL}\left(\mathrm{H}_{2} \mathrm{~L}^{\prime}\right)\right]_{n}$ unit $(\mathrm{L}=1,3,6,9,11,14$ hexaazatricyclo[12.2.1.16,9]octadecane, $\mathrm{H}_{4} \mathrm{~L}^{\prime}=1,2,4,5$-benzenetetracarboxylic acid) using slow diffusion. ${ }^{\mathbf{1 4 4}}$ The CP structure includes $[\mathrm{NiL}]^{2+}$ cation and $\left[\mathrm{H}_{2} \mathrm{~L}^{\prime}\right]^{2-}$ anion in molar ratio $1: 1$, in which $\mathrm{Ni}$ (II) ions are connected through bridging ligand $\left[\mathrm{H}_{2} \mathrm{~L}^{\prime}\right]^{2-}$ based on weak $\mathrm{Ni} \cdots \mathrm{O}$ coordination interactions with 1D chain formation. Alternating cross-like 1D chains are stacked in the plywood structure and connected with each other through H-bonds, giving 3D network (Fig. 14).

There is such interesting synthetic method as vapor diffusion, which includes solution of a metal source and a ligand in a solvent, for example, DMF, and its positioning in an open container surrounded with a volatile base solution, such as

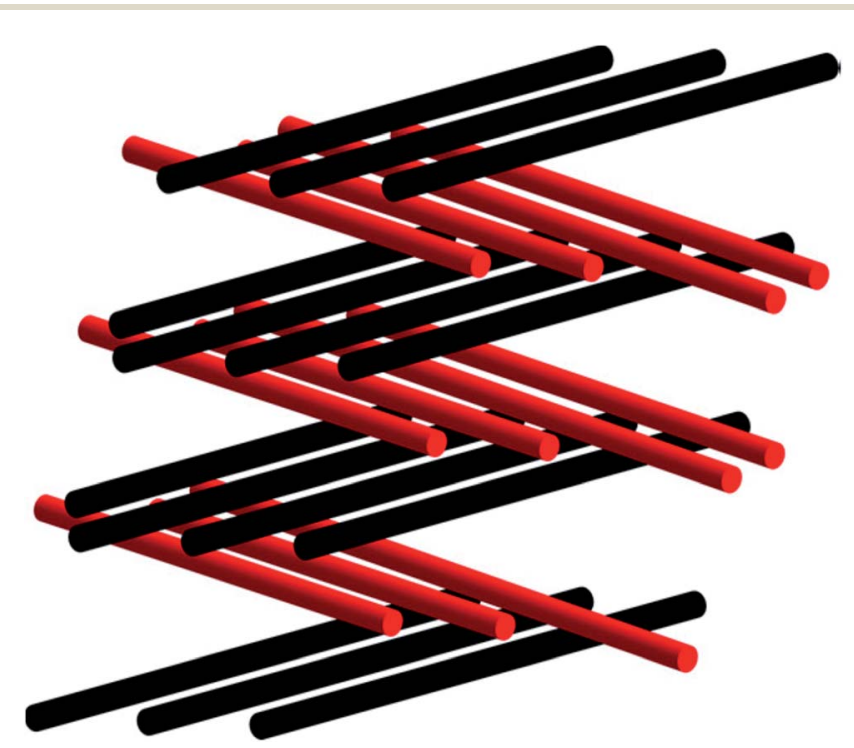

Fig. 14 Representation of cross-like arrangement of $1 D$ chains in $A B A B$ fashion to generate 3D plywood-like array in CP. [Reprinted with permission from ref. 144. Copyright 2016, American Chemical Society.] 
triethylamine. During the synthesis, slow diffusion of a volatile amine into diluted solution of the metal salt and ligand takes place at room temperature and atmospheric pressure, thus increasing the ratio acid ligand: conjugated base through deprotonating, and advancing MOF growth. This strategy makes it possible to form large crystals due to decrease in the reaction time. ${ }^{\mathbf{1 2 0}}$ The diffusion method is more preferable for grow single crystals suitable for $\mathrm{X}$-ray diffraction analysis instead of non-crystalline or polycrystalline products, especially if they are poorly soluble. However, a drawback of diffusion methods is too long time of synthesis (1-2 weeks). In addition, the diffusion method leads to the production of bulk crystals (several hundred microns), which are mainly used for structural studies, but are not good, usually for practical use because of poor mass transfer.

3.1.3. Hydro(solvo)thermal methods. Hydro(solvo) thermal methods are the most efficient way for production of various CPs. Initially, these methods were developed for synthesis of zeolites, but then they were adapted to synthesis of MOFs and occur so efficient that by now the majority of CPs is obtained particularly using these methods. Hydro(solvo) thermal methods are widely applied in synthesis of new CPs, since their products have high purity, good dispersion, are easily controlled, etc. ${ }^{\mathbf{1 2 0 , 1 4 5 - 1 4 8}}$ The hydro(solvo)thermal reactions are associated with using organic or inorganic solvent at high temperature (usually 80-260 ${ }^{\circ} \mathrm{C}$ ) and autogeneous pressure in a closed system (as a rule, teflon-lined autoclaves or glass vessels). ${ }^{\mathbf{1 4}}$ An obvious advantage of hydro(solvo) thermal methods is that they make it possible to alleviate the solubility problems of chelating ligands and improve the reactivity of the reagents during crystallization. Undoubtedly, during the synthesis it is necessary to take into account some parameters of the reaction, including composition of reagents, temperature and pressure, concentration, time of reaction, $\mathrm{pH}$, solubility, etc. ${ }^{\mathbf{1 4 9}}$ Drawbacks of this method are long time of reaction, low percentage yield, necessity in high temperatures and use of toxic solvents (for example, DMF). ${ }^{\mathbf{1 2 0}}$

Among a vast variety of polytopic ligands used in hydro(solvo)thermal MOF synthesis, the leading role, undoubtedly belongs to carboxylate ligands ${ }^{1-4}$ therefore they, first of all, were used in design of CPs with chelated units. Thus, solvothermal reaction of $\mathrm{Co}$ (II) or $\mathrm{Zn}$ (II) salts with dianhydride of 1,2,4,5benzenetetracarboxylic acid $\left(\mathrm{H}_{4} \mathrm{~L}\right)$ leads to formation of 3D CPS with $\left[\mathrm{Co}_{2} \mathrm{~L}\right]_{n}$ and $\left[\mathrm{Zn}_{4} \mathrm{~L}_{2}\right]_{n}$ units. In the first polymer, $1 \mathrm{D} \mathrm{Co}(\mathrm{II})$ carboxylate chains are interconnected by organic fragment of $\mathrm{L}^{4-}$ ligands. In the second polymer, $1 \mathrm{D} \mathrm{ZnL}^{4-}$ ribbons are interconnected by $\left[\mathrm{Zn}_{2}\left(\mathrm{H}_{2} \mathrm{O}\right)_{3}\right]$ building units creating $3 \mathrm{D}$ porous framework with $1 \mathrm{D}$ rectangular channels. ${ }^{150}$ Often in similar synthesis, ancillary ligands such as 1,2-di(4-pyridyl) ethylene $\left(\mathrm{L}^{\prime}\right)$ are also used apart from the main ones. It is with this ligand, $\mathrm{CP}$ with $\left[\mathrm{ZnL}_{0.5}\left(\mathrm{~L}^{\prime}\right)_{0.5}\right]_{n}$ unit is obtained, which has a complicated 3D framework with (3,4)-connected fsh topology. ${ }^{151}$ An interesting example is $3 \mathrm{D} \mathrm{CP}$ with $\left[\mathrm{Cd}_{3}\left(\mathrm{~L}^{\prime}\right)_{2} \mathrm{~L}_{2}\right]_{n}$ unit synthesized based on a main ligand of 1,2,4-benzenetricarboxylic acid $\left(\mathrm{H}_{3} \mathrm{~L}\right)$ and ancillary ligand of 1,4-bis(imidazolyl)butane $\left(\mathrm{L}^{\prime}\right)$ under hydrothermal conditions. The obtained
CP relates to extraordinary examples of trinodal $(4,6)$-connected network with fsc topology. ${ }^{152}$ It is worth paying attention to CP with $[\mathrm{MgL}]_{n}$ unit ( $\mathrm{L}=$ perylene-3,4,9,10-tetracarboxylic acid dianhydride) with special hexagonal tube-like morphology, which is produced by self-assembling by hydrothermal method. ${ }^{153}$ It is interesting that this morphology of $[\mathrm{MgL}]_{n}$ is mostly controlled by Ostwald ripening and self-structuring mechanism, at that using organic solvents as additives, the preparation of CPs of different morphologies, for example, hexagonal rings and snowflakes, and also CP sizes from microto nanometers can be controlled.

Hydrothermal reaction of $\mathrm{La}\left(\mathrm{NO}_{3}\right)_{3}$ with 2-pyrimidinecarbonitrile in water gives $3 \mathrm{D}$ MOF with $\left[\mathrm{La}_{2} \mathrm{~L}_{3}\left(\mathrm{H}_{2} \mathrm{~L}\right)\right]_{n}$ unit ( $\mathrm{L}=$ oxalate), which has channels with trapped water molecules. ${ }^{\mathbf{1 5 4}}$ It is important that CP is built from La atoms linking with bis-chelating oxalate ligands.

La-based CPs microplates are prepared using a simple 〈〈bottom-up $\rangle$ method by hydrothermal reaction of La(III) and 〈|green ligand $\rangle$ ethylenediaminetetraacetic acid (EDTA). ${ }^{155}$ Under similar conditions, analogous Ce-EDTA CPs microplates are also prepared. It is important that the mechanism of LaEDTA CPs formation follows the Ostwald ripening mechanism, and its morphology can be easily adjusted by variation the amount of EDTA and the total concentration of reagents. In addition, also several types of micro/nanostructures have been obtained, such as nanocluster particles, nanofibers, big or uniform microplates.

MOF with $[\mathrm{CdL}(\text { phen })]_{n}$ unit is hydrothermally synthesized using heterocyclic 2,2'-biquinoline-4,4'-dicarboxylate ligand (L) and $\mathrm{N}$-containing ancillary ligand. ${ }^{156}$ The complex is infinite $2 \mathrm{D}$ framework networks containing aesthetic meso-helix, which are linked with formation of 6-connected 3D supramolecular architecture through aromatic $\pi-\pi$ stacking and $\mathrm{C}-\mathrm{H} \cdots \mathrm{O}-\mathrm{H}-$ bonds interactions.

CPs with $\left[\mathrm{ZnL}\left(\mathrm{L}^{\prime \prime}\right)\right]_{n}, \quad\left[\mathrm{ZnL}\left(\mathrm{L}^{\prime \prime}\right)\right]_{n}, \quad\left[\mathrm{Cd}_{2}\left(\mathrm{~L}^{\prime}\right)\left(\mathrm{L}^{\prime \prime}\right)\right]_{n} \quad$ and $\left[\mathrm{Cd}\left(\mathrm{L}^{\prime}\right)\left(\mathrm{L}^{\prime \prime}\right)\right]_{n}$ units are obtained through interaction under solvothermal conditions between 1,2-phenylenedioxydiacetic acid $\left(\mathrm{H}_{2} \mathrm{~L}\right)$ and hydroquinone-O, $\mathrm{O}^{\prime}$-diacetic acid $\left(\mathrm{H}_{2} \mathrm{~L}^{\prime}\right)$ with $\mathrm{Zn}$ and $\mathrm{Cd}$ nitrates in presence of ancillary ligand 1,1'-(1,4-butanediyl)bis(imidazole) $\left(\mathrm{L}^{\prime \prime}\right) .{ }^{157}$ It should be also noted hydro(solvo)thermal synthesized CPs with $[\mathrm{Cd}(\mathrm{HL})(\text { phen })]_{n}$, $\left\{[\mathrm{Cd}(\mathrm{HL})(\mathrm{bpy})]_{2}\right\}_{n}$ and $\left[\mathrm{Cd}\left(\mathrm{HL}^{\prime}\right)(\mathrm{bpy})\right]_{n}$ units based on 2-methyl$\left(\mathrm{H}_{3} \mathrm{~L}\right)$ and 2-ethyl-1H-imidazole-4,5-dicarboxylic acid $\left(\mathrm{H}_{3} \mathrm{~L}^{\prime}\right) .{ }^{158}$ The CPs are infinite chains, in which $\mathrm{HL}$ and $\mathrm{HL}^{\prime}$ in $\mu_{2}$-regime are linked with $\mathrm{Cd}$ (II) atoms, while phen or bpy ligands chelate the central ions (Fig. 15).

An interesting example is hydro(solvo)thermally synthesized CPs with $\left.\left[\mathrm{Mn}_{2} \mathrm{~L}_{2} \text { (phen) }\right)_{n}\right]_{n},\left[\mathrm{Mn}_{2} \mathrm{~L}_{2} \text { (phen) }\right]_{n}$ and $\left[\mathrm{Mn}_{4} \mathrm{~L}_{4}(\text { phen })_{4}\right]_{n}$ units based on 1,4-naphthalene dicarboxylic acid $\left(\mathrm{H}_{2} \mathrm{~L}\right) \cdot{ }^{159} \mathrm{~L}^{2-}$ ligands take various coordination regimes in different solvents and concentrations, which provides formation of different crystal structures. Thus, first $\mathrm{CP}$ has $\mathrm{Mn}_{2}$ dimers linked with $\mathrm{L}^{2-}$ spacers, and stacked in 2D structures of grid pattern. The next $\mathrm{CP}$ has 3D framework, which is formed by $\mathrm{Mn}_{2}$ dimers and $\mathrm{L}^{2-}$ spacers, and each $\mathrm{MnO}_{4} \mathrm{~N}_{2}$ node of the third CP is linked with other node through $\mathrm{L}^{2-}$ ligands with formation of $2 \mathrm{D}$ structure (Scheme 13). 
a

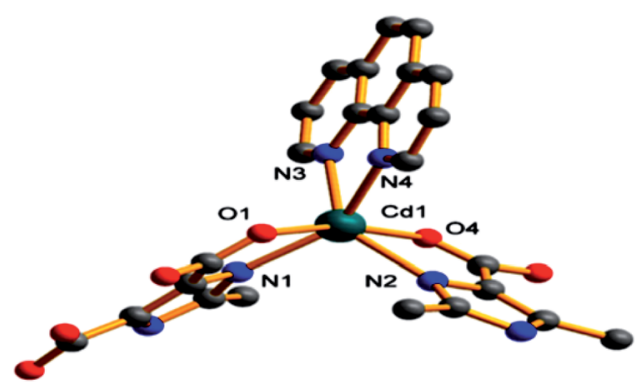

b

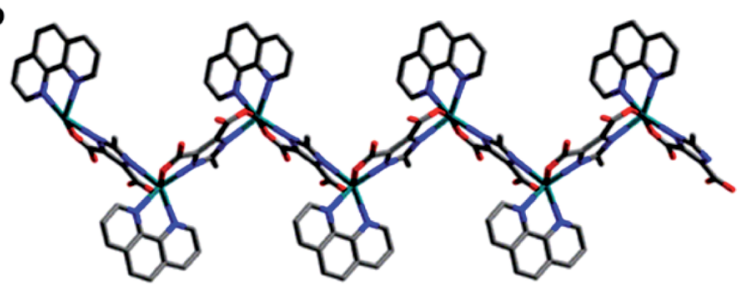

c

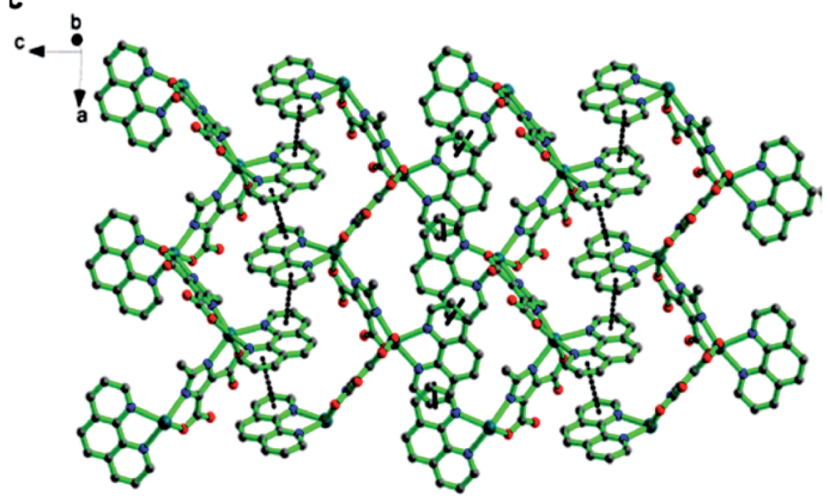

Fig. 15 (a) Molecular square structure of $C P$ with $\left[\mathrm{Cd}(\mathrm{HL})(\text { phen) }]_{n}\right.$ unit based on 2-ethyl-1H-imidazole-4,5-dicarboxylic acid. (b) 1D chain of CP. (c) $2 \mathrm{D}$ sheet of $\mathrm{CP}$ supported by $\pi-\pi$ stacking interactions. [Reprinted from ref. 158 Copyright 2012, with permission from Elsevier.]

It should be also noted synthesized under hydrothermal conditions CPs with $\left[\mathrm{CdL}\left(\mathrm{L}^{\prime}\right)_{0.5}\right](\mathbf{1}),\left[\mathrm{NiL}\left(\mathrm{L}^{\prime}\right)\right](2),\left[\mathrm{Cd}_{2} \mathrm{~L}_{2}\left(\mathrm{~L}^{\prime}\right)_{2}\right](3)$, $\left[\mathrm{CdL}\left(\mathrm{L}^{\prime}\right)\right](\mathbf{4}), \quad\left[\mathrm{NiL}\left(\mathrm{L}^{\prime}\right)\right](5),\left[\mathrm{NiL}\left(\mathrm{L}^{\prime}\right)\right](\mathbf{6}),\left[\mathrm{CdL}\left(\mathrm{L}^{\prime}\right)_{0.5}\right](7)$, [CdL(phen) $](8),\left[\mathrm{Cd}_{3} \mathrm{~L}_{3}\left(\mathrm{H}_{2} \mathrm{O}\right)\right](9)$, and $\left[\mathrm{Cd}_{3} \mathrm{~L}_{3}\left(\mathrm{~L}^{\prime}\right)_{0.5}\right](\mathbf{1 0})$ units including the main ligand 3-carboxy-1-(4'-(2"-carboxy) biphenylmethyl)-2-oxidopyridinium $\left(\mathrm{H}_{2} \mathrm{~L}\right)$ and ancillary $\left(\mathrm{L}^{\prime}\right)$ ligands 1,4-bis(imidazol-1-ylmethyl)benzene (compounds 1 and 2), 2-(6'-(pyridin-2" -yl)-4'-p-tolylpyridin-20-yl)pyridine (compounds 3 and 5), 2-( $4^{\prime}$-( $4^{\prime \prime}$-tert-butylphenyl)-6'-(pyridin- $2^{\prime \prime}$ yl)pyridin-20-yl)pyridine (compounds 4 and 6), 1,10-(1,6-hexanedidyl)bis(imidazole) (compound 7), 1,10-(1,4-butanediyl) bis(imidazole) (compound 10). ${ }^{160}$ It is important that the crystal structures of the obtained CPs are influenced to a great extent by $\mathrm{L}$-anions and $\mathrm{N}$-donor ligands.

Solvothermal reactions of terphenyl-2,5, $2^{\prime}, 5^{\prime}$-tetracarboxylic acid $\left(\mathrm{H}_{4} \mathrm{~L}\right)$ and $\mathrm{M}(\mathrm{II})$ in presence of bpy give three CPs with $\left[\mathrm{ML}_{0.5}(\mathrm{bpy})\right]_{n}(\mathrm{M}=\mathrm{Co}, \mathrm{Ni})$ and $\left[\mathrm{Cu}\left(\mathrm{H}_{2} \mathrm{~L}\right)(\mathrm{bpy})\right]_{n}$ units. ${ }^{161}$

Of interest are systematic arrays of CPs including bpy chelating ligand and different derivatives of isophthalic acid such as 5-(4'-methylphenyl) group (1D linear chain linked with $\mathrm{L}$
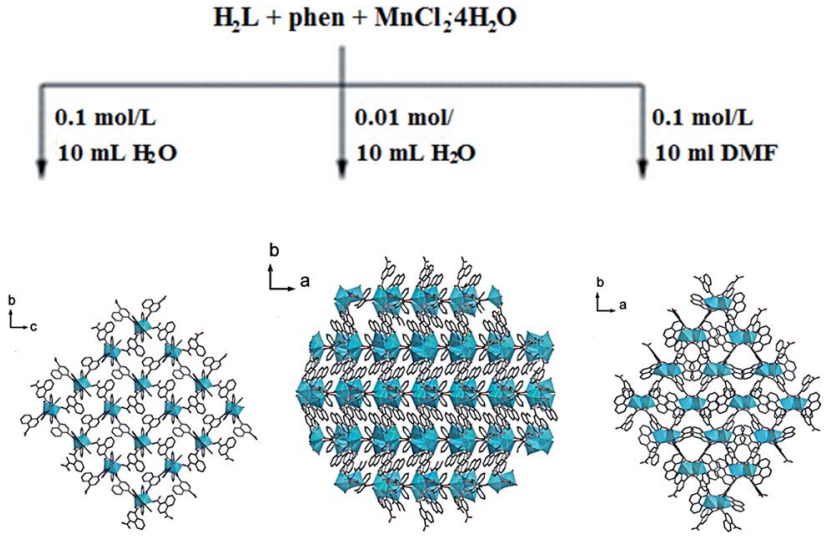

Scheme 13 The formation of various crystal structures in different solvents and concentrations during interaction of 1,4-naphthalene dicarboxylic acid, phen and $\mathrm{MnCl}_{2}$. [Reproduced from ref. 159 with permission from The Royal Society of Chemistry.]

bridge), ${ }^{162} \mathrm{OH}$ group (1D zigzag chains linked by H-bonding and $\pi-\pi$ stacking interactions to form a 3D supramolecular structure) ${ }^{163}$ 5-(4-carboxybenzylamino) group (1D, 2D and 3D frameworks), ${ }^{164}$ 5-(imidazol-1-yl-methyl) group (double-chained and 3D supramolecular structures through $\mathrm{H}-$ bond $/ \pi-\pi$ interactions) ${ }^{165} 4,5-\mathrm{di}\left(3^{\prime}\right.$-carboxylphenyl) group (0D, 1D, 2D and 3D structures) $e t c .{ }^{166}$ Such arrays make it possible to reveal fundamental structure-property relations to define possible directions of practical applications for CPs.

It should be also noted using 3,5-disulfobenzoate (L) in hydrothermal synthesis of Ln-based 2D MOFs with [LnL(phen) $]_{n}$ unit, where $\mathrm{Ln}=\mathrm{La}, \mathrm{Pr}, \mathrm{Nd} .{ }^{167}$ They belong to two $2 \mathrm{D}$ structural types, and their networks have different topologies: the isostructural La and Pr compounds are uninodal 5-connected CP 2-periodical $(6,3)$ networks and the $\mathrm{Nd}$ compound has a binodal 3- and 6-connected kgd network.

It is interesting the using flexible fatty carboxylic acids, for example heptane and hexane diacids, as bridging ligands, and phen derivatives, for example, 2-(3-pyridyl)imidazo[4,5-f]phen and 2-(4-pyridyl)imidazo[4,5-f]phen, as chelating ligands. ${ }^{168}$

Another direction of hydro(solvo)thermal synthesis of CPs with chelated units is linking monomer metal chelates with various bridging ligands. Thus, $\mathrm{CP}$ with $\left[\mathrm{Co}_{2}\left(\mu_{1,1}-\mathrm{N}_{3}\right)_{2}(\mathrm{phen})_{2^{-}}\right.$ $\left.\left(\mathrm{N}_{3}\right)_{2}\right]_{n}$ unit has been obtained by hydrothermal method using a phen as ancillary chelating ligand and bridging azide-linking ligand. ${ }^{130}$ Macrocyclic ML complexes based on 2,3-dioxo5,6,15,15-dibenzo-1,4,8,12-tetraazacyclo-pentadeca-7,13-diene $\left(\mathrm{H}_{2} \mathrm{~L}\right)$ are applied as main building units for preparation of CPs with $\left[\mathrm{Cd}\left(\mathrm{L}^{\prime}\right)(\mathrm{NiL})\right]_{n}$ and $\left[\mathrm{Mn}\left(\mathrm{L}^{\prime \prime}\right)(\mathrm{NiL})_{2}\right]_{n}$ units including bridging ligands 5 -aminoisophthalic acid $\left(\mathrm{H}_{2} \mathrm{~L}^{\prime}\right)$ or 5-aminoisophthalic acid $\left(\mathrm{H}_{2} \mathrm{~L}^{\prime \prime}\right)$. It is important that CPs consist of different 1D chains formed by $\mathrm{L}$ and $\mathrm{L}^{\prime}$ or $\mathrm{L}^{\prime \prime}$ bridge, and they are bound through H-bonds into 2D architecture. ${ }^{169}$

The 2D CPs with $\left\{[\mathrm{ZnL}(\text { phen })]_{2}\right\}_{n}, \quad\left\{[\mathrm{CdL}(\text { phen })]_{2}\right\}_{n}$, $\left\{\left[\mathrm{Zn}\left(\mathrm{L}^{\prime}\right)(\mathrm{phen})\right]_{2}\right\}_{n}$ and $\left[\mathrm{Zn}\left(\mathrm{L}^{\prime}\right)(\mathrm{phen})\right]_{n}$ units are obtained as a result of reaction of $\mathrm{Zn}(\mathrm{II})$ - or $\mathrm{Cd}(\mathrm{II})$-phen with biphenyl-4,4'dicarbonic acid $\left(\mathrm{H}_{2} \mathrm{~L}\right)$ and $4,4^{\prime}$-azodibenzoic acid $\left(\mathrm{H}_{2} \mathrm{~L}^{\prime}\right) .{ }^{170}$ The coordination-induced effect of their morphology is shown; in 
particular, grain and flowery morphology of non-coordinated ligands $\mathrm{H}_{2} \mathrm{~L}$ and $\mathrm{H}_{2} \mathrm{~L}^{\prime}$, respectively, changes with coordination of metal salts. The ridged surface, cracks of $2 \mu \mathrm{m}$ width, parallelepiped structure and feathery appearance are established for the CPs, respectively (Scheme 14).

At the same time, when using bpy-4,4'-dicarboxylate $\left(\mathrm{H}_{2} \mathrm{~L}\right)$ and the respective transition metal(II) salt under hydrothermal conditions, CPs with $\left[\mathrm{MnL}\left(\mathrm{H}_{2} \mathrm{O}\right)_{2}\right]_{n}, \quad\left\{\left[\mathrm{NiL}\left(\mathrm{H}_{2} \mathrm{O}\right)_{2}\right] \cdot 2 \mathrm{H}_{2} \mathrm{O}\right\}_{n}$, $\left[\mathrm{NiL}\left(\mathrm{H}_{2} \mathrm{O}\right)_{2}\right]_{n}$, and $\left[\mathrm{CuL}\left(\mathrm{H}_{2} \mathrm{O}\right)\right]_{n}$ units are obtained, in which each metal ion is coordinated with chelating bpy fragment, two carboxylate oxygen $\mathrm{L}^{2-}$ and water molecules. ${ }^{171}$

There is interest in other $3 \mathrm{~d}-4 \mathrm{f}$ heterometallic CPs with $\left[\mathrm{Ln}_{3} \mathrm{Co}_{2} \mathrm{~L}_{5}(\mathrm{HL})_{5}\left(\mathrm{H}_{2} \mathrm{O}\right)_{5}\right]_{n}(\mathrm{Ln}=\mathrm{Eu}, \mathrm{Gd}, \mathrm{Tb}, \mathrm{Dy}, \mathrm{Ho}, \mathrm{Tm}, \mathrm{Lu})$ unit shaped as 3D sandwich-like framework. ${ }^{172}$

Heterocyclic polycarboxylates are widely used in synthesis of CPs with chelated units. ${ }^{\mathbf{1 - 4}}$ Thus, hydrothermally synthesized are 2D lanthanide CPs with $[\mathrm{LnL}(\mathrm{HL})]_{n}(\mathrm{Ln}=\mathrm{Pr}, \mathrm{Nd}, \mathrm{Sm}, \mathrm{Eu}$ and $\mathrm{Tb}$, $\mathrm{H}_{2} \mathrm{~L}=$ pyridine-2,3-dicarboxylic acid) unit, which are built from sheets consisting of carboxylate-bridging Ln dimers folded along [100] direction and held together by $\mathrm{H}$-bonds between carboxylic groups. ${ }^{173}$ Using 1,4-bis(1H-imidazol-1-yl) butane $\left(\mathrm{L}^{\prime}\right)$ as an ancillary ligand, CPs with $\left[\mathrm{ZnL}\left(\mathrm{L}^{\prime}\right)\right]_{n}$ and $\left[\mathrm{CdL}\left(\mathrm{L}^{\prime}\right)\right]_{n}$ units are obtained, first CP of which shows wave-like layered structure, where layers are additionally stacked through $\pi-\pi$ interactions to generate 3D supramolecular architecture (Fig. 16). ${ }^{174}$ For the second CP, 3D three-fold interpenetrating diamondoid architectures are typical.

It should be also noted the using pyridine-2,5-dicarboxylic, ${ }^{175,176}$ pyridine-2,4-dicarboxylic, ${ }^{177}$ and pyridine-2,6-dicarboxylic $^{\mathbf{1 7 8 , 1 7 9}}$ acids in the design of CPs.
Under hydrothermal conditions from $\mathrm{Co}(\mathrm{OH})_{2}$ and chelidamic acid in the molar ratio $1: 1$, the $\mathrm{Co}(\mathrm{II}) \mathrm{CP}$ with $\left[\mathrm{Co}_{1,5} \mathrm{~L}\right]_{n}$ unit is obtained, which crystallizes into $1 \mathrm{D}$ stair-like structure. ${ }^{\mathbf{1 8 0}}$

There is interest in $\mathrm{CP}$ with $\left[\mathrm{Ca}_{2} \mathrm{~L}\left(\mathrm{~L}^{\prime}\right)\right]_{n}$ unit based on pyrazine-2-carboxylate $(\mathrm{L})$ and benzene-1,3,5-tricarboxylate $\left(\mathrm{L}^{\prime}\right)$ synthesized by hydro(solvo)thermal method forming a double layered network, in which inorganic zigzag chains of coordination $\mathrm{Ca}$ polyhedron are bound with organic ligands. ${ }^{\mathbf{1 8 1}} \mathrm{CPs}$ with $\left[\mathrm{M}_{2} \mathrm{~L}_{4}\right]_{n}$ unit are obtained through interaction between 2,3pyrazinedicarboxylic acid $\left(\mathrm{H}_{2} \mathrm{~L}\right)$ and $\mathrm{Y}$ and $\mathrm{Sm}$ nitrates under hydrothermal conditions in presence of ammonia for $\mathrm{pH}$ regulation. The obtained CPs are isostructural and show a $3 \mathrm{D}$ network structure based on $\left[\mathrm{M}_{2} \mathrm{~L}_{4}\left(\mathrm{H}_{2} \mathrm{O}\right)_{2}\right]$ building units. ${ }^{182}$

Different synthetic strategies are used in hydro(solvo) thermal synthesis of CPs based on the different imidazoledicarboxylic acids: imidazole-4,5-dicarboxylic, ${ }^{183}$ 2-p-methoxyphenyl- $1 H$-imidazole-4,5-dicarboxylic, ${ }^{184}$ 1-(4-carboxybenzyl)$1 H$-imidazole-4,5-dicarboxylic, ${ }^{185}$ and 2-(pyridine-3-yl)- $1 H$-imidazole-4,5-dicarboxylic ${ }^{186}$ acids.

CPs with $\left[\mathrm{PbL}_{2}\right]_{n}$ and $[\mathrm{MnL}]_{n}$ units based on imidazo[4,5-f] phen (L) are synthesized using hydrothermal method. ${ }^{187}$ In the structure of the first polymer, a $\mathrm{Pb}$ (II) center is sixcoordinated and has octahedral geometry. In the second CP, $\mathrm{Mn}$ (II) ion is six-coordinated with formation of distorted octahedral configuration, and the polymer shows 1D supramolecular chain formed via $\mathrm{H}$-bonds.

Hydro(solvo)thermal method proves to be very efficient for production of chiral CPs, which are of great interest not only due to intriguing variety of architectures and topologies, but

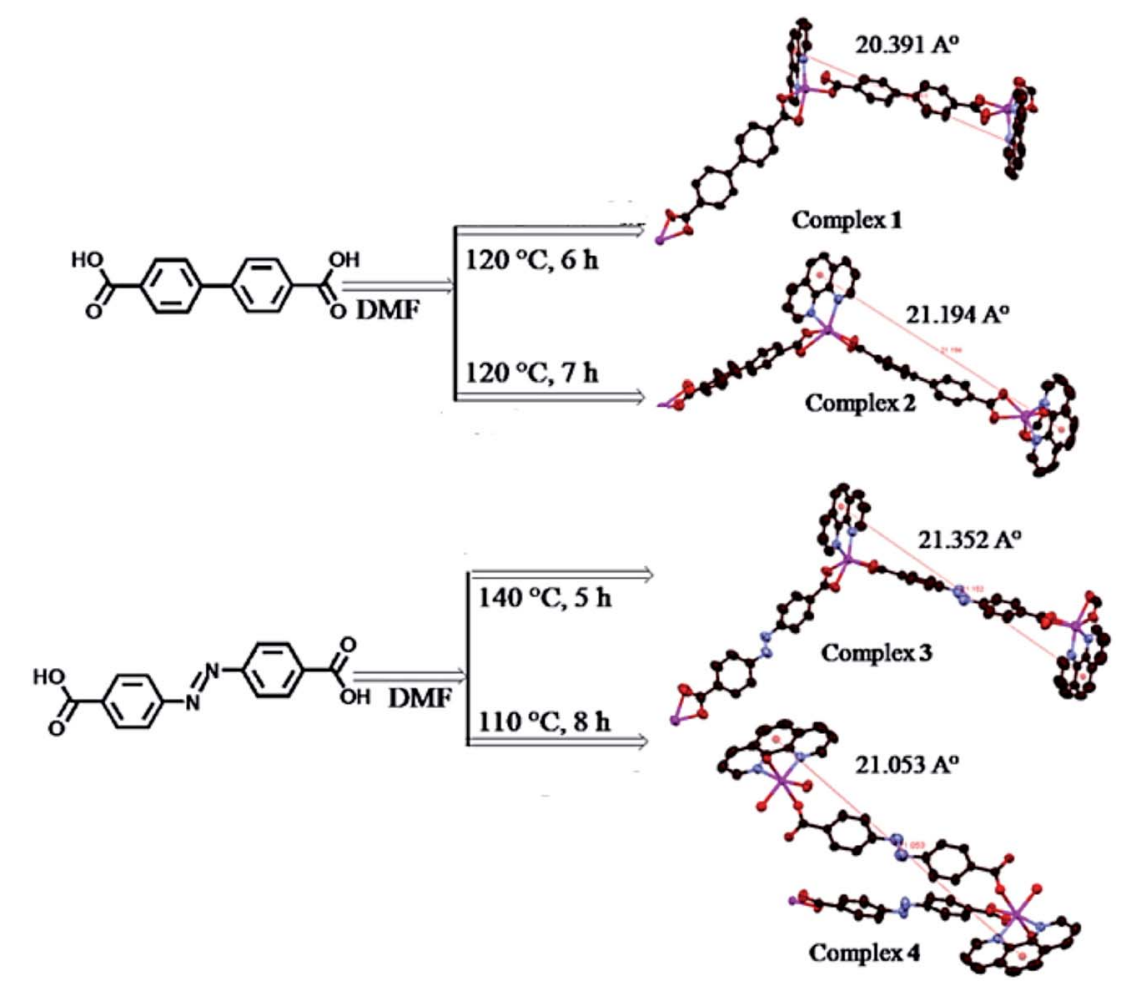

Scheme 14 CPs obtained by the reaction of metal [Zn(I), Cd(I)], phen separately with biphenyl-4,4'-dicarboxylic acid and 4,4'-azodibenzoic acid. [Reprinted with permission from ref. 170. Copyright 2012, American Chemical Society.] 


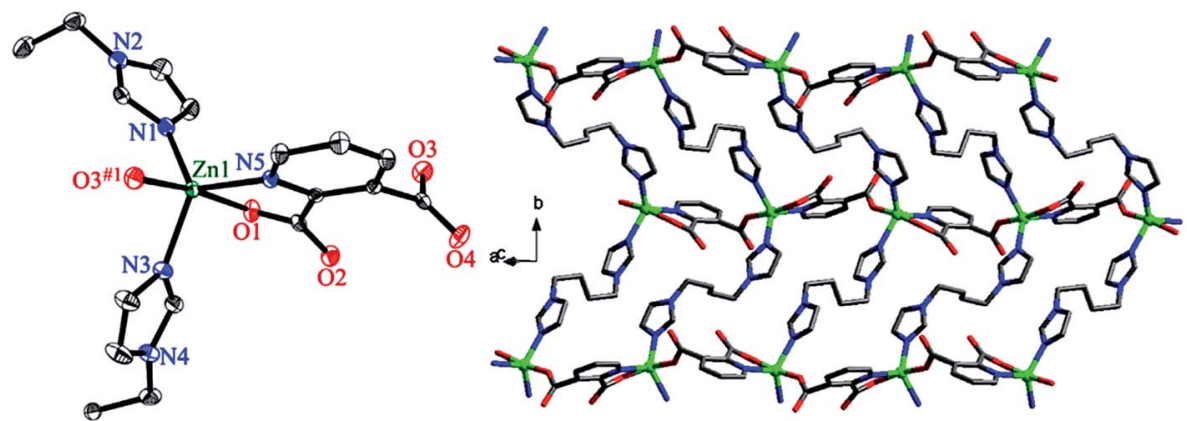

(a)

(b)

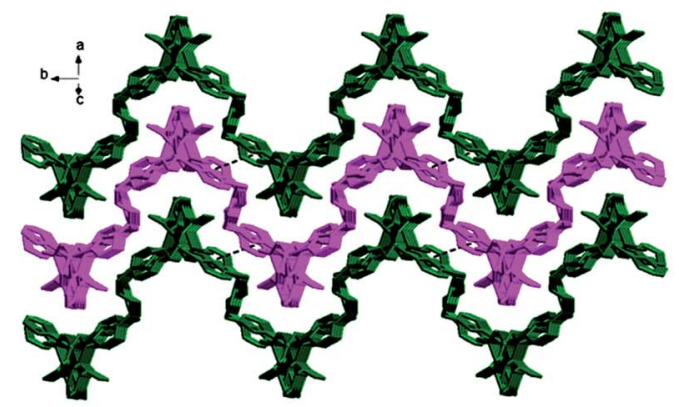

(c)

Fig. 16 (a) Coordination environment of the Zn(॥) atom in CP. (b) View of the 2D undulating layer of CP. (c) View of the 3D supramolecular architecture connected by face-to-face $\pi-\pi$ interactions. [Reprinted from ref. 174. Copyright 2013, with permission from Elsevier.]

because of their potential applications in many areas. ${ }^{92,93,188,189} \mathrm{It}$ is important that chiral CPs can be developed controllably by targeted choice of ligands or metal centers. ${ }^{30,190-193}$ Among the developed by now methods of designing chiral materials, it is worth noticing incorporation of chiral ligands, chiral templates, or chiral ancillary agents, and also spontaneous resolution without chiral auxiliary. ${ }^{194}$ From this view, the most rational method of design of chiral CPs is integration of a chiral center into the structure, such as a chiral ligand; however, this process is not always convenient because of difficulty of a chiral ligand synthesis. ${ }^{195-198}$ In addition, more attention is attracted to use of achiral ligands for building various chiral frameworks, especially by different conformed rotations of a ligand skeleton or self-assembling achiral building units. ${ }^{199}$ Principally important strategy for structuring chiral materials is development of left-hand and right-hand helical structures, which requires efficient transfer of stereochemical information between neighboring chiral helixes. ${ }^{200}$ Considerable contribution to transfer of chirality between helixes can be made using supramolecular interactions including $\mathrm{M}-\mathrm{L}$ bonds and non-covalent interactions, such as $\mathrm{H}$-bonds and $\pi-\pi$ stacking interactions. ${ }^{201}$ Therefore, it is promising to use associated functionalities providing supramolecular interactions and performing efficient chiral transfer along whole supramolecular assembling. At the same time, enantioselective synthesis is straight and efficient method of synthesis of enantiopure chiral-open MOF using enantiopure building units as precursors of reagents. Therefore, many chiral-open frameworks with high thermal stability are obtained using enantioselective synthesis and are successfully used in enantioselective catalysis and separation. It should be noted that chirality is a unique property of CPs with chelated units when tris-chelate is used.

In order to integrate inherent chirality of a ligand and chirality induced by conformation rotations of asymmetric skeleton of the ligand during its coordination, CPs with $\left[\mathrm{CoL}(\mathrm{Py})_{2}\right]_{n},\left[\mathrm{NiL}(\mathrm{Py})_{2}\right]_{n},[\mathrm{CuL}(\mathrm{Py})]_{n},\left[\mathrm{Zn}_{2} \mathrm{~L}_{2}(\mathrm{Py})_{2}\right]_{n}$ and $\left[\mathrm{Cd}_{2} \mathrm{~L}_{2}-\right.$ $\left.(\mathrm{Py})_{2}\right]_{n}$ units are obtained through the reaction between $(1 R, 3 S)$ 1,2,2-trimethyl-3-\{[5-(2-hydroxy)phenyl-1,3,4-oxadiazol-2-yl] $]$ cyclopentanecarboxylic acid $\left(\mathrm{H}_{2} \mathrm{~L}\right)$ and transition metal ions. ${ }^{202}$ Among them, the first two CPs had only right-hand helical chains with homochiral helical discrimination due to formation of interhelical non-classic $\mathrm{H}$-bonds $\mathrm{C}-\mathrm{H} \cdots \mathrm{O}$. Other polymers contained simultaneously alternating right- and left-handed helical chains, interaction between which was due to $\pi-\pi$ interactions ( $\mathrm{CP}$ with $\mathrm{Cu}$ atoms) and interchain bonds $\mathrm{M}-\mathrm{O}-\mathrm{M}$ (CPs with $\mathrm{Zn}$ and $\mathrm{Cd}$ atoms) (Scheme 15). In these compounds, chirality is stipulated by inherent chirality of a ligand and conformation twisting the main asymmetric chain of a ligand. Therefore, the effect of metal ion nature on structure of a chiral $\mathrm{CP}$ is obvious.

Using solvothermal method with Cd(II) salt and enantiopure chiral ligand based on D-isosorbide (L), a homochiral luminescent porous CP with $\left[\mathrm{CdL}\left(\mathrm{H}_{2} \mathrm{O}\right)\right]_{n}$ unit has been synthesized, which is $2 \mathrm{D}$ porous material and forms $1 \mathrm{D}$ channel along $a$ axis with the size $\sim 6.2 \times 4.4 \AA^{2}{ }^{203}$

A chiral CP with $[\mathrm{AgSCN}(\mathrm{phen})]_{n}$ unit obtained using solvothermal reaction between AgSCN and phen shows unusual asymmetric $1 \mathrm{D}$ network formed from a zigzag $[\mathrm{Ag}-\mathrm{S}-\mathrm{Ag}]_{n}$ chain with thiocyanate ions and phen ligands hanging along opposite sides of the chain, respectively (Fig. 17a). ${ }^{204}$ It is important that 


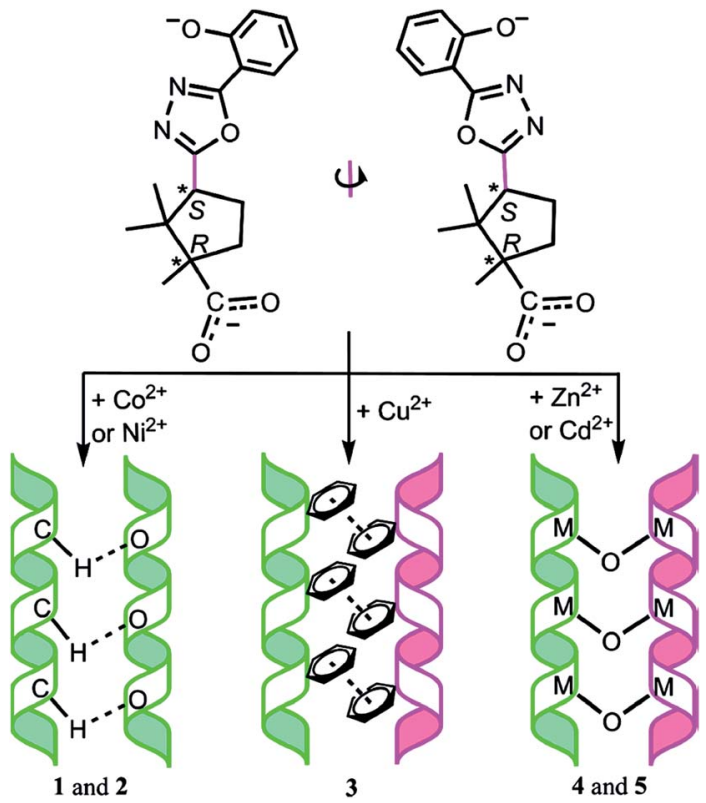

Scheme 15 Conformational twists of the asymmetric skeleton of (1R,3S)-1,2,2-trimethyl-3-\{[5-(2-hydroxy) phenyl-1,3,4-oxadiazol-2yl]\}cyclopentanecarboxylic acid and different interhelical interactions in the prepared CPs. [Reprinted with permission from ref. 202. Copyright 2012, American Chemical Society.]

asymmetry of the chain is clear in $b$ direction (Fig. 17b), and in one unit cell there are two $[\mathrm{AgSCN}(\mathrm{phen})]_{n}$ chains stacked in opposite direction (Fig. 17c). It is interesting to notice that the scheme of the unit cell along $b$ axis is very similar to Chinese "Tai Ji" pattering, which means two opposite principles of nature (Fig. 17d).

Also it is worth noticing homochiral CPs with $\left[\mathrm{ML}\left(\mathrm{H}_{2} \mathrm{~L}^{\prime}\right)\right]_{n}$ unit, where $\mathrm{M}=\mathrm{Zn}$ or $\mathrm{Co}$, based on $\mathrm{D}-(+)$ camphoric acid $(\mathrm{L})$ and 4,4'-methylenebis(3,5-dimethylpyrazole) $\left(\mathrm{H}_{2} \mathrm{~L}^{\prime}\right)$ obtained through interaction between ligands and transition metal ions under hydrothermal conditions. ${ }^{205}$

Of interest is chiral infrequent azolate-based octanuclear metallomacrocyclic 3D $\left[\mathrm{Co}_{2} \mathrm{~L}_{2}\left(\mathrm{H}_{2} \mathrm{O}\right)_{2}\right]_{n}$ complex based on $5^{\prime}$ (pyridin-2-yl)-2H, $4^{\prime} H-3,3^{\prime}$-bi (1,2,4-triazole) $\left(\mathrm{H}_{2} \mathrm{~L}\right)$ (Fig. 18). ${ }^{206}$ It is important that asymmetric polymer unit consists of one-half Co1 and $\mathrm{Co} 3$ ions and one independent $\mathrm{Co} 2$ ion, two $\mathrm{L}^{2-}$ anions, two aqua-ligands, and four and half lattice water molecules, at that each Co(II) ion is six-coordinated and takes slightly distorted octahedral geometry.

Solvothermal synthesis is successful approach to obtaining a broad series of 2D and 3D Pp MOFs. ${ }^{63,207-211}$ For this purpose, most often spacers based on Pp with carboxylic acid and Pylinked functional groups are used, in particular, meso-tetra(4carboxyphenyl)-Pp and meso-tetra(4-pyridyl)-Pp. ${ }^{22,79,82}$ During building MOFs based on Pp spacer, macrocycles can be metallized in situ with the same metal ions, which present in SBUs, ${ }^{58,59,61}$ that is attractive approach for development of high density metallic sites in 3D nanospace, and can be especially enticing for catalysis, if metal centers inside Pp rings, and in SBUs are catalytically active. ${ }^{60,61}$ (a)

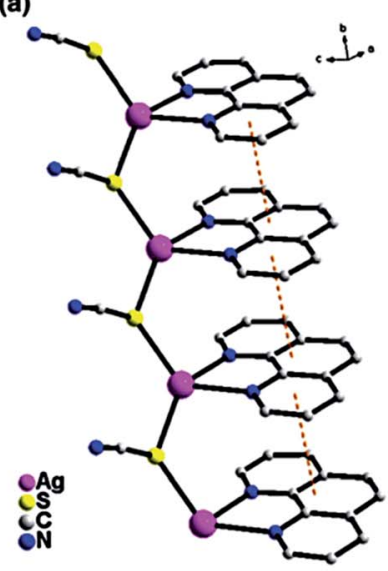

(c)

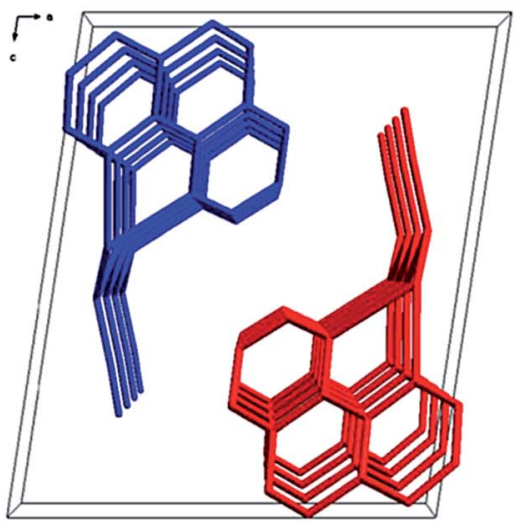

(d)
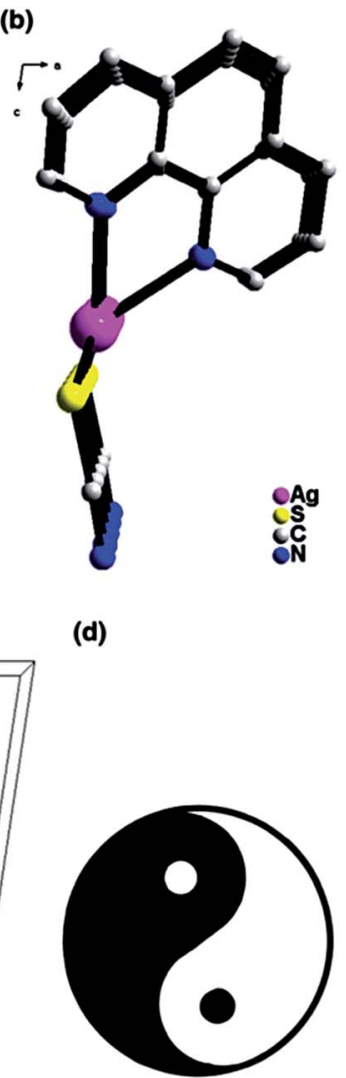

Fig. 17 (a) Diagram of the asymmetric chain in CP exhibiting strong $\pi-$ $\pi$ interactions among the phen rings (orange dash lines). (b) Perspective view of the chain along the $b$ direction. (c) The unit cell packing structure of $C P$ along the $b$ direction. (d) The patterning of 《TTai Ji \. [Reprinted from ref. 204. Copyright 2016, with permission from Springer.]

As a typical example, it should be noted solvothermal reaction of Fe(III) chloride with the tetrakis(4-carboxyphenyl)-Pp in the presence of different bases, which results in the formation of four Fe-Pp MOFs representing three different topologies and inorganic SBU (Scheme 16). ${ }^{212}$ In particular, depending on the synthesis conditions, isolated $\mathrm{Fe}(\mathrm{III})$ octahedra, diiron(II) paddlewheel dimers or extended $\left[\mathrm{Fe}(\mathrm{III})(\mathrm{OH}) \mathrm{O}_{4}\right]_{n}$ chains can be obtained controllably.

Of interest are solvothermally synthesized rare indium-based porous MMPF-7 and MMPF-8 with the pts topology constructed by self-assembly of In(III) and two custom-designed meso-tetra(4carboxyphenyl)-Pp. ${ }^{213}$

Among other Pp ligands for CP synthesis, there is interest in octatopic Pp ligand tetrakis\{3,5-bis[(4-carboxy)phenyl]phenyl\}$\mathrm{Pp}$, which forms $\langle$ pillar-free $\rangle$ highly porous M-Pp framework $\left\{\left[\mathrm{Zn}_{2}\left(\mathrm{H}_{2} \mathrm{O}\right)_{2}\right]_{2} \cdot\left[(\mathrm{ZnL})\left(\mathrm{H}_{2} \mathrm{O}\right)_{2}\right]\right\}_{n}$ called UNLPF-1 in the solvothermal reaction of $\mathrm{L}$ with $\mathrm{Zn}$ nitrate in DMF and acetic acid at $80{ }^{\circ} \mathrm{C}$ during $72 \mathrm{~h} .{ }^{209}$ UNLPF-1 has 3D non-interpenetrating structure with widespread type of SBU, in particular, square paddlewheel $\left[\mathrm{Zn}_{2}(\mathrm{COO})_{4}\left(\mathrm{H}_{2} \mathrm{O}\right)_{2}\right]$, which connects four $\mathrm{L}$ ligands, 


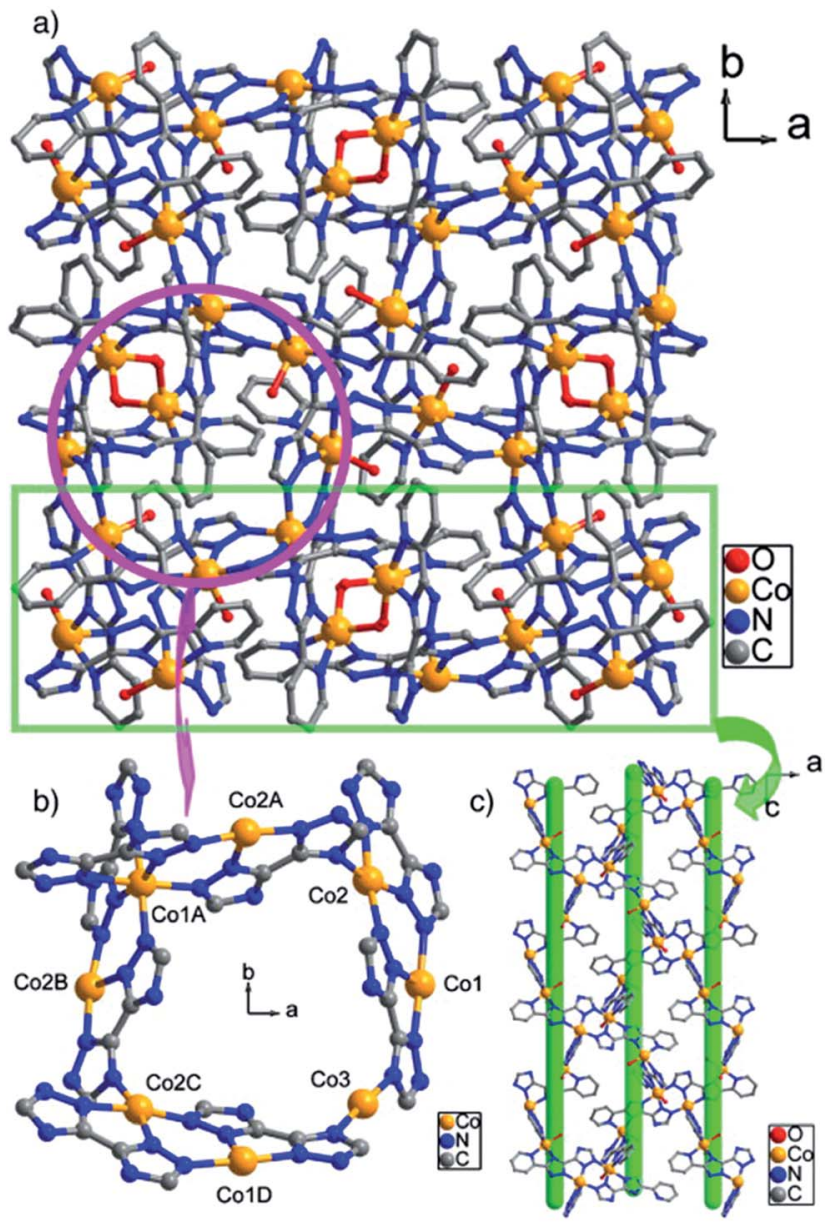

Fig. 18 View of (a) the 3D structure along the $c$ axis, (b) the octanuclear metallamacrocycle, (c) the unidirectional helixes in CP. [Reprinted with permission from ref. 206. Copyright 2013, Elsevier.]

and each L ligand links eight in situ generated (four above and four below Pp plane) SBUs (Fig. 19). It is important that two adjacent Pp macrocycles along with four paddlewheel SBUs form a cavity of great sizes $14.5 \times 23.7 \AA^{2}$. In addition, the same ligand is linked to in situ generated a distorted Co trigonal
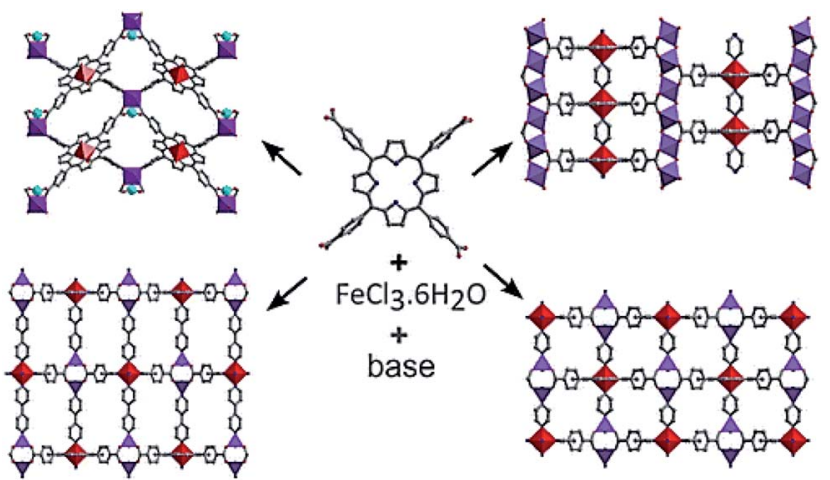

Scheme 16 The CPs obtained by the solvothermal reaction of Fe(III) chloride with tetrakis(4-carboxyphenyl)-Pp in the presence of different bases. [Reprinted with permission from ref. 212. Copyright 2015, American Chemical Society.] prism SBU, producing strong, $(6,8,8)$-connected MMPF-2 with msq topology. ${ }^{58}$

During hydrothermal synthesis of Cd-Pp CPs based on mesotetra-(pyridyl)-Pp, the Pp molecule shows three types of coordination regimes, in which each Pp molecule is linked to one, two, four, and five Cd centers. ${ }^{214}$

Another attractive ligand 5,10,15,20-tetrakis(4,4dipyridylamino-phenylene)-Pp can be coordinated with 4-7 metal centers using its multiple peripheral Py and a Pp core leading to rich structural variety: $\mathrm{H}$-bonding $1 \mathrm{D}$ chains linked by $\left[\left(\mathrm{H}_{2} \mathrm{O}\right)_{2} \mathrm{Cl}_{2}\right]^{2-}$ fragments, 3D structure formed by $\pi-\pi$ stacking interactions between interpenetrating 2D networks, 2D structure with big cavities consisting of 50- and 70-members metallomacrocycles (Fig. 20), complex 2D structure linked with zigzag chains, and stair-like 2D structure containing binuclear $\left[\mathrm{Cd}_{2}\left(\mathrm{CO}_{2}\right)_{4}\right]$ subunits. It is important that 4,4'-dipyridylaminophenylene fragments can rotate around Pp framework, leading to good conformation flexibility of a Pp ligand. ${ }^{215}$

Coordination of $\mathrm{Mn}$ (II) and $\mathrm{Zn}$ (II) acetates with 5,15-bis(4carboxyphenyl)-10,20-dipyridyl-Pp $\left(\mathrm{H}_{4} \mathrm{~L}\right)$ gives two CPs with $[\mathrm{Mn}(\mathrm{HL})]_{n}$ and $\left[\mathrm{Zn}\left(\mathrm{H}_{2} \mathrm{~L}\right)\right]_{n}$ units, respectively. ${ }^{216}$ First $\mathrm{CP}$ has $2 \mathrm{D}$ coordination networks, which are then bound by H-bonds with formation of a 3D network structure. In the second polymer, 1D coordination zigzag chains are obtained, and are then linked by H-bonds into a 2D structure (Scheme 17). It is important that carboxyl residuals in the CPs are noncoordinated and the Py moieties are either coordinated or noncoordinated, at that noncoordinated carboxyl and Py fragments take part in intermolecular H-bonds, which are favorable for formation of $\mathrm{H}$-bonding networks.

There is a great practical interest in using a solvothermal approach for production of uniform thin films based on Zr-Pp

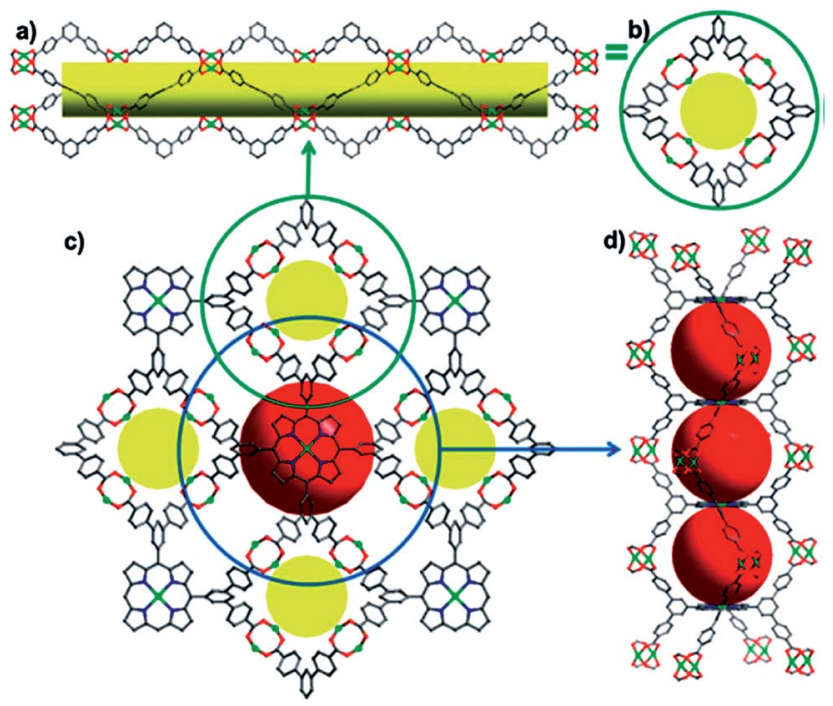

Fig. 19 Side view (a) and top view (b) of a single square-shaped tubular supramolecular building block; (c) 3D network connectivity along the [001] direction between paddlewheels and $V$-shaped terphenyl arms connecting with the Pp spacer; (d) side view of the 1D eclipsed packing of $\mathrm{Pp}$ (cages are represented by red spheres). [Reproduced from ref. 58 with permission from The Royal Society of Chemistry.] 

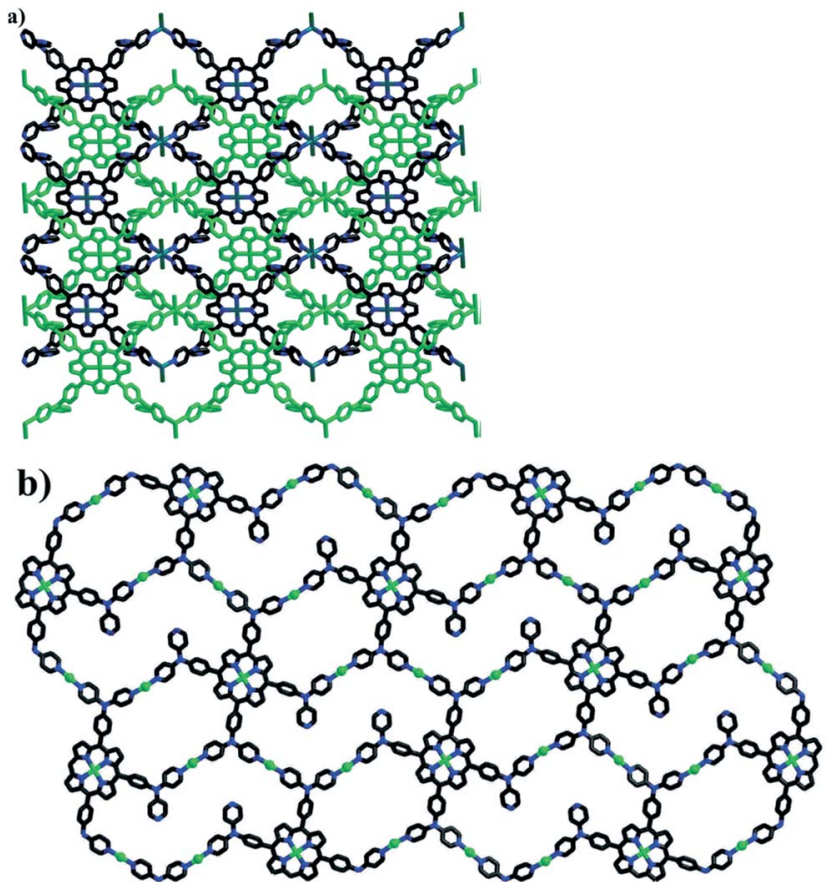

Fig. 20 (a) The interpenetrated 2D coordination networks constructed by $\mathrm{Pp}$ and $\mathrm{MnCl}_{2}$. (b) The 2D network composed of 50- and 70-membered metallomacrocycles, constructed from $\mathrm{Pp}$ and $\mathrm{Cu}(\mathrm{OAc})_{2}$. [Reprinted with permission from ref. 215. Copyright 2013, American Chemical Society.]

MOF (MOF-525) grown on conducting glass substrates. ${ }^{217}$ In particular, the fabricated MOF-525 thin film is electrochemically targeted in aqueous solution and can be used as an amperometric nitrite sensor.

It should be noted the microcrystalline structure containing up to 10 different types of bivalent metals, namely, $\mathrm{Mg}, \mathrm{Ca}, \mathrm{Sr}$, $\mathrm{Ba}, \mathrm{Mn}, \mathrm{Fe}, \mathrm{Co}, \mathrm{Ni}, \mathrm{Zn}$, and $\mathrm{Cd}$, based on MOF-74 with $\left[\mathrm{Zn}_{2} \mathrm{~L}\right]_{n}$ unit $\left(\mathrm{L}=\right.$ dioxidoterephthalate), ${ }^{77,218-222}$ which has high $\mathrm{CO}_{2}$ adsorption capacity under practical atmospheric pressure conditions. The one-pot reaction makes it possible to obtain MOF-74 with 2 (Mg and $\mathrm{Co}$ ), 4 (Mg, Co, Ni, and $\mathrm{Zn}$ ), 6 (Mg, Sr, $\mathrm{Mn}, \mathrm{Co}, \mathrm{Ni}$, and $\mathrm{Zn}$ ), 8 ( $\mathrm{Mg}, \mathrm{Ca}, \mathrm{Sr}, \mathrm{Mn}, \mathrm{Fe}, \mathrm{Co}, \mathrm{Ni}$, and $\mathrm{Zn}$ ), and 10 ( $\mathrm{Mg}, \mathrm{Ca}, \mathrm{Sr}, \mathrm{Ba}, \mathrm{Mn}, \mathrm{Fe}, \mathrm{Co}, \mathrm{Ni}, \mathrm{Zn}$, and Cd) different types of bivalent metals. It turned out that all metal ions used in MOF synthesis are integrated in the same MOF-74 structure, and metal ions are heterogeneously distributed within each of crystalline particles. It should be noted that this approach is also used for incorporation of other metal ions (for example, Ca, $\mathrm{Sr}, \mathrm{Ba}$, and $\mathrm{Cd}$ ), of which the original structure of the MOF cannot be made in the form of monometallic MOF.

Presently combinations of different methods are very popular, and hydro(solvo)thermal method is no exception. ${ }^{223}$ In particular, hydro(solvo)thermal hybrid methods are often applied for synthesis of CPs and functional materials, including nanomaterials. A considerable body of studies has been performed for hybridization of the hydrothermal method with microwaves, electrochemistry, ultrasound, mechanochemistry, optical radiation and hot pressing. Contrary to ordinary heating hydro(solvo)thermal method, which requires a lot of time (as a rule, from half to several days) and high electrical power (more than thousand watts), microwave-assisted heating is a greener approach to synthesis of materials for shorter time (from several minutes to several hours), and lower energy consuming (hundreds watts), as a result of direct and uniform heating a matter. As an example, we can consider UV-absorber $\left(\mathrm{Ti}_{5} \mathrm{O}_{5} \mathrm{~F}_{12}\right) \cdot \mathrm{L}_{2}$ obtained using microwave-heating-assisted hydrothermal synthesis, ${ }^{224}$ whose 3D network is built from $\infty\left(\mathrm{Ti}_{5} \mathrm{O}_{5} \mathrm{~F}_{12}\right)$ infinite inorganic layers separated by guanidinium (L) cations. Under UV irradiation at $254 \mathrm{~nm}$ during $40 \mathrm{~h}$, white microcrystalline powder transformed into light-purple-gray due to reduction of $\mathrm{Ti}$ (IV) to $\mathrm{Ti}(\mathrm{III})$, which is confirmed by magnetic measurements.

3.1.4. Urothermal synthesis. Recently urothermal synthesis has opened a new approach to creation of porous framework materials with promising applications. ${ }^{225-230}$ It is based on using different derivatives of urea as solvents. One of very useful features of urothermal synthesis is reversible coordination of urea derivatives to metal sites, which allows them to competitively link with metal sites of a framework, and in many cases they can be easily removed after crystallization for generation both porosity and open metal sites. Competition for coordination to metal sites among derivatives of urea and other solvents, such as DMF or DEF, is an interesting aspect of this process of synthesis, and can also be used to provide additional structural control.

3.1.5. Ionothermal synthesis. Using room temperature ionic liquids or deep eutectic solvents in CP production is called ionothermal synthesis. ${ }^{231,232}$ Usually in MOFs synthesis ionic

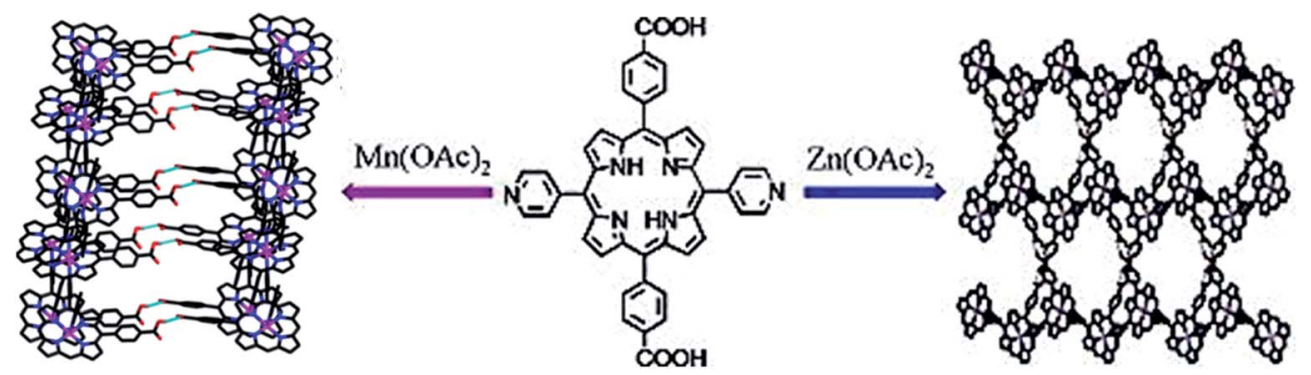

Scheme 17 CPs obtained by the interaction of $\mathrm{Mn}(॥)$ and $\mathrm{Zn}(॥)$ acetates with 5,15-bis(4-carboxyphenyl)-10,20-dipyridyl-Pp. [Reprinted from ref. 216. Copyright 2014, with permission from Elsevier.] 
liquids serve as solvents, reagents, structure-directing agents, charge-compensating templates in anion networks or functional inclusions. Ionic liquids attract attention as a solvent for chemical synthesis due to their unique properties, such as, actually, zero vapor pressure, excellent solvating properties, easy secondary using, and high thermal stability. They received much attention as promising media for design and production of known and unprecedented new MOFs. As compared with water or traditional molecular organic liquid, different and easily tunable properties of the solvent for the ionic liquid supplement considerable attention to eco-friendly and safe solvents. Unfortunately, high cost of ionic liquids limits their usage in large scale synthesis of MOFs.

Most works concerned with ionothermal MOF synthesis is focused on ionic liquids obtained from 1-alkyl-3methylimidazolium. Thus, 3D MOF $\left\{[\mathrm{EMIM}]\left[\operatorname{In}_{3}\left(\mu_{3}-\mathrm{OH}\right)_{2} \mathrm{~L}_{2} \cdot 2 \mathrm{H}_{2}-\right.\right.$ $\mathrm{O}]\}_{n}$ based on 1,2,4,5-benzenetetracarboxylate (L) is synthesized using ionic liquid bromide 1-ethyl-3-methylimidazolium ([EMIM] $\mathrm{Br}$ ) as a solvent. ${ }^{233}$ In this polymer, 7-coordinated pentagonal bipyramidal $\operatorname{In}(1)$ and octahedral $\operatorname{In}(2)$ atoms are linked by $\mu_{3}-\mathrm{OH}$ groups with formation of infinite $\left[\operatorname{In}_{3}\left(\mu_{3}-\mathrm{OH}\right)_{2}\right]_{n}$ inorganic chain along $a$ axis, which are additionally extended by L ligands for creation of $3 \mathrm{D}$ anion microporous framework. The [EMIM] $]^{+}$ cations occupy 1D channels acting as a template and chargecompensating species.

3.1.6. Synthesis in supercritical $\mathrm{CO}_{2}$. Eco-efficient method based on using only supercritical $\mathrm{CO}_{2}$ as a solvent without the addition of any other additive or co-solvent is applied for synthesis of $1 \mathrm{D} \mathrm{Cu(II)}$ MOFs. $^{234}$ For example, neutral $\mathrm{Cu}(\mathrm{acac})_{2}$ chelates and two linear spacers, the bidentate 4,4'-bipy and trimethylene-4, $4^{\prime}$-bipy, were subjected to interaction under compressed $\mathrm{CO}_{2}$ at $60{ }^{\circ} \mathrm{C}$ and $20 \mathrm{MPa}$ during 4 or $24 \mathrm{~h}$, respectively (Fig. 21). The reaction yield of the CPs synthesized through the supercritical route was close to $100 \%$, since both the reagents were almost completely consumed in the performed experiments. It should be noted that success reached in synthesis of different $1 \mathrm{D}$ MOFs is due to high solubility of reagents in supercritical $\mathrm{CO}_{2}$.

3.1.7. Surfactant-thermal method. Another approach to ensure a high degree of control over size and shape of MOF crystals and over their textural properties is use of surfactants (for example, amphiphiles and block-copolymers). ${ }^{235,236}$ It is important that the surfactants play different roles in control over morphology of MOF crystals. In particular, under special conditions, surfactants are self-assembling to form micelles, which can act as nanoreactors. ${ }^{222}$ In addition, in this case, MOFs grow on external surface of a micelle, not inside, so that a surfactant works as a molecular template, not a nanoreactor leading to formation of meso- and/or macro-pores, which together with intrinsic microporosity of MOF leads to particles with hierarchical porosity. ${ }^{237,238}$

As compared with organic solvents, surfactants are more thermally stable and more environmentally. At the same time, surfactants with low or no pressure vapor make reactions in the surfactant medium possible at far higher temperatures. In addition, surfactants have more multifunctional properties than ionic liquids, such as cationic, anionic, neutral, zwitterionic, acidic, basic, etc. Which is more important, their low cost and commercial availability of surfactants make them ideal reaction medium for production of functional materials. Presently, the surfactant medium is used for preparation of nanomaterials, and it should be noted that use of surfactants as reaction medium for the growth of crystalline materials is unprecedented. Janus characteristics of surfactants, which contain hydrophobic and hydrophilic groups, can increase efficiently solubility of metal ions and organic ligands, making them ideal medium for MOF growth.

We note the study of length-controlled synthesis of 1D M-Pp CP using 《 bottom-up》〉 strategy under action of anion surfactant. ${ }^{239}$ For example, using this method, the 1D structure of

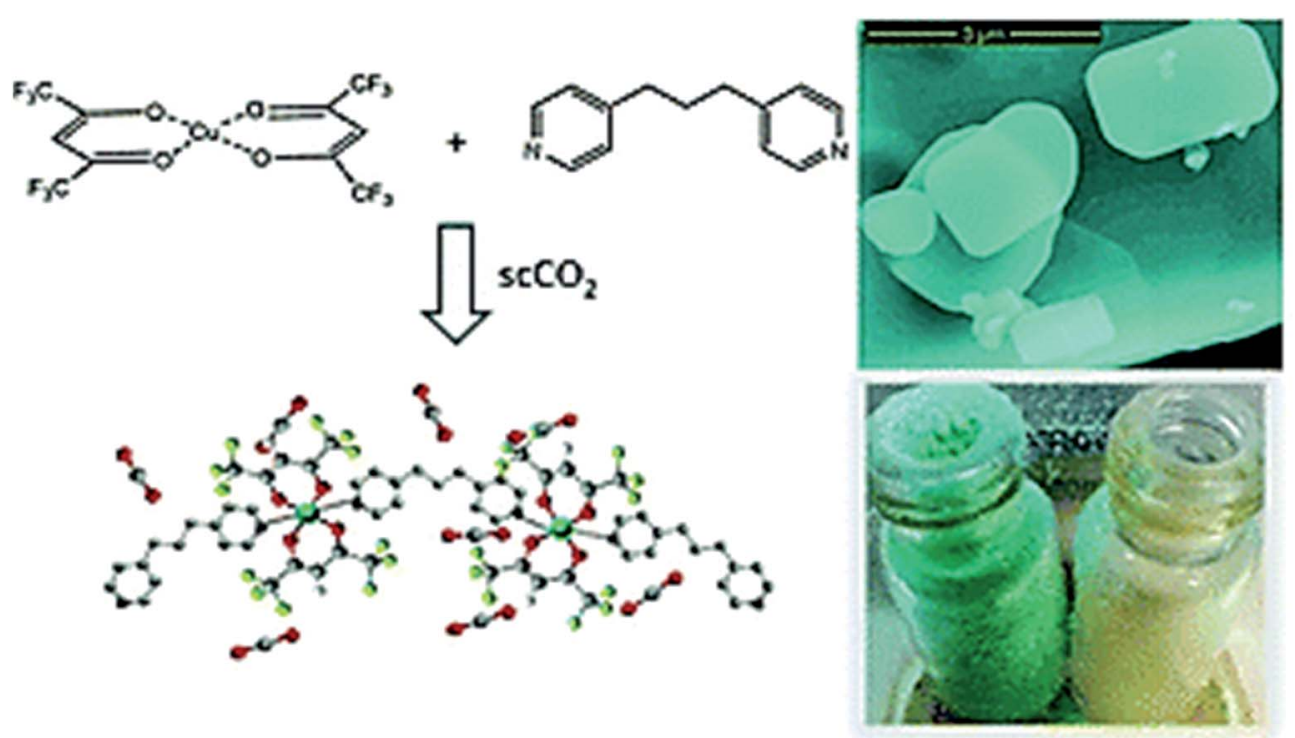

Fig. 21 The preparation of $\mathrm{Cu}(I)$ 1D MOFs using an eco-efficient method based exclusively on supercritical $\mathrm{CO}_{2}$ as a solvent. [Reproduced from ref. 234 with permission from The Royal Society of Chemistry.] 
nanorods and nanowires are obtained. The strategy of growth of crystalline materials is developed: growth of crystalline chalcogenide materials using surfactant-thermal method. ${ }^{\mathbf{2 3 5 , 2 4 0 , 2 4 1}}$ Chiral $\mathrm{Ni}(\mathrm{II})$ mononuclear chelate $\left[\mathrm{NiL}\left(\mathrm{H}_{2} \mathrm{O}\right)_{3}\right]$ and $3 \mathrm{D} \mathrm{CP}$ $\left[\mathrm{NiL}\left(\mathrm{H}_{2} \mathrm{O}\right)\right]_{n}$ based on thiazolidine 2,4-dicarboxylic acid $\left(\mathrm{H}_{2} \mathrm{~L}\right)$ are synthesized by the reaction between $\mathrm{Ni}$ acetate and $\mathrm{H}_{2} \mathrm{~L}$ in aqueous solution at 25 and $80{ }^{\circ} \mathrm{C}$, respectively. ${ }^{242}$ From the same procedure with polyvinylpyrrolidone (PVp) used as a surfactant, another respective micron size $\mathrm{Ni}(\mathrm{II}) \mathrm{CP}\left[\mathrm{NiL}\left(\mathrm{H}_{2} \mathrm{O}\right)_{2}\right]_{n}$ is obtained at 25 and at $80{ }^{\circ} \mathrm{C}$ (Fig. 22).

Very important is the fact that surfactants can be capping agents or inhibitors, thus providing steric stabilization, which leads to the formation of nanoparticles and has a different effect on various faces of a crystal causing growth anisotropy of MOF crystals. In this casem surfactants slow down crystal growth rate, providing spatial stabilization of MOF nanoparticles $^{223,224}$ and relative value of different faces of a crystal, which leads to anisotropic growth. ${ }^{52,56,243,244}$ Capping agents, such as trisodium citrate, are used for limitation of growth of nanoparticles and stabilization of their aggregation. Their effect is, as a rule, not long, though using trisodium citrate with Lee and Meisel method can bring to particles, which are stable for a month.

3.1.8. In situ spacer synthesis. The approach called 《in situ spacer synthesis $\rangle$, in which organic spacers are generated in reaction medium in situ from initial materials, attracts more interest in the recent years.

As an example, we shall consider solvothermal reaction of $\mathrm{Pb}$ acetate with flexible 1,3-bis(4-pyridyl-3-carboxyl)-propane $\left(\mathrm{H}_{2} \mathrm{~L}\right)$ under different conditions of synthesis through in situ ligand transformation reaction, which give three genuine coordination polymorphs $\left[\mathrm{PbL}^{2-}\right]_{n}, \quad\left[\mathrm{~Pb}_{3} \mathrm{~L}_{3}{ }^{2-}\right]_{n}$ and $\left[\left(\mathrm{Pb}_{2} \mathrm{~L}^{2-}\right) \cdot 2 \mathrm{H}_{2} \mathrm{O}\right]_{n}$ (Fig. 23). ${ }^{245}$ In these compounds, $\mathrm{L}^{2-}$ ligand shows different coordination conformations and regimes adjusted to different synthesis conditions, including temperature of reaction, cooling rate and dopants, and designs various architectures linking different building units. Two first polymorphs show 3D framework with $1 \mathrm{D}$ channels built from binuclear ring-like $\left[\mathrm{Pb}_{2}\left(\mathrm{~L}^{2-}\right)_{2}\right]$ and binuclear semiring-like $\left[\mathrm{Pb}_{2} \mathrm{~L}^{2-}\right]$ units, respectively. The last polymorph also has 3D architecture; however, it is built from binuclear ring-like $\left[\mathrm{Pb}_{2}\left(\mathrm{~L}^{2-}\right)_{2}\right]$ units interconnected with $\mathrm{L}^{2-}$ ligand.

We shall also notice CPs with $\left[\mathrm{Cu}(\mathrm{II})_{2}\left(\mu_{3}-\mathrm{L}\right)_{2}\left(\mu_{1}-\mathrm{Cl}\right)_{2}\right]_{n},[\mathrm{Cu}(\mathrm{I})$ $\left.\mathrm{Cu}(\mathrm{II})\left(\mu_{2}-\mathrm{L}\right)_{2}\left(\mu_{2}-\mathrm{Cl}\right)\left(\mathrm{H}_{2} \mathrm{O}\right)\right]_{n}$ and $\left[\mathrm{Cu}(\mathrm{I})_{2} \mathrm{Cu}(\mathrm{II})\left(\mu_{2}-\mathrm{L}\right)_{2}\left(\mu_{3}-\mathrm{I}\right)_{2}\left(\mathrm{H}_{2} \mathrm{O}\right)\right]_{n}$ units based on chelating ligand of 2-pyrazinecarboxylate (HL), which is formed in situ by hydrolysis of the initial $N, N^{\prime}-(5,7-$ dihydro-1,3,5,7-tetraoxobenzo[1,2-c:4,5- $\left.c^{\prime}\right]$ dipyrrole-2,6(1H,3H)diyl)bis-(9CI)-3-pyrazinecarboxamide upon its reaction with $\mathrm{Cu}(\mathrm{II})$ chloride, iodide, and perchlorate, respectively. ${ }^{246}$ The first CP has 2D double-layer structure, which can be considered as a binodal $(3,3)$-connected network, the second polymer is $(4,4)$ net, and the third CP has a 3D open framework built by linking 1D sawtooth chains of $\mathrm{Cu}(\mathrm{II})$ iodide clusters with $\left[\mathrm{Cu}(\mathrm{II})\left(\mu_{2}-\mathrm{L}\right)\right]$ units. It is interesting that all compounds cannot be synthesized directly using $\mathrm{HL}$ and the respective $\mathrm{Cu}$ ions as initial reagents under the same conditions of reaction. Therefore, the structural diversity of this system is adjusted in situ by hydrolysis reactions.

\subsection{Alternative synthesis routes}

In hydro(solvo)thermal processes, considerable volumes of a solvent are necessary to produce great quantities of CP for commercial using, which is environmentally unfavorable, and makes products costly. It is also necessary to accelerate crystallization process and generate uniform crystals with diminished size; therefore it is important to develop easily available, non-expensive, fast, and commercially viable synthetic routes. Many efforts were taken for development of alternative methods of CP synthesis, such as electrochemical, sonochemical, microwave, and mechanochemical methods. These methods provide possibilities for MOF synthesis in reduced time and with high quality, which is favorable for commercial application of MOFs. In this view, detailed studies were performed for optimization of MOF synthesis conditions to obtain high yields of solid products for industrial applications. ${ }^{243,244}$

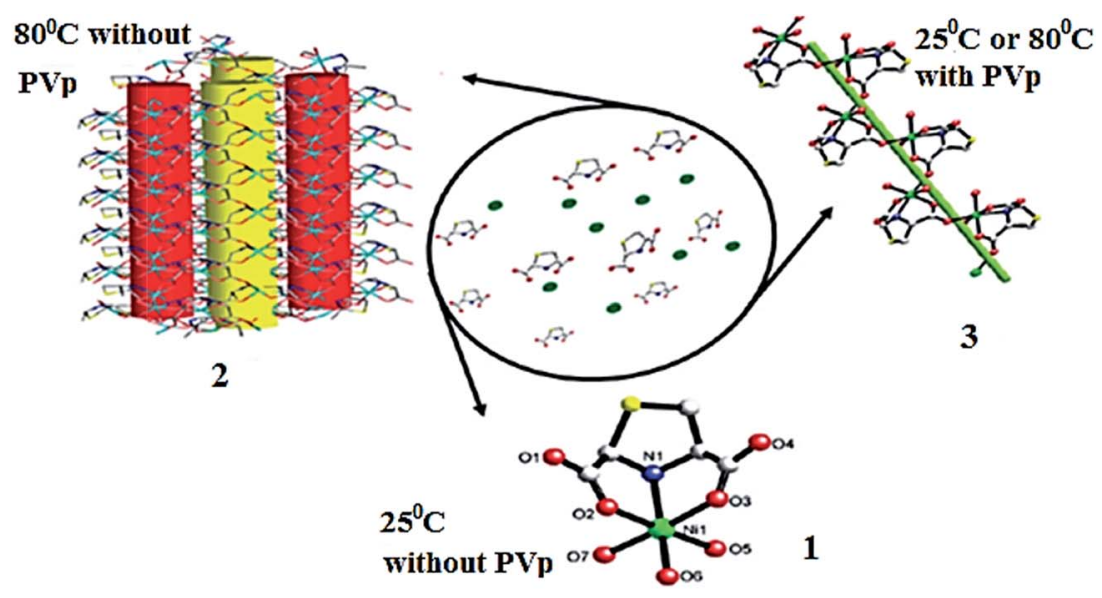

Fig. 22 Schematic representation of the generation of discrete 1, 3D framework 2, and 1D left helical chain 3 triggered by temperature or polyvinylpyrrolidone. [Reprinted with permission from ref. 242. Copyright 2012, American Chemical Society.] 
(a)
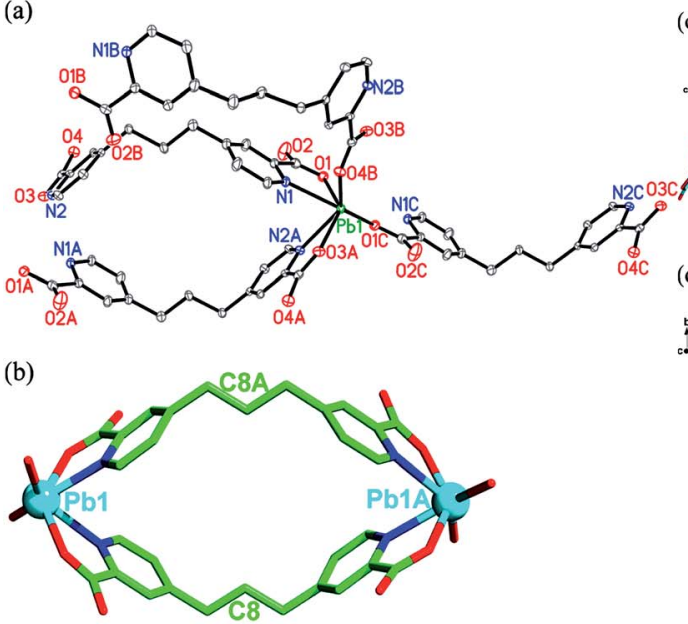

(c)

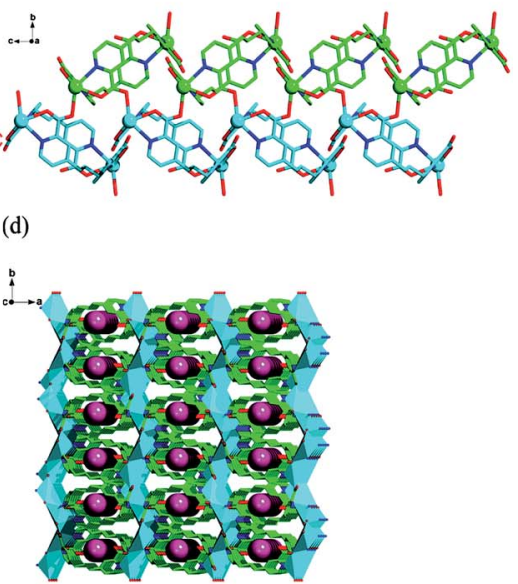

(e)

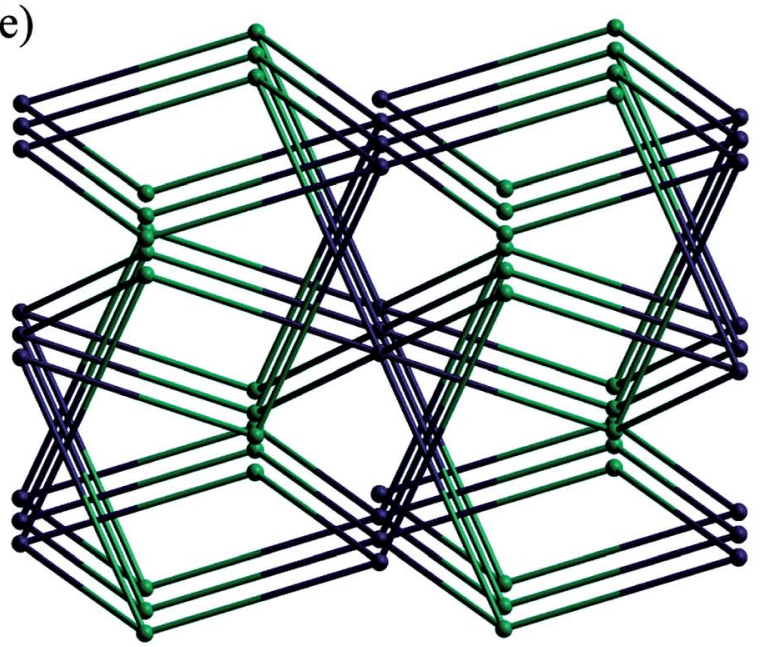

Fig. 23 (a) View of the coordination environments of the $\mathrm{Pb}_{1}$ center in CP. (b) View of one dinuclear [Pb $\mathrm{PL}_{2}{ }^{-2}$ ] ring-like unit of $\mathrm{CP}$. (c) View of a section of the 1D double chain extending along the $c$ axis. (d) View of a 3D porous structure in CP along the $c$ axis. (e) Topological view showing the equivalent 3D framework of CP. The purple pillars represent the $1 \mathrm{D}$ channel in $\mathrm{CP}$. Atom color codes: $\mathrm{Pb}, \mathrm{Cyan} ; \mathrm{O}, \mathrm{red} ; \mathrm{N}$, blue; and $\mathrm{C}$, green. [Reprinted with permission from ref. 245. Copyright 2012, American Chemical Society.]

3.2.1. Microwave-assisted synthesis. It is important to emphasize that microwave-assisted MOF syntheses are mainly focused on the following moments: acceleration of crystallization, formation of nanometer materials, improvement of purity of a final product, and selective synthesis of polymorphs. This is reached by direct heating solvents and increase in crystal nucleation rate. To optimize the reaction conditions, systematic studies of changes in the composition and process parameters (solvent, irradiation time, temperature of reaction, power level, the molar ratio of reagents, reagent concentration, etc.) were carried out. ${ }^{221}$ It should be noted that microwave irradiation makes it possible to accelerate synthesis of fine crystals as compared with conventional heating. On the whole, advantages of this method are high efficiency, phase selectivity, particle size diminishing, and morphology control.

As a typical example, we consider microwave-assisted synthesis of Co-MOF-74 under different conditions, in particular, temperature of synthesis, power level of microwave irradiation, and time of synthesis. ${ }^{221}$ Optimized Co-MOF-74 was synthesized at $180^{\circ} \mathrm{C}$ at $180 \mathrm{~W}$ power in a microwave oven during $1 \mathrm{~h}$, which resulted in the highest textural properties $\left(1314 \mathrm{~m}^{2} \mathrm{~g}^{-1}\right.$ of BET surface area) with high product yield (about $76 \%$ per ligand). Co-MOF-74 ( $c a .50 \mu \mathrm{m}$ long and $8 \mu \mathrm{m}$ wide) obtained by microwave heating was smaller in size than the solvothermally prepared Co-MOF-74 (ca. $300 \mu \mathrm{m}$ long and $70 \mu \mathrm{m}$ wide).

Another interesting example is production of rare earth based CP submicrospheres from pyridine-2,5-dicarboxylic acid and $\mathrm{M}\left(\mathrm{NO}_{3}\right)_{3}$ using the method of light microwave heating during 5 min with DMF as a solvent. ${ }^{247}$ Submicrospheres have diameters 100-400 nm, and the microspheres' surface is smooth. Strawsheaf-like Tb-based CP architectures are successfully synthesized using a simple and ecological microwave heating in great scales during 15 min without any template or surfactant using 1,2,4,5benzenetetracarboxylic acid as organic building unit. ${ }^{248}$ The measured specific surface area of Tb-based CP was $152.51 \mathrm{~m}^{2} \mathrm{~g}^{-1}$. 
Individual straw-sheaf has length in the range 70-90 $\mu \mathrm{m}$, and average diameter in the range 5-8 $\mu \mathrm{m}$.

3.2.2. Electrochemical synthesis. In order to exclude metal salts usage, metal ions are continuously integrated via anode dissolution into the reaction mixture, which contains dissolved spacer molecules and conducting salt. ${ }^{249}$ Other advantage of electrochemical route is a possibility to launch a continuous process and a possibility to obtain higher content of solids as compared with batch reactions. Undoubtedly, electrochemical MOF synthesis provides alternative method of synthesis, which does not require additional external source of heat, however, it is too expensive to be used at industrial scale. It is important that this approach works through formation of the reagent ions in a solution, thus it is unnecessary to use bases. It is worth noticing that electrochemical methods suits well for formation of MOF membranes due to intrinsic coating reached on scaffolds due to a MOF product deposition.

3.2.3. Mechanochemical synthesis. In mechanochemical synthesis, the mechanical break of intramolecular bonds followed by chemical transformation occurs. ${ }^{\mathbf{1 2 0}}$ Mechanochemical synthesis is a promising alternative method for systematic and large scaled CP production. Its advantages include the following: lowering of contamination, low cost, simplicity of the process, easiness of processing, efficient reaction rate, selectivity, and solution of a problem of low solubility of reagents. Mechanosynthesis or milling is currently used as fast, scaled, and potentially non-toxic way of MOF synthesis. MOFs can also be obtained using mechanochemistry, i.e. grinding of two or more solids using a mechanical ball mill and thus avoiding use of solvents. ${ }^{250}$ This is very aggressive technique, which makes it possible to obtain products from micro- to nanocrystals at room temperature in a shorter time.

Using twin screw and single screw extruders has been shown for continuous synthesis of different CPs, including Ni(salen)based. ${ }^{251}$ Quantitative conversions are reached with the formation of products at the $\mathrm{kg} \mathrm{h}^{-1}$ rates, which after activation show surface areas and volume of the pores equivalent to materials obtained by conventional solvent-based method.

High proton conductivity material has been obtained using economical and ecologically pure mechanochemistry. ${ }^{252}$ The reaction is a new synthetic strategy with respect to materials related to technology of fuel cells.

3.2.4. Sonochemical synthesis. It is important that using ultrasound of high intensity provides easy, ecological, and universal synthetic tool for production of nanostructured materials, which are often unavailable for conventional methods. ${ }^{253}$

Sonochemical methods through uniform and accelerated nucleation also provide decrease in crystallization time and far less sizes of particles, than those provided by conventional solvothermal synthesis. For this purpose, a solution of mixture of substrates for this MOF structure is input in a horn-type Pyrex reactor equipped with an ultrasonic bar with regulated output power without external cooling. As an example, we shall consider sonochemical synthesis of nanometer MOF with $\left[\mathrm{Pb}_{2}\left(\mathrm{~N}_{3}\right)\left(\mathrm{NO}_{3}\right) \mathrm{Q}_{2}\right]$ unit, where HQ is 8-hydroxyquinoline. ${ }^{254}$
Sonochemically are also obtained nanostructures of CPs with $\left[\mathrm{Pb}_{2} \mathrm{~L}_{4}\right]_{n}\left(\mathrm{HL}=2\right.$-pyrazinecarboxylic acid) and $\left[\mathrm{Pb}\left(\mathrm{L}^{\prime}\right)_{2}\right]_{n}$ $\left(\mathrm{HL}^{\prime}=2\right.$-quinolinecarboxylic acid) units. $^{255}$

There is an interest in sonochemical synthesis of nano-sized $\mathrm{Pb}$ (II) CP with $1 H$-1,2,4-triazole-3-carboxylate ligand. ${ }^{256}$

High quality Mg-MOF-74 crystals $\left(1.640 \mathrm{~m}^{2} \mathrm{~g}^{-1}\right.$ BET surface area) with particle size about $0.6 \mu \mathrm{m}$ are successfully synthesized during $1 \mathrm{~h}$ by sonochemical method after addition of triethylamine as a deprotonating agent. It is interesting that mesopores are formed, probably, due to competitive linking triethylamine to $\mathrm{Mg}$ (II) ions. ${ }^{220}$

\section{Post-synthetic transformations}

In the recent years, post-synthetic transformations have become powerful tools for synthesis of the CPs with chelated units, which cannot be reached with de novo synthesis due to limited linker solubility, thermal stability, chemical stability, functional group compatibility, and undesired intervention between metal ions and linker of functional fragments during MOF assembling. Removal or substitution of solvents and guest molecules, action of chemically active vapors and external stimuli, such as heat, light or mechanochemical force, induce such structural transformations, and they often manifest themselves in different physical properties, such as color, magnetism, luminescence, chirality, porosity, etc. because of a change in coordination number and geometry, dimensionality, interpenetration, etc. More radical transformations associated with exchange of metal ions, pillar ligands, and insertions of additional ligands between layers were also represented.

Post-synthetic transformations include post-synthetic modification (PSM), post-synthetic deprotection (PSD) and post-synthetic exchange (PSE).

\subsection{Post-synthetic modification}

Post-synthetic modification (PSM) is a single-crystal to singlecrystal (SC-SC) transformation and is the process including transformation of side groups on surface or in pores of MOFs in order to change physical or chemical properties. This can be reached with chemical reaction, adsorption, ligand or metal exchange, light, and pressure stimuli. Post-synthetic MOF modification opens new dimension of structural transformations, which cannot be reached by conventional synthesis.

There are metal centers and initial ligands with functional groups, which can take part in a reaction under normal conditions of MOF synthesis for the preparation of MOFs with specified crystal structures and pore sizes. Then the functional groups on ligands can react with post-synthetic reagents to obtain final desired functionalities. ${ }^{257}$ PSM MOFs can be realized using multiple interactions, among which covalent and coordination bonds are the most important. Post-synthetic methods, which modify MOFs with chemical reagents with the lattice structure unchanged, make it possible to integrate functional groups in MOFs. ${ }^{258}$ 

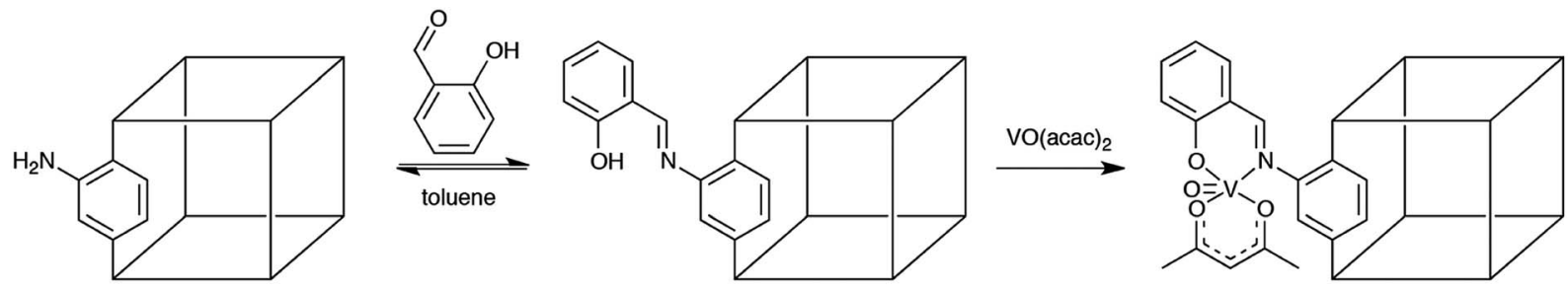

Scheme 18 Scheme illustrating for the functionalization of IRMOF-3 with salicylaldehyde and subsequent binding a vanadyl complex. [Reproduced from ref. 258 with permission from The Royal Society of Chemistry.]

Chelating groups can be introduced more easily by post-than by presynthetic functionalization in MOFs since they may compete in the coordination to inorganic nodes. There are various examples in the literature that show how to produce chelating groups by PSM. It is important that potential chelating fragments can be introduced in MOFs by covalent post-synthetic modification, so that they do not cause interference to the synthesis, but are still available for further coordination modification.

In particular, universality of 2-amino-1,4-benzenedicarboxylate as a building block has been showed: it can be smoothly subjected to PSM in three different prototypic MOF topologies: IRMOF-1, DMOF-1 ( $\mathrm{D}=$ dabco), and UMCM-1. The important features of PSM on IRMOF-3 include (1) facile introduction of a wide range of functional groups using simple reagents (e.g., anhydrides and isocyanates), (2) the introduction of multiple (as many as four different) substituents into the MOF lattice, and (3) control over reaction conditions to preserve the crystallinity and microporosity of the resultant MOFs.

As a typical example, we note imine condensation of IRMOF3 wetted in toluene solution and excess of salicylaldehyde during 7 days, which has given a PSM-product with $\sim 13 \%$ conversion of amino groups. ${ }^{259}$ Then $\mathrm{V}(\mathrm{O}) \mathrm{acac}_{2}$ was loaded in the framework for coordination with the salicylidene groups, and the second step was almost quantitative (Scheme 18). In this case, post-synthetic derivatization of a porous material gives functionalized material, which links a metal complex $\mathrm{VO}(\mathrm{acac})_{2}$ in contrast to non-functionalized precursor, which is inactive for complex linking.

In conversion of roles, aldehyde can be a part of MOF, as in the case of ZIF-90 (isostructural to ZIF-8, but with a tag aldehyde group). ${ }^{260}$ Specifically, reduction of aldehyde to alcohol functionality was successfully achieved by reacting ZIF-90 with $\mathrm{NaBH}_{4}$ in methanol at $60{ }^{\circ} \mathrm{C}$ for $24 \mathrm{~h}$ to give ZIF-91 with about $80 \%$ conversion (Fig. 24A). The chemical versatility of the aldehyde group was highlighted by performing another organic transformation on ZIF-90. Reaction of ZIF-90 with ethanolamine in methanol at $60^{\circ} \mathrm{C}$ gave ZIF-92 (Fig. 24B). Quantitative conversion to the imine was completed within three hours.

Tandem PSM is useful, since it can be realized in one-pot synthesis. PSM MIL-101(Fe)- $\mathrm{NH}_{2}$ with Py-2-carboxaldehyde and $\mathrm{NiCl}_{2}$ in the one-pot reaction has given $\sim 30 \%$ conversion of amino groups into pyridine imino groups (Scheme 19). ${ }^{35}$ Nickel centers are integrated as catalytic sites into pores by coordination with a nitrogen atom of imino group and a pyridine nitrogen atom. Ni complex is formed, first of all, to stop any competition with aldehyde reagent for amino groups.

Additional set of examples is observation that traditional batch hydro(solvo)thermal methods of synthesis, as a rule, exclude direct incorporation of free-base Pp as a spacer in MOF, since spacers spontaneously link metal ions present as building blocks and nodes. ${ }^{63}$ A promising particular solution of the abovementioned problem is post-synthetic treatment afterwards renamed and generalized in PSM, i.e. chemical substitution (atom, ion or functional group, addition, substitution or transformation) of a framework without a change in parent topology. Really, a task with participation of metal-free Pp or salen-based spacers was first met by assembling metallized spacer in variants of compounds, and then by substitution of non-structural (i.e. spacer-localized) metal ions with a pair of protons. The second stage of PSM accepts new metal ions.
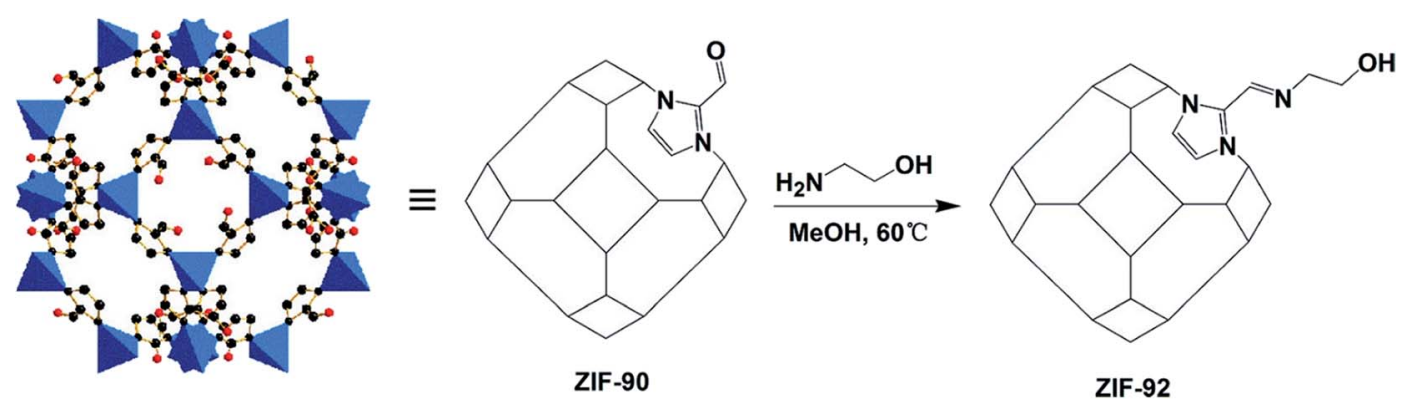

Fig. 24 Crystal structure of ZIF-90 and the covalent postsynthetic functionalization of aldehyde groups with ethanolamine to give ZIF-92. Color scheme: for $\mathrm{C}$ atoms, black; for $\mathrm{N}$ atoms, green; for O atoms, red. [Reprinted with permission from ref. 260. Copyright 2017, American Chemical Society.] 

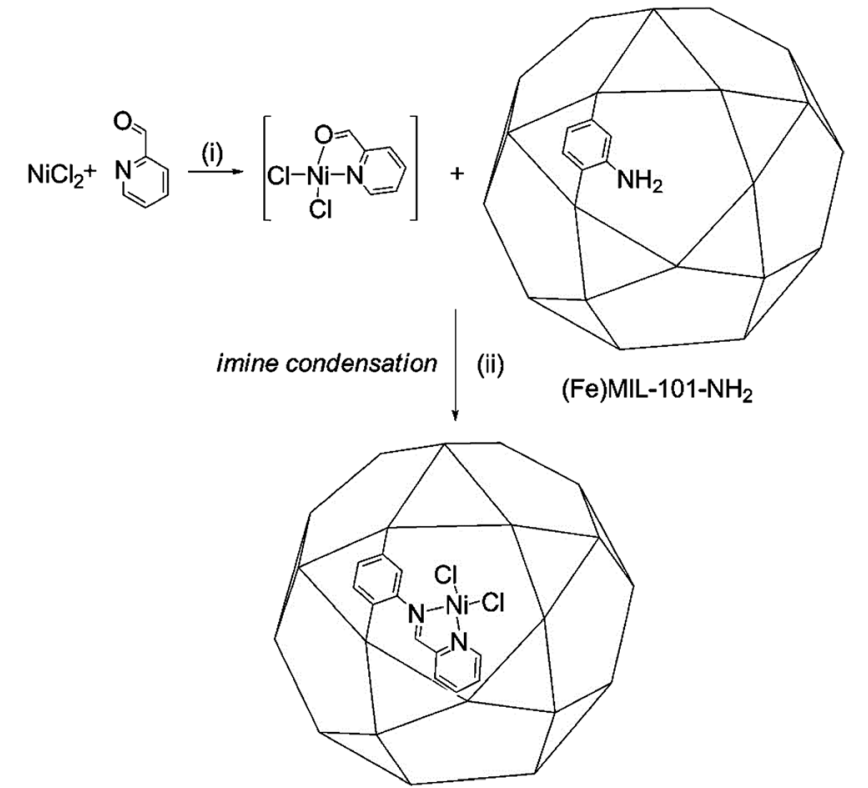

10\% functionalization: 10Ni@(Fe)MIL-101 30\% functionalization: 30Ni@(Fe)MIL-101

Scheme 19 One-pot synthesis of the MOF-anchored nickel complex $\mathrm{Nia}(\mathrm{Fe}) \mathrm{MIL}-101$. [Reprinted with permission from ref. 35. Copyright 2013, American Chemical Society.]

It should be noted that covalent modification of bridging ligands is studied in detail. ${ }^{49,261}$ One of the most widely applied PSM approaches is modification of organic spacers using chemical reactions with preservation of a lattice structure. In particular, azide-functionalized MOFs are used for click reactions. ${ }^{262} \mathrm{~A}$ special interest is MOF, where metal centers are subjected to modification. Also a possibility is demonstrated of PSM via coordination of additional metal ions with centers in bridging ligands ${ }^{263}$ and addition or removal of metal ions in metal centers. ${ }^{264}$

Of particular note is the linker installation method, in which a stable Zr-MOF with inherent missing linker sites, namely, PCN-700, with coordinatively unsaturated $\mathrm{Zr}_{6}$ clusters was employed and linkers bearing different functional groups were post-synthetically installed (Fig. 25). ${ }^{265}$ At first, the bpy-5, $5^{\prime}$ dicarboxylate and terphenyl-4, $4^{\prime}$-dicarboxylate were sequentially installed by treating PCN-700 crystals with solutions of bpy-5, $5^{\prime}$ dicarboxylate and terphenyl-4,4'-dicarboxylate in DMF at $75^{\circ} \mathrm{C}$. The modified MOF bears open bpy sites that readily react with $\mathrm{CuI}$ in acetonitrile to form the metalated framework. According to the crystal structure, the $\mathrm{Cu}^{+}$center is chelated by a bpy group and further coordinated with an $\mathrm{N}$ from acetonitrile and an $\mathrm{I}^{-}$ ion as a counter-ion. The $\mathrm{Cu}^{+}$center is arranged around the 1D channel along the $c$-axis, whereas the terphenyl-4, $4^{\prime}$-dicarboxylate controls the accessibility of the $\mathrm{Cu}^{+}$center by partially blocking the channels. Therefore, the size selectivity of the whole material can be tuned by changing the size of substituents on the terphenyl-4,4'-dicarboxylate linker. In particular, the terphenyl-4,4'-dicarboxylate linker was functionalized by methyl groups, phenyl groups, and hexyl groups, which is expected to result in different selectivity toward the substrates.

Unsaturated metal sites were used in an alternative method, in which MOF with $\left[\mathrm{Cu}_{3} \mathrm{~L}_{2}\right]$ unit $(\mathrm{L}=1,3,5$-benzenetricarboxylate) was modified with en followed desolvation under vacuum to prepare free coordinating fragments. ${ }^{266}$

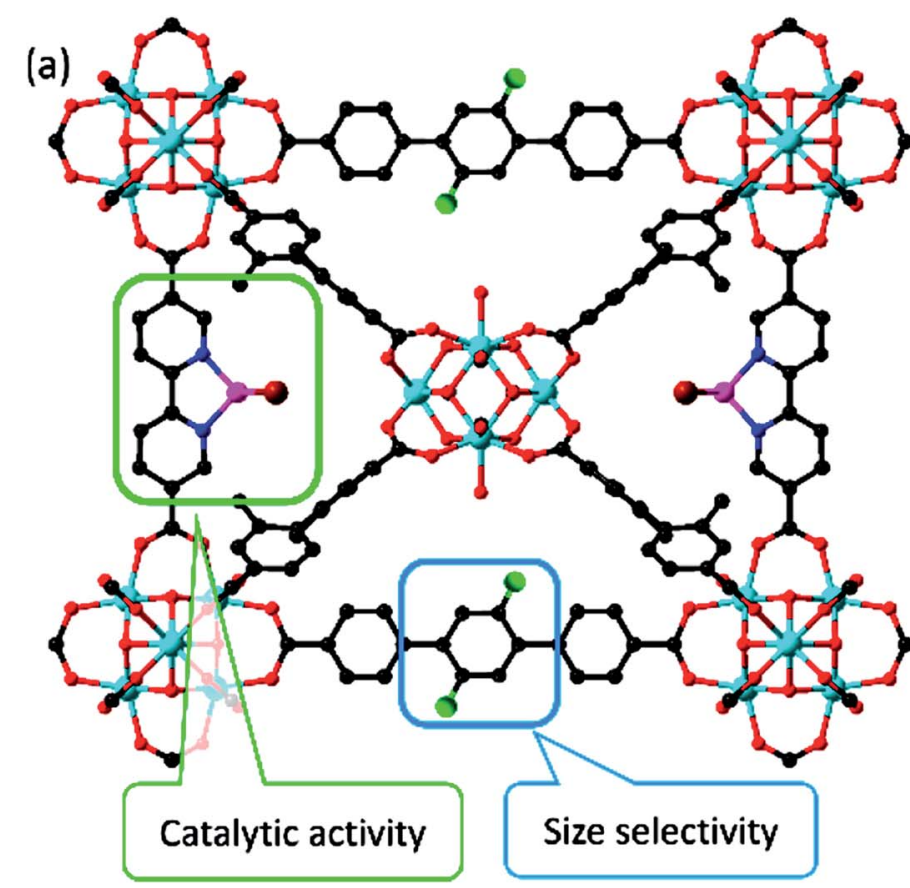

(b)

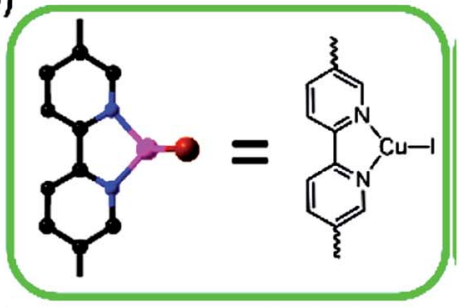

(c)

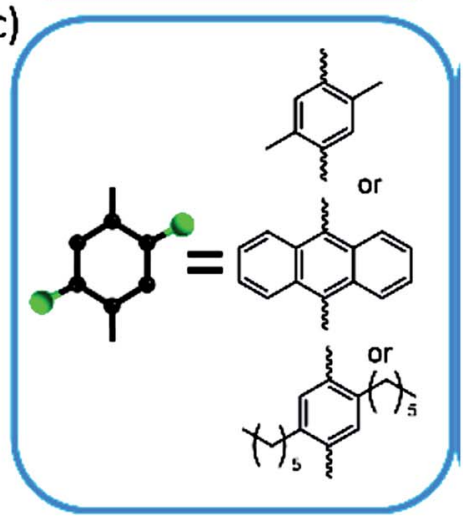

Fig. 25 (a) Size-selective catalytic system for aerobic alcohol oxidation reaction built in PCN-700 through linker installation. (b, c) Structure of catalytic center and size-selective moiety. Coordinated $\mathrm{CH}_{3} \mathrm{CN}$ on the $\mathrm{Cu}$ is removed for clarity. [Reprinted with permission from ref. 265. Copyright 2016, American Chemical Society.] 


\subsection{Post-synthetic deprotection}

PSD is useful for synthesis of functional MOFs, such as those which have groups with a trend to metal complexes formation in direct synthesis. The process includes elimination of complexity in MOF and in production of big cavities. PSD is sometimes also called protection, complexation, and deprotection. ${ }^{267}$

By now, PSD just begins to draw attention as a post-synthetic method of functionalization. The concept of this method is that a protective functional group is incorporated as an organic spacer, and then the protective group is removed by the postsynthetic method. It should be noted that post-synthetic MOF modification is realized for the preparation of single-site catalysts. $^{268}$

Protection-deprotection methods open new possibilities for 〈unlock $\rangle$ of protected functionalities for production of highly active and/or coordinating fragments on MOFs. This can facilitate immobilizing metal-organic catalysts in MOF cavities. ${ }^{269}$

\subsection{Post-synthetic exchange}

Post-synthetic exchange (PSE) also known as a building block replacement $^{263}$ includes replacement of basic structural components of preliminary assembled MOFs. PSE methods include (I) solvent-assisted linker exchange (SALE), (II) nonbridge ligand replacement, and (III) transmetalation. These one-stage or tandem PSE processes suppose exchange of key structural MOF components, which, in turn, should provide evolution of initial MOF structures for MOFs design consisting of absolutely new components, supposedly, using SC-SCtransformations.

SALE is one of most widely used PSE approaches for adjustment of MOF functionality. SALE realization, as a rule, involves holding MOFs crystals in a solution of a spacer substitution candidate in a thorough chosen solvent. ${ }^{270}$ It is worth noticing that SALE proceeds through exchange of ligands in undamaged MOFs, but not by solution and re-crystallization. Therefore, topology of a parent MOF is reproduced in a daughter structure. SALE was demonstrated for a range of MOFs, ${ }^{270-273}$ including such, seemingly, inert compounds as ZIFs and, which is more surprising, UiO- $66,{ }^{274,275}$ the material, which is characterized by unusually strong $\mathrm{M}-\mathrm{L}$ bonds.

Thus, modification of UiO-66 is executed with catechine using SALE in DMF/ $\mathrm{H}_{2} \mathrm{O}$ solution during 2 days at $85{ }^{\circ} \mathrm{C}$. After

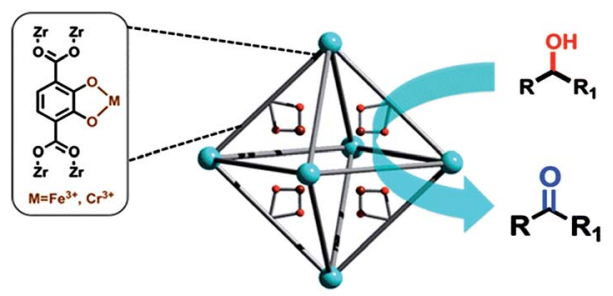

Fig. 26 Schematic view of catechol-functionalized UiO-66 after metalation for catalytic oxidation of alcohols to ketones. [Reprinted with permission from ref. 276. Copyright 2014, American Chemical Society.] metalation, Cr-metallized MOFs were synthesized (Fig. 26) ${ }^{276}$ Importantly, insulated metal-monocatecholato fragment is achieved in very strong MOF by two principally different strategies: PSD and PSE. As compared with PSD, PSE is easier and more efficient approach to functioning for MOFs access, which cannot be synthesized directly under solvothermal conditions. Metalation of catechine functionality remained in MOFs led to unprecedented Fe-monocatechelato and Cr-monocatechelato types.

PSE was also employed as a strategy to introduce dimercapto functionality into UiO-66. ${ }^{277}$ In particular, PSE was performed by incubating solid UiO-66 in an aqueous solution of 2,3dithiocatechine-1,4-benzenedicarboxylic acid (tcat- $\mathrm{H}_{2}$ bdc) for $24 \mathrm{~h}$ at $85^{\circ} \mathrm{C}$. The linker-exchanged thiocatechol material, UiO66-TCAT, was isolated as a yellow microcrystalline powder using centrifugation. Immobilized thiocatechol ligands on the UiO-66-TCAT provide an excellent platform to achieve accessible and unsaturated mono(thiocatecholato) metal centers (Scheme 20). The metalation of $40 \%$ thiocatecholfunctionalized UiO-66-TCAT using $\mathrm{Pd}(\mathrm{OAc})_{2}$ in $\mathrm{CH}_{2} \mathrm{Cl}_{2}$ afforded dark-brown solids. The crystallinity of UiO-66-PdTCAT was maintained upon metalation. It is important to notice that Pdmetalated MOFs are efficient, heterogeneous, and recycled catalysts for regioselective functionalization of $\mathrm{sp}^{2} \mathrm{C}-\mathrm{H}$ bond. This material is a rare example of chelating-assisted $\mathrm{C}-\mathrm{H}$ functionalization performed by a MOF catalyst.

Consecutive process of post-synthetic exchange of ligands was used for production of a range of mono-, di-, and trifunctionalized mesoporous MOFs. ${ }^{278}$ Using this process, orthogonal functional groups are established, and after that post-synthetically modified with a dye and quencher molecules, illustrating the level of structural and functional complexity that can be achieved within this system. It is important that the degree of completion and the relative ease of ligand exchange can depend on the lability of the M-L bond within the MOF.

An extended Pp MOFs family is developed, which includes directly variety of $\mathrm{M}-\mathrm{Pp}$ through $\mathrm{M}-\mathrm{Pp}$ and M-bipyridyl Pp as building units, which have large available channels and active metal sites (Scheme 21). ${ }^{\mathbf{1 1 0}}$

A simple substitution of a solvent is basic for PSM chemistry. As a typical example, we note a solvent replacement in MOF with $\left[\mathrm{CdL}_{2}\left(\mathrm{ClO}_{4}\right)_{2}\right]_{n}$ unit, where $\mathrm{L}=(S)$-2,2'-diethoxy-1,1'-binaphthyl6,6'-bis(4-vinylpyridine), in which ethanol was reversibly exchanged with benzene in chiral 1D hexagonal channel. It is assumed that this MOF has a good potential for heterogeneous asymmetric catalysis, since the structure can be supported after replacement of a solution. ${ }^{279}$

Post-synthetic transmetalation has become a prominent way of synthesis of new crystalline materials based CPs and is especially powerful for orientation of metastable phases, which are unavailable for conventional high temperature synthetic methods. ${ }^{258,264,280-282}$ Through integration of cations in certain coordination, post-synthetic transmetalation provides rational design of physical properties and chemical reactivity, which otherwise can be physically and chemically inactive. Clear structure-function understanding is required for design of these materials, though their high structural complication undermines 


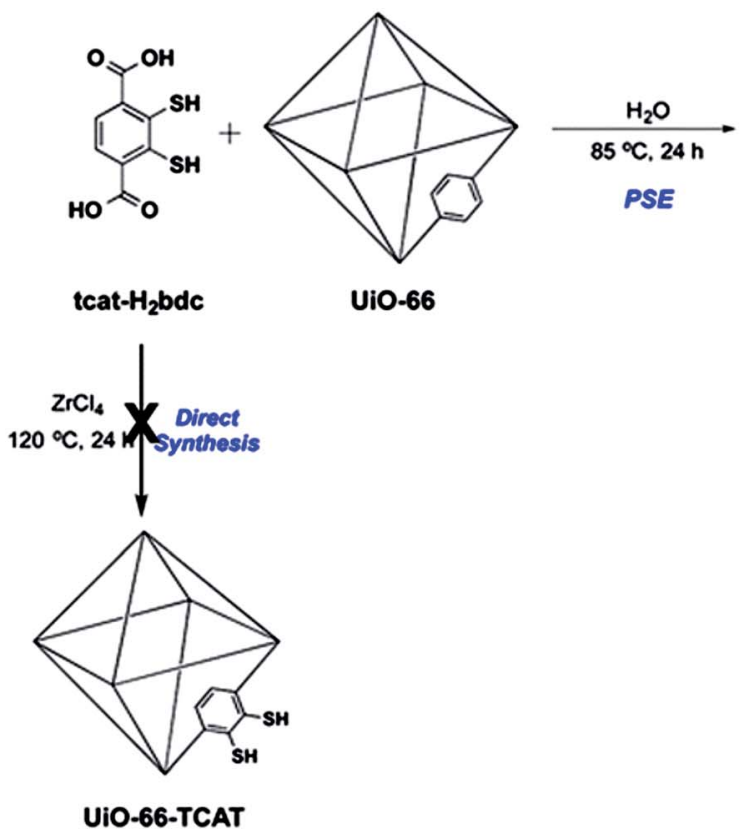

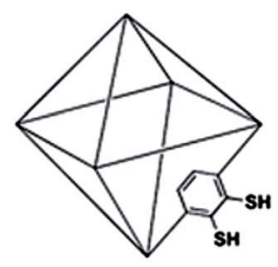

UIO-66-TCAT

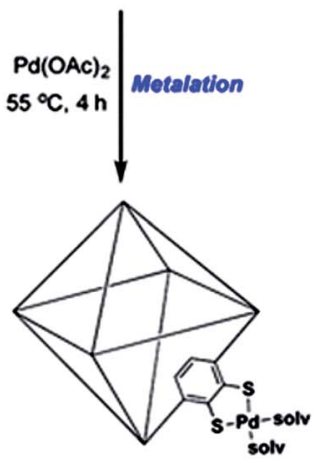

UIO-66-PdTCAT Catalytically Active

Scheme 20 Schematic view of the synthesis of UiO-66-TCAT and UiO-66-PdTCAT. [Reprinted with permission from ref. 277. Copyright 2015, American Chemical Society.]

at the atomic level structural characterization. Thus, relative occupation of cation on two crystallographically different metal sites in $\mathrm{Fe}(\mathrm{II})-\mathrm{Cu}(\mathrm{II})$-, and $\mathrm{Zn}(\mathrm{II})$-exchanged versions of microporous MnMnL MOF ( $\mathrm{L}=1,3,5$-benzenetristetrazolate), was established. It is important that using dispersion differences between $\mathrm{Mn}, \mathrm{Fe}, \mathrm{Cu}$, and $\mathrm{Zn}$, degree and localization of cation exchange are determined. In addition, the replacement degree for $\mathrm{Mn}$ (II) depends on the identity of the substituting metal. ${ }^{283}$

Different amounts of Co and Ni were substituted in Mg-MOF74 using one-pot solvothermal reaction. ${ }^{284}$ Based on elemental analysis, $\mathrm{Co}$ and $\mathrm{Ni}$ were more efficiently incorporated into MOF-74 framework from solution than $\mathrm{Mg}$ was. In addition, temperature of the reaction has stronger effect on the final composition of metal in these mixed metallic MOF-74 structures than a solvent composition of the reaction.

Fast room-temperature cation exchange is also observed during soaking of $\mathrm{CP}$ with $\left[\mathrm{Pb}_{2} \mathrm{Cl}_{2}(\mathrm{HL})_{2}\left(\mathrm{H}_{2} \mathrm{O}\right)_{2}\right]_{n}$ unit based on pyrazine-2,3-dicarboxylic acid $\left(\mathrm{H}_{2} \mathrm{~L}\right)$ in $\mathrm{Cu}(\mathrm{II})$ aqueous solutions (Scheme 22). ${ }^{127}$

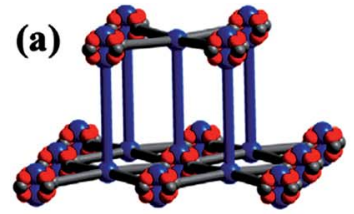

AB

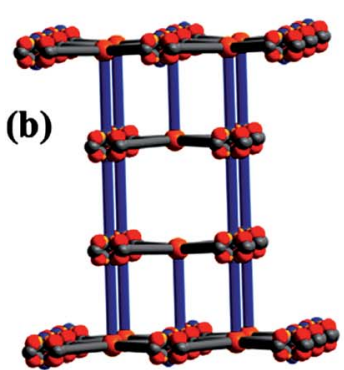

ABBA

PPF-20
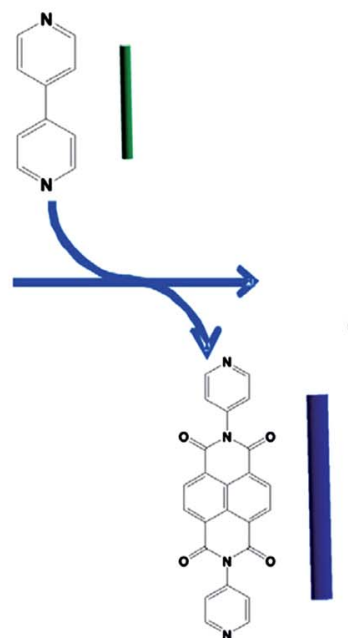

(c)

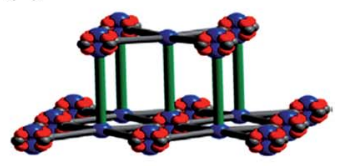

AB

PPF-27

(d)

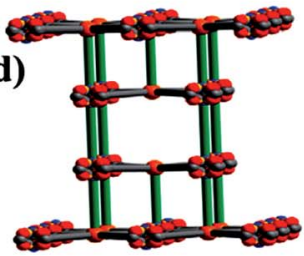

ABBA

\section{PPF-4}

Scheme 21 Schematic illustration of the introduction of 4,4'-bipy to the crystals of PPF-18 (a) and PPF-20 (b) for the transformation of PPF-27 (c) and PPF-4 (d), respectively. [Reproduced from ref. 110 with permission from The Royal Society of Chemistry.] 


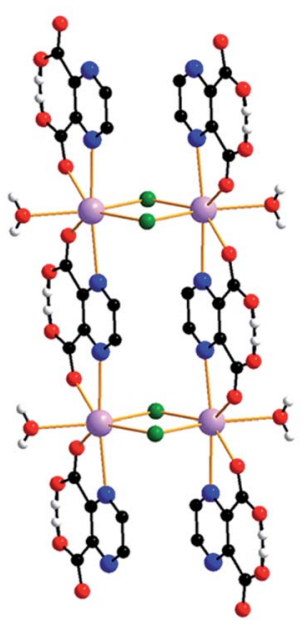

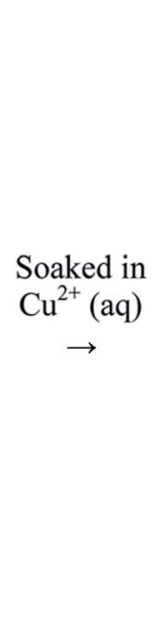

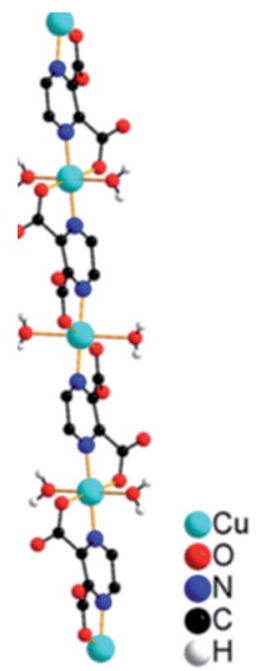

Scheme 22 Cation exchange by simple soaking of $\mathrm{Pb}($ (I) MOF into an aqueous solution of $\mathrm{Cu}(\mathrm{II})$ cation at room temperature. [Reprinted with permission from ref. 127. Copyright 2015, American Chemical Society.]

Series of metallosalen-based MOFs is prepared using postsynthetic modification of $\mathrm{Mn}^{\mathrm{III}} \mathrm{SO}-\mathrm{MOF}, \mathrm{Mn}^{\mathrm{III}}$ (salen)-based MOF. ${ }^{285}$ Treatment of $\mathrm{Mn}^{\mathrm{III}} \mathrm{SO}-\mathrm{MOF}$ with $\mathrm{H}_{2} \mathrm{O}_{2}$ leads to removal of $\mathrm{Mn}^{3+}$ ions from salen pillar, which then can be remetalated with different metal precursors with formation of isostructural MSO-MOF materials (Scheme 23). Presence of new metallosalen pillars in MSO-MOF is entirely confirmed. It is important that remetalated $\mathrm{Mn}{ }^{\mathrm{II}} \mathrm{SO}-\mathrm{MOF}$ material reflects similar catalytic activity and porosity to initial MOF.

Exchange of metal cations within a MOF has also been demonstrated for M-Pp MOF. Thus, easy route to M-Pp-based nanoreactor with post-synthetic metal-ion exchange with active metal cations and catalytically inactive MMPF, which consists of nanoscopic multifaced cages is developed. ${ }^{60}$ Local coordination of the active metal center in Pp macrocycle after the metal-ion exchange was crystallographically identified.

It should be noted cationic PCN-223(Fe) formed by synthetic treatment of PCN-223, Pp Zr-MOF with shp-a network, which is excellent recycled heterogeneous catalyst for hetero-Diels-Alder reaction..$^{286}$

Two isostructural Zr-Pp and Hf-Pp MOF (FJI-H6 and FJI-H7) are rationally synthesized and built from $2.5 \mathrm{~nm}$ cubic cages. ${ }^{287}$

It is worth noticing that in FJI-H6 and FJI-H7 two nitrogen atoms of the Pp ring are not deprotonated, therefore metal ion of another type is integrated in the framework. Submergence of FJI-H6 or FJI-H7 single crystals in $0.5 \mathrm{M}$ solution of $\mathrm{Cu}\left(\mathrm{NO}_{3}\right)_{2}$ in DMF at the temperature $85{ }^{\circ} \mathrm{C}$ during $72 \mathrm{~h}$ leads to metalated FJI-H6(Cu) or FJI-H7(Cu). As was expected, both in FJI-H6 and FJI-H7 $\mathrm{Cu}(\mathrm{II})$ ions were inbuilt in Pp rings, and $\mathrm{Cu}(\mathrm{II})$ ions in plane-square $\mathrm{N}_{4}$ coordination with two axial sites were exposed, which is typical of bivalent metal ions in Pp MOFs.

Of interest are isostructural Pp MOFs, in particular, UNLPF$10 \mathrm{~b},-11$, and -12 , which consist of $\mathrm{In}^{\mathrm{III}}-\mathrm{Sn}^{\mathrm{IV}} \mathrm{Cl}_{2^{-}}$, and $\mathrm{Sn}^{\mathrm{IV}}-\mathrm{Pp}$ building units, respectively. ${ }^{288}$ Metalation with high valence metal cations ( $\mathrm{In}^{\mathrm{III}}$ and $\left.\mathrm{Sn}^{\mathrm{IV}}\right)$ considerably changes electronic structure of $\mathrm{Pp}$ macrocycle and provides high oxidization photoexcited state, which can be subjected to efficient reduction extinguishing processes to facilitate organic reactions.

It should be noted Pp-based MOF MMPF-5, in which each small cubicoctahedral cavity consists of faces of six $\mathrm{Cd}(\mathrm{II})$ metalated tetrakis(3,5-dicarboxyphenyl)porphine units interlinked by eight triangular $\mathrm{Cd}\left(\mathrm{CO}_{2}\right)_{3}$ fragments (Fig. 27a). ${ }^{60} \mathrm{In}$ MMPF- 5 crystal structure, a Cd(II) cation within Pp ligand ring is far from Pp plane (Fig. 27b), which points to weak linking Cd(II) cations in Pp macrocycle and on a possibility to replace big $\mathrm{Cd}(\mathrm{II})$ by a smaller cation of a bivalent metal. Submerging the MMPF-5 crystals into DMSO solution of $\mathrm{Co}\left(\mathrm{NO}_{3}\right)_{2}$ at $85{ }^{\circ} \mathrm{C}$ for two days gives $\mathrm{Co}(\mathrm{II})$-exchanged MMPF-5(Co). Complete substitution of $\mathrm{Cd}(\mathrm{II})$ by $\mathrm{Co}(\mathrm{II})$ is confirmed exclusively within $\mathrm{Pp}$ macrocycles, however, $\mathrm{Cd}(\mathrm{II})$ cations in the framework have remained untouched, possibly, due to their strong chelating with six carboxylic oxygen atoms. Therefore, small cubicoctahedral cavity in MMPF-5(Co) is distinguished by faces of six $\mathrm{Co}(\mathrm{II})$ metalated Pp fragments, which are linked through triangular $\mathrm{Cd}\left(\mathrm{CO}_{2}\right)_{3}$ fragments (Fig. 27c and d). It is important that MMPF-5 preserves its single crystalline character after the metal ion exchanges with $\mathrm{Co}(\mathrm{II})$.

Post-synthetic incorporation of $\mathrm{Zn}$ into free-base of $\mathrm{Al}(\mathrm{III})$ MOF with formation of light-harvesting MOFs proceeds by similar way. ${ }^{63}$ Reaction of MOF with anhydrous zinc acetate in DMF at $100{ }^{\circ} \mathrm{C}$ afforded a highly crystalline purple material, consistent with $90 \%$ occupancy of the Pp center sites by $\mathrm{Zn}$ (II). Visible light catalytic activity of this Zn-Pp based material is shown on sacrificial segregation of hydrogen from water.
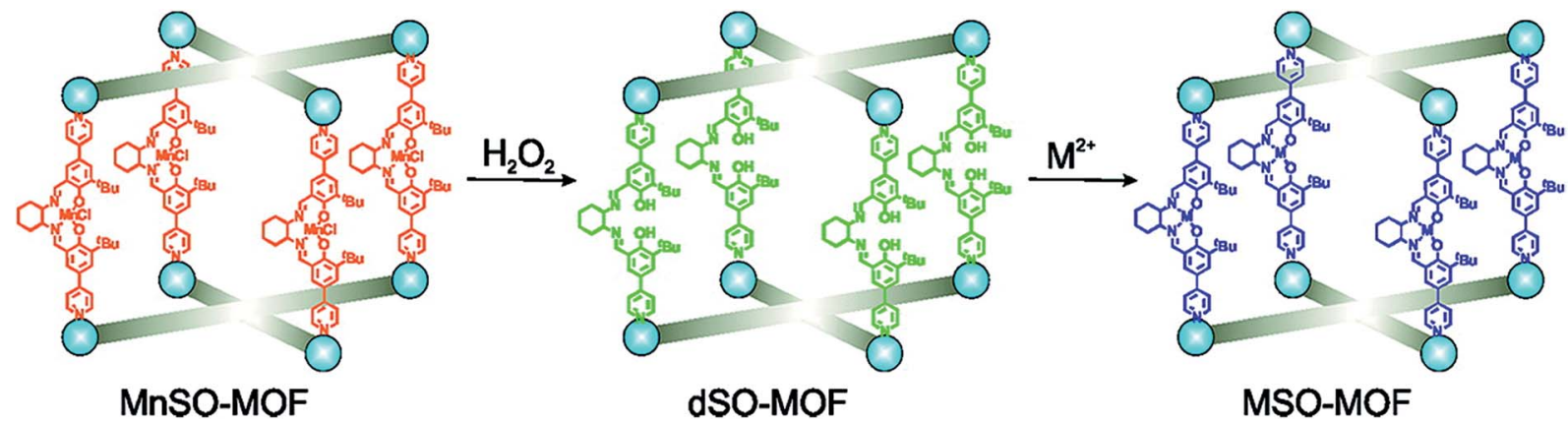

Scheme 23 Demetalation of Mn(III)SO-MOF and its subsequent remetalation. [Reprinted with permission from ref. 285. Copyright 2011, American Chemical Society.] 

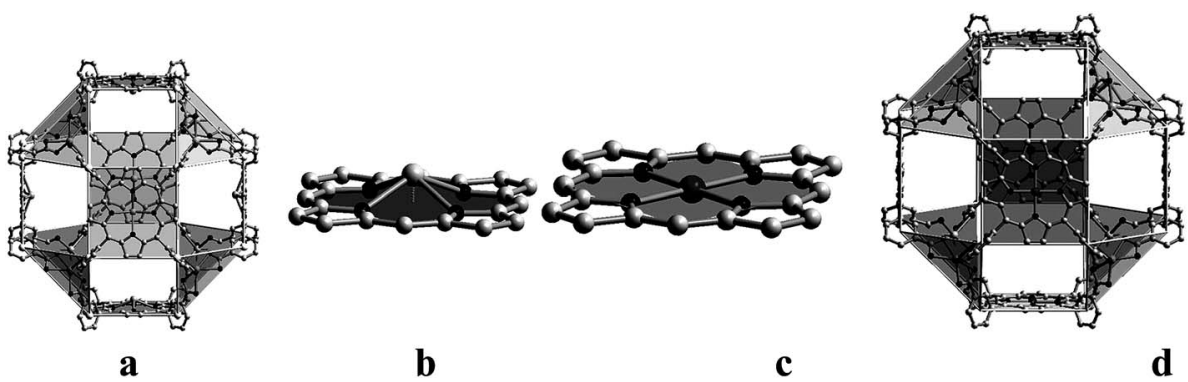

Fig. 27 (a) Representation of the crystal structure of the small cubicuboctahedron in MMPF-5 formed by 6 square Cd-Pp moieties and 8 triangular $\mathrm{Cd}\left(\mathrm{CO}_{2}\right)_{3}$ moieties. (b) The M-Pp macrocycle in MMPF-5, showing that the $\mathrm{Cd}(\mathrm{II})$ cation lies far out of the Pp plane. (c) The M-Pp macrocycle in MMPF-5(Co), showing that Co(॥) cation is located within the plane of the Pp. (d) The small cubicuboctahedron in MMPF-5(Co) formed by 6 square Co-Pp moieties and 8 triangular $\mathrm{Cd}\left(\mathrm{CO}_{2}\right)_{3}$ moieties. [Reprinted with permission from ref. 60. Copyright 2013, Wiley-VCH Verlag GmbH \& Co. KGaA, Weinheim.]

Of interest is post-synthetic metalation of reliable chiral and porous BINAP-based Zr-MOFs UiO topologies with $\mathrm{Rh}(\mathrm{nbd})_{2} \mathrm{BF}_{4}$ and $[\mathrm{Rh}(\mathrm{nbd}) \mathrm{Cl}]_{2} / \mathrm{AgSbF}_{6}$, where nbd is norbornadiene, to give highly active enantioselective one-site solid catalysts for reaction of asymmetric cyclization of 1,6-enynes. ${ }^{289}$

Extremely flexible and strong Cd-MOF anion accessible with tetra-acid spacer, in particular, 3,3',5,5'-tetrakis( $p$-carboxyphenyl)-2,2',6,6'-tetramethoxy-1,1'-biphenyl, shows abundant post-synthetic metal exchange of metal sites with the metal ion set, which differs significantly by their charges, ion radius, and chemical origin. ${ }^{290}$ In particular, there are as many as 16 isostructural new MOFs with transition, lanthanide, and basic groups of metal ions.

Taking into account universality and practical usefulness of PSM and PSE for access to other difficult-to-synthesize MOFs as bulk materials, their application to MOFs in thin film can also be useful. Among the most universal and useful approaches to synthesis of MOF films is layer-by-layer (LbL) coordination, which is called liquid phase epitaxy. ${ }^{50,291-294}$ LbL approach successfully and repetitively inputs solutions of each building (a)

\section{SALEF-1}

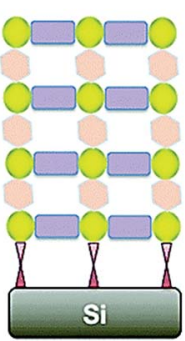

(b) $f=$ 3-APTMS

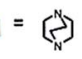

L2-MOF

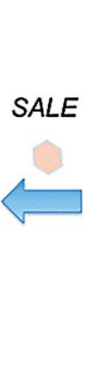

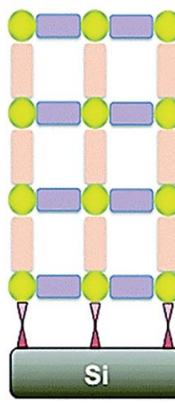

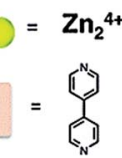

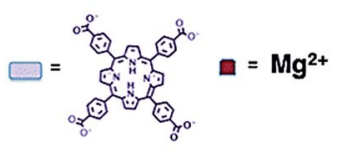

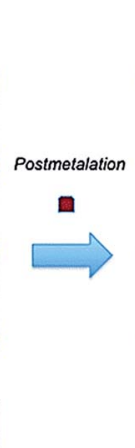

Scheme 24 SALE and post-synthetic metalation of MOF films. [Reproduced from ref. 297 with permission from The Royal Society of Chemistry.] block of a framework in contact with functionalized substrates, and then a MOF film grows. It is important that composition of additional layer not necessarily coincides with previous layers. ${ }^{295,296}$ Reliability of LbL-assembled pillared-paddlewheel MOF films in direction of transformation into new or modified MOFs through SALE and post-synthetic metalation has been demonstrated. ${ }^{297,298}$ In particular, it is shown that LbL synthesis provides MOFs, which are unavailable through other de novo strategies (Scheme 24). MOF contains 5,10,15,20-(4carboxyphenyl)-Pp units as tetratopic spacers, which coordinate pairs of $\mathrm{Zn}$ (II) ions in paddlewheel mode, and 4,4'-bipy spacer, which work as $\mathrm{Zn}$ (II)-ligating spacers or pillars between Pp defined layers. A dabco was used as a spacer for exchange or replacement of 4,4'-bipy pillars MOF. In addition, after $12 \mathrm{~h}$ reaction in excess magnesium bromide diethyl etherate, the free-base Pp containing films grown of quartz slides were transformed into M-Pp form.

Nevertheless, there are some drawbacks of post-synthetic approaches. For example, it is difficult to solve spatial distribution of functional groups post-synthetically included in MOF. As a result, most MOF structures are hypothetical. In some cases, a degree of post-synthetic incorporation of functional groups in MOFs is very limited. Moreover, further studies should investigate chemical principles of their specific phenomena, including SALE and transmetalation in order to improving the direct synthesis of MOFs with required chelating functionality.

\section{Thermolysis of coordination polymers as a route to nanostructures materials}

Recently CPs have become widely used as templates for a variety of nanomaterials. ${ }^{52,298}$ Unlike other templates, they produce monodisperse nanoparticles of high quality, while maintaining the porosity and morphology of the template precursor. Especially CPs widely used for the preparation of metal, metal oxide and carbon nanomaterials.

As a typical example, we note the strategy of using MOF thermolysis for the preparation of metal and metal oxides nano- 


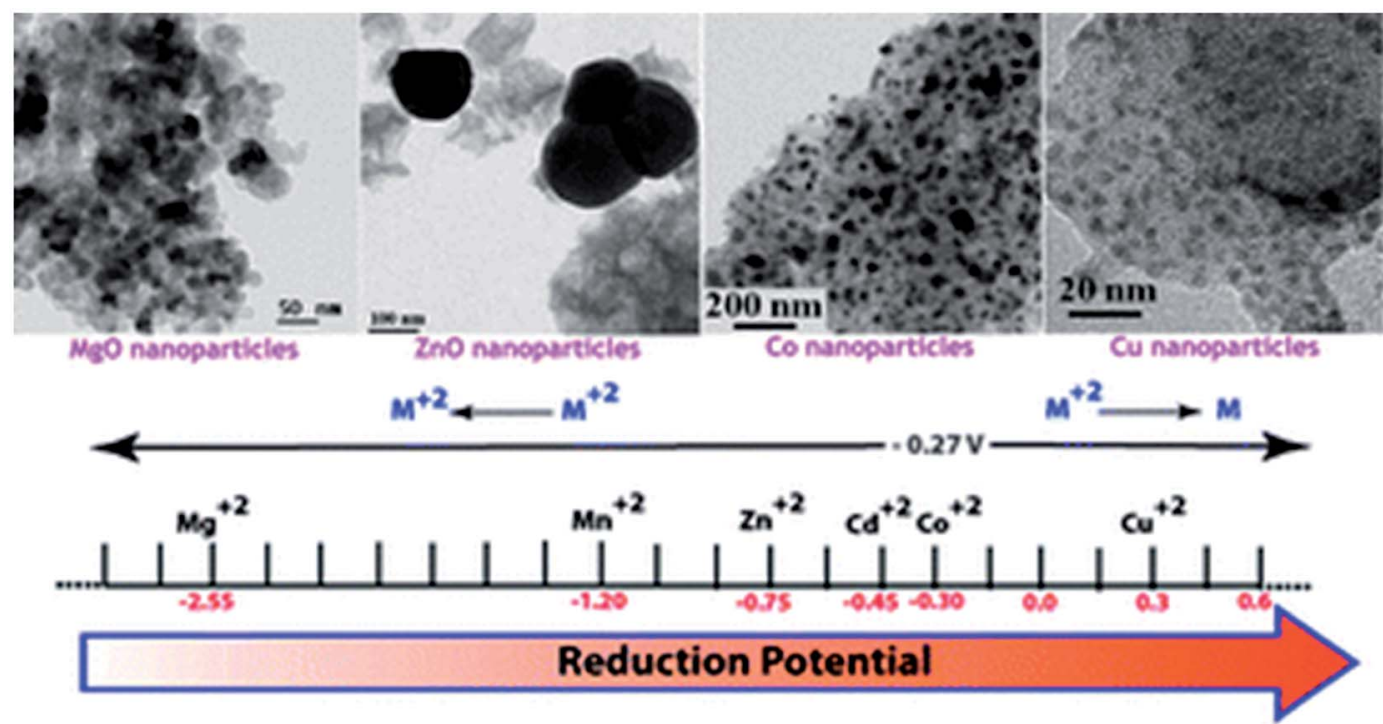

Fig. 28 Relation between the formed nanoparticle size and reduction potential. [Reproduced from ref. 299 with permission from The Royal Society of Chemistry.]

sized particles, such as $\mathrm{Cu} / \mathrm{CuO}, \mathrm{Co} / \mathrm{Co}_{3} \mathrm{O}_{4}, \mathrm{ZnO}, \mathrm{Mn}_{2} \mathrm{O}_{3}, \mathrm{MgO}$ and $\mathrm{CdS} / \mathrm{CdO} .{ }^{299}$ According to this strategy, during thermolysis in $\mathrm{N}_{2}$, metals with a reduction potential of $-0.27 \mathrm{~V}$ or higher contained in MOFs always form pure metal nano-sized particles, whereas metals with a reduction potential lower than $-0.27 \mathrm{~V}$ form metal oxide nano-sized particles (Fig. 28). It is important that there is a relationship between the size of the formed nanoparticle and the distance between the SBU inside the MOF precursors. In addition, the crystallinity of the carbon matrix was also influenced by the thermolysis conditions $\left(\mathrm{N}_{2}\right.$ and air).

\subsection{Synthesis of carbon materials}

Interest in various forms and allotropic modifications of carbon is very wide from use as adsorbents, carriers for catalysts and drug delivery to the electrode materials. The highly porous carbon materials may be prepared by various methods, including physical and chemical activation of the carbon, polymer aerogels carbonization and templating methods using zeolites and mesoporous silica. ${ }^{300}$ Often, however, obtained mesoporous carbon materials are characterized by a broad polydispersity to micron-sized particle and their fuzzy shape.

The direct thermal transformation of CPs was used for the preparation of porous carbons with special morphologies (for example, cube and polyhedron etc.), ${ }^{301-303}$ and their pore structures may be easily varied depending on the as-formed metal or metal oxide nano-sized particles. However, using thermolysis of CPs for the preparation of nanocomposites is restrained by their high cost. ${ }^{202,304}$

Aluminium-diethylenetriamine pentaacetic acid (Al-DTPA) microfibers were used as cost-effective precursors (Fig. 29) to prepare nitrogen-doped carbon microfibers (NCF).$^{305}$

In particular, thermal transformation and acid-leaching of Al-DTPA leads to the well-defined NCFs. It is important to underline that the microfiber morphology of Al-DTPA persists in the resulting carbon materials (Fig. 30B-E). The typical NCFs prepared at $900{ }^{\circ} \mathrm{C}$ (NCF-900) have the diameters of $\sim 500 \mathrm{~nm}$ and lengths of about $50 \mathrm{~mm}$ (Fig. 30B-D). It should be noted that some graphitization regions are observed in the highresolution TEM image of NCF-900 (Fig. 30E). Increasing thermolysis temperature leads to a slight increase in the graphitization degree of carbon microfibers (Fig. 30E inset). Moreover, considerable interconnected pores are homogeneously distributed in the whole microfibers (Fig. 30D). The pore size distribution curves exhibited the mesopores with

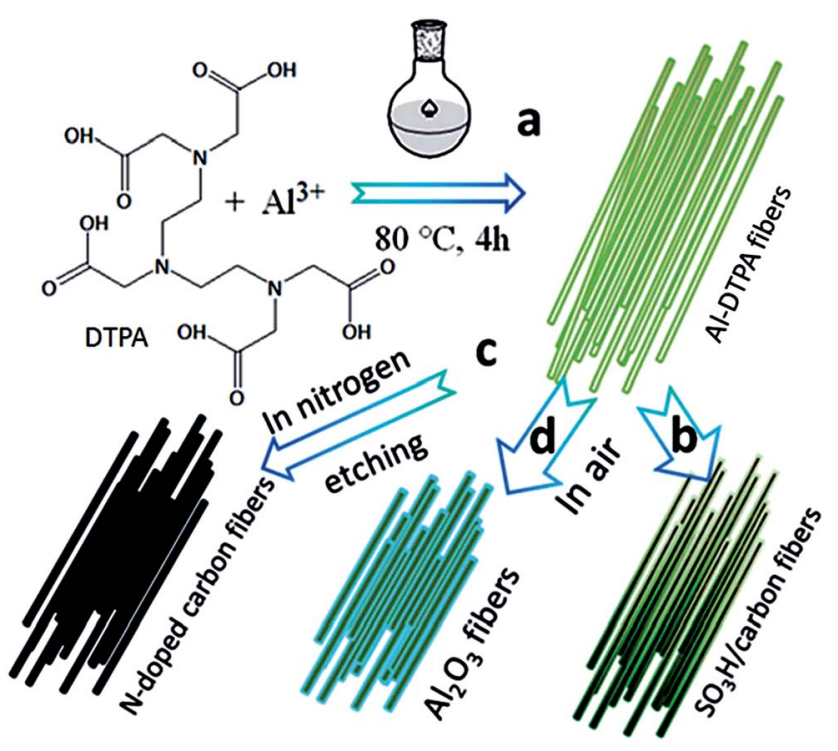

Fig. 29 Schematic illustration of the formation process of the nanoporous $\mathrm{N}$-doped carbon microfibres and solid acids microfibers. [Reproduced from ref. 305 with permission from The Royal Society of Chemistry.] 


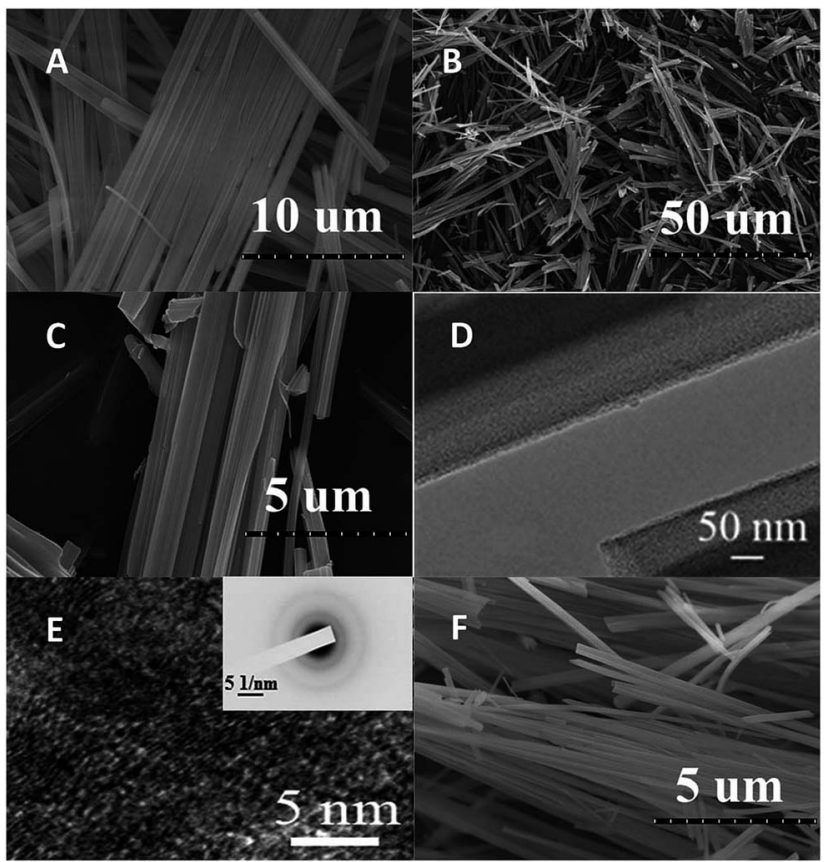

Fig. 30 (A) SEM images of Al-DTPA microfibers; (B and C) SEM images of NCF-900; ( $D$ and E) TEM images of NCF-900. Inset is the corresponding selected area electron diffraction pattern of NCF-900; (F) SEM images of $\gamma-\mathrm{Al}_{2} \mathrm{O}_{3}$ obtained by heating Al-DTPA at $800^{\circ} \mathrm{C}$ in air. [Reproduced from ref. 305 with permission from The Royal Society of Chemistry.]

sizes of 2-30 nm. The total BET surface area of NCF-750, -900, and -1000 are equal to 604,933 and $1072 \mathrm{~m}^{2} \mathrm{~g}^{-1}$, respectively. Thus, the NCFs with nanoporous structure, uniform fiber morphology, and appropriate degree of graphitization were successfully obtained by thermolysis of Al-DTPA. It should be noted that Al-DTPA microfibers can be also used as the precursors of $\gamma-\mathrm{Al}_{2} \mathrm{O}_{3}$ microfibers. After thermolysis of Al-DTPA in air at $800{ }^{\circ} \mathrm{C}$, the well-defined microfiber morphology in $\gamma-\mathrm{Al}_{2} \mathrm{O}_{3}$ was observed in SEM images (Fig. 30F). As compared to Al-DTPA microfibers, there is decreasing the diameter $(350 \mathrm{~nm})$ and length $(50 \mathrm{~mm})$ (Fig. 30A). The prepared $\gamma-\mathrm{Al}_{2} \mathrm{O}_{3}$ has a smaller BET surface area $\left(50 \mathrm{~m}^{2} \mathrm{~g}^{-1}\right)$ and pore size distribution in the range of 2-15 $\mathrm{nm}$.

Various types of porous carbon materials may be prepared in a simple manner, based on the MOF structures as template molecules and a carbon source. Due to the high thermal stability, the nanoporous structure and the possibility to penetrate into the pores of small molecules with their subsequent participation in different 《/ship-in-bottle $\rangle$ reactions, MOFs can be considered as rigid templates similar to the mesoporous silica gel and zeolites. Given the wide variety of MOFs of different compositions and morphology, the carbon materials can be produced with controlled pore texture and surface area. Moreover, the synthetic strategy based on MOFs allows to producing various carbon materials with catalytic and magnetic properties. ${ }^{306}$

Advantages of MOFs templates can be realized very efficiently in the production of various composite carbon

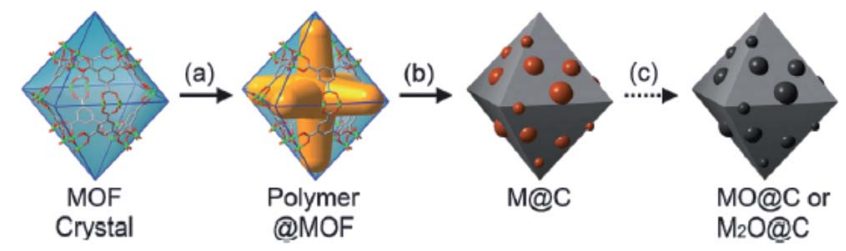

Fig. 31 Scheme of the transformation of MOFs in M/MO@C composite. [Reproduced from ref. 307 with permission from The Royal Society of Chemistry.]

materials, for example, type M/MO@C (Fig. 31). The general approach of the synthesis is based on the fact that the $1^{\text {st }}$ stage of polymerization is carried out in MOFs carbon precursor (Fig. 31a), then a M@C nanocomposite is formed by thermolysis (Fig. 31b), and finally post-thermal treatment is carried out to convert the metal to metal oxide nanoparticles (Fig. 31c). The porosity of the carbon matrix and M/MO nanoparticle sizes are controlled in stages. ${ }^{307}$

It should be noted the obtaining a porous cobalt oxidecarbon hybrid by carbonizing in an inert atmosphere and subsequently air-calcining nanocrystals of ZIF-67. ${ }^{308}$ It is important that the synthesized carbon composite material can act as an efficient electrochemical water oxidation catalyst.

\subsection{The preparation of metal oxide materials}

As a rule, the basis of the methods for producing metal oxide nanocomposites from $\mathrm{CP}$ is a single-stage solid-phase thermolysis of the precursor source, as, in general, quite comfortable, well-reproducible and easily scalable process. The methodology was used in the synthesis of nanoparticles of various metal oxides, for example, $\mathrm{PbO},{ }^{309} \mathrm{ZnO},{ }^{310} \mathrm{Fe}_{2} \mathrm{O}_{3}{ }^{311}$ $\mathrm{Co}_{3} \mathrm{O}_{4},{ }^{312} \mathrm{CdO},{ }^{52} \mathrm{CuO} / \mathrm{Cu}_{2} \mathrm{O},{ }^{313,314} \mathrm{Tl}_{2} \mathrm{O}_{3},{ }^{315}$ etc. We consider some of the most common examples.

Thus, $\left[\mathrm{Pb}_{2}(2,9-\text { dimethyl-phen })_{2}\left(\mu-\mathrm{N}_{3}\right)_{2}\left(\mu-\mathrm{ClO}_{4}\right)_{2}\right]_{n}$ (ref. 316) and [Cd(2,9-dimethyl-phen)(1-(2-thenoyl)-3,3,3-trifluoroacetonate) $\left.)_{2}\right]_{n}$ (ref. 317) were used for $\mathrm{PbO}$ and $\mathrm{CdO}$ nanoparticles preparation, respectively. Thermal decomposition of the $3 \mathrm{D} \mathrm{CP}[\mathrm{Pb}($ phen)(1$\left.\left.\mathrm{N}_{3}\right)\left(1-\mathrm{NO}_{3}\right)\right]_{n}$ containing an azide-anion ligand gave $\mathrm{PbO}$ nanoparticles. ${ }^{318}$

$\mathrm{ZnO}$ nanomaterials with desired morphologies have been obtained by thermal decomposition of appropriate CPs and MOF precursors. ${ }^{52,309,319}$ For example, calcination of infinite rectangular-tubular helices $\left[\mathrm{ZnCl}_{2} \mathrm{~L}\right]$ and $\left[\mathrm{ZnBr}_{2} \mathrm{~L}\right]$ (L is bis(3pyridyl)cyclotetramethylenesilane) at $500{ }^{\circ} \mathrm{C}$ gives uniform hexagonal tubular spire crystals of $1.2 \times 1.2 \times 4.0 \mu^{3}$ dimensions and spheres, respectively. ${ }^{320}$ SEM images of the products of $\left[\mathrm{MX}_{2} \mathrm{~L}\right]$ thermolysis at $500{ }^{\circ} \mathrm{C}$ (Fig. 32) show that the compounds ultimately change to zinc(II) oxide crystals. For $\left[\mathrm{ZnCl}_{2} \mathrm{~L}\right]$, calcination at $500{ }^{\circ} \mathrm{C}$ leads to the growth of each $\mathrm{ZnO}$ single crystal.

The Zn(II) oxide and Cd(II) oxide nanoparticles were prepared by thermolysis of $\mathrm{Zn}(\mathrm{II})$ and $\mathrm{Cd}(\mathrm{II}) \mathrm{CPs},\left[\mathrm{Zn}(\mathrm{L})_{2}\left(\mathrm{~N}_{3}\right)_{2}\right]_{n},\left[\mathrm{Zn}(\mathrm{L})_{2}{ }^{-}\right.$ $\left.(\mathrm{SCN})_{2}\right]_{n},\left[\mathrm{Cd}(\mathrm{L})_{2}\left(\mathrm{~N}_{3}\right)_{2}\right]_{n}$ and $\left[\mathrm{Cd}(\mathrm{L})_{2}(\mathrm{SCN})_{2}\right]_{n}$, where $\mathrm{L}$ is 2 -aminomethylpyridine. ${ }^{321}$ SEM images exhibit the average size of prepared $\mathrm{ZnO}$ and $\mathrm{CdO}$ nanoparticles of 60-70 $\mathrm{nm}$. 

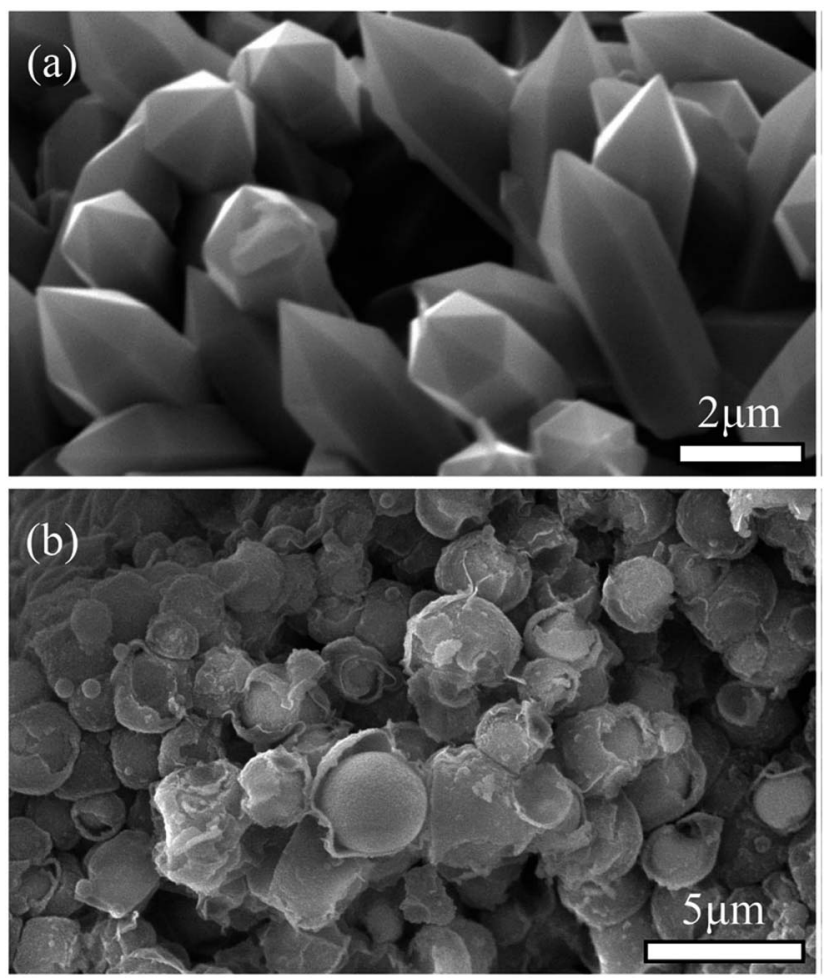

Fig. 32 SEM images of thermal decomposition residue of $\left[\mathrm{ZnCl}_{2} \mathrm{~L}\right](\mathrm{a})$ and $\left[\mathrm{ZnBr}_{2} \mathrm{~L}\right]$ (b) calcined at $500^{\circ} \mathrm{C}$ for $2 \mathrm{~h}$. [Reproduced from ref. 320 with permission from The Royal Society of Chemistry.]

The calcinations of nanoparticles of a $3 \mathrm{D} \mathrm{Cd(II)} \mathrm{compound,}$ $\left[\mathrm{Cd}(\mathrm{L})_{2}\left(\mathrm{H}_{2} \mathrm{O}\right)_{2}\right]\left(\mathrm{L}^{-}\right.$is $1 \mathrm{H}$-1,2,4-triazole-3-carboxylate), at $650{ }^{\circ} \mathrm{C}$ under air yielded $\mathrm{CdO}$ nanoparticles. ${ }^{322}$

A series of lead-containing CPs have been applied for $\mathrm{PbO}$ nanoparticle formation. $\mathrm{PbO}$ nanoparticles serve as catalyst for multicomponent organic synthesis reactions, for instance PaalKnorr reaction and oxidative coupling of methane, due to the significant catalytic property with operational simplicity, high reactivity, environmental friendliness, reduction of reaction times, and reusability of $\mathrm{PbO}$ nanoparticles. ${ }^{323}$ In this respect, a CP of lead(II) nitrate complexes with bridging trans-1,2-bis(4-pyridyl) ethene (L) ligand, $\left[\mathrm{Pb}\left(\mu-\mathrm{NO}_{3}\right)_{2}(\mathrm{~L})(\mathrm{MeOH})\right]_{n}$, was used $^{324}$ to prepare $\mathrm{PbO}$ nanoparticles via two different methods.

The nanostructure of $1 \mathrm{D}$ copper $\mathrm{CP},\left[\mathrm{Cu}(\mathrm{HL}) \mathrm{NO}_{3}\right]_{n}\left(\mathrm{H}_{2} \mathrm{~L}\right.$ is $[2-$ [1-(2-hydroxypropylimino)ethyl] phenol]), was used as precursor to obtain single phase $\mathrm{CuO}$ nano-sized particles by thermolysis. ${ }^{325}$ According to the SEM images the diameters of the prepared products were equal to $40 \mathrm{~nm}$ (Fig. 33).

Calcinations of the nano-sized copper(II) CP, $\left[\mathrm{Cu}(\mathrm{L})\left(\mathrm{H}_{2} \mathrm{O}\right)_{2}\right]_{n}$ $[\mathrm{L}=2,6$-pyridinedicarboxylate $]$, at $500{ }^{\circ} \mathrm{C}$ under air yielded $\mathrm{CuO}$ nanoparticles. $^{326}$

After calcining the cerium-based CPs with three isomers of benzenedicarboxylic acid at high temperature, micro/ nanostructures of ceria such as nanoparticles, nanorods, and microflowers with retained morphologies were achieved. ${ }^{327}$

Thermolysis of the porous CPs with nanorod morphology based on a catechol-substituted Pp [meso-tetrakis(3,4-dihydroxyphenyl)-

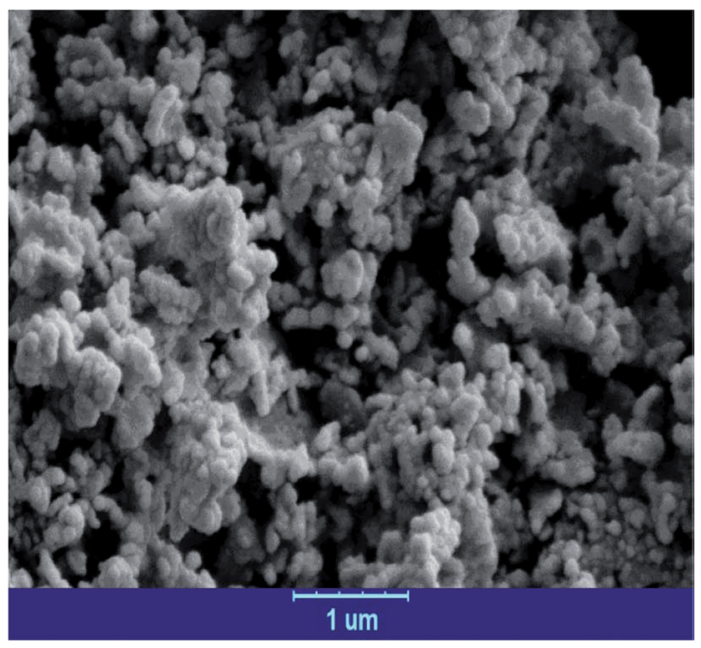

Fig. 33 SEM images of $\mathrm{CUO}$ nanoparticles prepared by direct calcination of $\mathrm{Cu}(\mathrm{II}) \mathrm{CP}$. [Reprinted with permission from ref. 325. Copyright 2016, Taylor \& Francis.]

Pp] led to carboniferous materials containing metal oxide nanoparticles with large surface areas (up to $800 \mathrm{~m}^{2} \mathrm{~g}^{-1}$ ). ${ }^{328}$

The advantages of these CP precursors are simplicity of processing and long-range ordering that allow the synthesis of metal oxides with various morphologies. Phase composition and properties of the resulting metal oxide nanocomposites depend on the crystal structure and morphology of the starting $\mathrm{CP}$ precursor, its particle size, and the synthesis conditions (the influence of the temperature, environment, the nature of the surfactant and etc.).

5.2.1. Influence of the original morphology and particle size of coordination polymers. Size and morphology of the produced nanomaterials are highly dependent on the type and morphology of the CP precursor. ${ }^{52,329-331}$

Porous $\gamma-\mathrm{Fe}_{2} \mathrm{O}_{3}$ nanoparticles were prepared via a solid-state transformation process of a mesoporous iron(III) carboxylate crystal, MIL-100(Fe). ${ }^{332}$ The $\mathrm{N}_{2}$ adsorption-desorption analysis also demonstrates the mesoporous character of the derived $\gamma$ $\mathrm{Fe}_{2} \mathrm{O}_{3}$ material, with type IV $\mathrm{N}_{2}$ isotherm at $77 \mathrm{~K}$. This material has a relatively large specific surface area $123.5 \mathrm{~m}^{2} \mathrm{~g}^{-1}$, which is presumed to be benefited from the two-step calcination of the template MIL-100(Fe). On the other hand, the pore size distribution reveals that most of the pores are focused on $10 \mathrm{~nm}$, indicating the mesopores are effective formed for the $\gamma-\mathrm{Fe}_{2} \mathrm{O}_{3}$.

Of interest is using MOF thermolysis to develop the porous iron oxides. ${ }^{33-336}$ As an example, we note the fabrication of spindle-like mesoporous $\alpha-\mathrm{Fe}_{2} \mathrm{O}_{3}$ using MOF MIL-88(Fe) as template. ${ }^{310}$ The prepared spindle-like mesoporous $\alpha-\mathrm{Fe}_{2} \mathrm{O}_{3}$ was composed of clustered $\mathrm{Fe}_{2} \mathrm{O}_{3}$ nanoparticles with size of $<20 \mathrm{~nm}$. In another example, a facile synthesis of porous $\mathrm{Fe}_{2} \mathrm{O}_{3}$ nanocubes by simultaneous oxidative decomposition of Prussian blue nanocubes was demonstrated. ${ }^{337,338}$ The derived porous $\mathrm{Fe}_{2} \mathrm{O}_{3}$ nanocubes are composed of very fine $\mathrm{Fe}_{2} \mathrm{O}_{3}$ nanoparticles with size of several nanometers. It is important that MOFs show distinct advantages due to their well-ordered crystalline 
structure, high porosity, large surface area, and tunable pore size, in comparison with other templates.

It should be noted that during the MOF pyrolysis it can also be prepared metal oxide nanostructure consisting of the same primary particles, but with a different secondary and tertiary architecture thus modulating the macroscopic structure of the initial CP. For example, two types of $\mathrm{Co}_{3} \mathrm{O}_{4}$ nanomaterials with plate-like and rod-like morphology of the nanoparticles, based on the Co-containing plate-shaped $\left(\left[\mathrm{Co}_{3}(\mathrm{~L})_{3}(\mathrm{DMF})_{4}\right]_{n}, \mathrm{p}-\mathrm{MOF}\right)$ and rod-shaped $\left([\mathrm{Co}(\mathrm{L})(\mathrm{DMSO})]_{n}\right.$, r-MOF) CPs, consisting of the same composite units $\left(\mathrm{Co}^{2+}\right.$ and 1,4-benzenedicarboxylate (L)), were prepared through pseudomorphic transformation. ${ }^{338}$

Thermolysis of nano plates of two Cd(II)-based MOFs, $\left[\mathrm{Cd}_{2}(\mathrm{~L})_{2}\left(\mathrm{~L}^{\prime}\right)_{2}\right]_{n} \cdot(\mathrm{DMF})_{x}(\mathrm{TMU}-8)$ and $\left[\mathrm{Cd}(\mathrm{L})\left(4,4^{\prime}-\mathrm{bipy}\right)\right]_{n} \cdot(\mathrm{DMF})_{y}$ (TMU-9) where $\mathrm{H}_{2} \mathrm{~L}$ is $\mathrm{V}$-shaped flexible dicarboxylate ligand $4,4^{\prime}$-oxybis(benzoic acid) and the $\mathrm{L}^{\prime}$ is $\mathrm{N}$-donor ligand 1,4-bis(4pyridyl)-2,3-diaza-1,3-butadiene, leads to CdO nano-sized particles (Fig. 34). ${ }^{339}$ SEM images of the products exhibit the formation of aggregation of $\mathrm{CdO}$ nano-sized particle in the range of $60-160 \mathrm{~nm}$. Particle sizes of CdO prepared by thermolysis of TMU-9 are smaller than those prepared from calcination of TMU-8 that is associated with the influence of the structures of these two MOFs on the final morphologies of CdO particles. ${ }^{52}$

The 1D CP, $\left[\mathrm{Zn}\left(4,4^{\prime}\right.\right.$-bpy $\left.)\left(\mathrm{H}_{2} \mathrm{O}\right)_{4}\right](\mathrm{L}) \cdot 4 \mathrm{H}_{2} \mathrm{O}\left(\mathrm{H}_{2} \mathrm{~L}\right.$ is acetylenedicarboxylic acid), and $3 \mathrm{D} \mathrm{MOF}, \mathrm{Zn}(\mathrm{L})_{2} \cdot\left(\mathrm{L}^{\prime}\right)_{2}\left(\mathrm{~L}^{\prime}\right.$ is triethylamine) were applied as precursors for the synthesis of $\mathrm{ZnO}$ nanocrystals via direct thermal decomposition. ${ }^{340}$ Interestingly, changing the precursor from 1D CP to 3D MOF changes the morphology from nanorods to nanoparticles.

Thermolysis of the bulk powder and nano-sized Ni(II) MOF with cubic building units and $1 \mathrm{D}$ open channels, i.e. $\left\{\left[\mathrm{Na}_{16^{-}}\right.\right.$ $\left.\left.\left(\mathrm{Ni}_{8} \mathrm{~L}_{12}\right)\left(\mathrm{H}_{2} \mathrm{O}\right)_{20}\left(\mathrm{H}_{2} \mathrm{O}\right)_{4}\right]\left(\mathrm{CH}_{3} \mathrm{CN}\right)\left(\mathrm{H}_{2} \mathrm{O}\right)_{18.5}\right\} \infty \quad\left(\mathrm{H}_{3} \mathrm{~L}=4,5-\right.$ imidazoledicarboxylic acid), at $700{ }^{\circ} \mathrm{C}$ under air yielded $\mathrm{NiO}$ nanoparticles, which size and morphology depends on the particle size of the initial compound. ${ }^{341}$ The crystalline and nano-structures of two $\mathrm{Pb}(\mathrm{II}) \mathrm{CPs},\left[\mathrm{Pb}_{2}(\mathrm{~L})_{4}(\mathrm{MeOH})\right]_{n}(\mathrm{~L}=2$-pyrazinecarboxylic acid) and $\left[\mathrm{Pb}\left(\mathrm{L}^{\prime}\right)_{2}\right]_{n}\left(\mathrm{~L}^{\prime}=2\right.$-quinolinecarboxylic acid), were thermolyzed to prepare $\mathrm{PbO}$ nanoparticles with various morphology and size. ${ }^{254}$

It should be noted that N,S-containing ligands were used not only to prepare metal sulfide nanoparticles, as expected, but also metal oxides, more thermodynamically stable in air. Thus, a ZnO nanostructure was prepared by thermolysis of nanoparticles of a $\mathrm{Zn}(\mathrm{II}) \mathrm{CP},\left[\mathrm{Zn}(\mathrm{L})\left(\mathrm{CH}_{3} \mathrm{COO}\right)_{2}\right]_{n}\left(\mathrm{~L}=2,2^{\prime}\right.$-diamino$5,5^{\prime}$-dimethyl-4, $4^{\prime}$-bithiazole), at $500{ }^{\circ} \mathrm{C}$ under air. ${ }^{342}$

Saving macroscopic MOFs structure during their thermal transformation may serve as a promising strategy in the design of nanomaterials with predetermined properties and morphology.

5.2.2. Influence of temperature. Temperature is an important parameter controlling the phase composition and morphology of the resulting nanostructures. Thus, controlled thermolysis of $\left[\mathrm{Mn}_{2}(\mathrm{~L})(\mathrm{DMA})_{2}\right]_{n}$ ( $\mathrm{L}$ is meso-butane-1,2,3,4tetracarboxylate, DMA is $N, N$-dimethylacetamide $)^{343}$ and $\mathrm{Mn}_{2}\left(\mathrm{~L}^{\prime}\right)$ ( $\mathrm{L}^{\prime}$ is 2,5-dihydroxyterephthalate $)^{344}$ by varying the temperature and the atmosphere leads to the formation of a series of nanoporous manganese oxides with different degrees of oxidation: $\mathrm{MnO}, \mathrm{Mn}_{3} \mathrm{O}_{4}, \mathrm{Mn}_{5} \mathrm{O}_{8}$, and $\mathrm{Mn}_{2} \mathrm{O}$ and preserving the original morphology of the starting precursor (Fig. 35). In addition, varying the thermolysis temperature $\left(300-700{ }^{\circ} \mathrm{C}\right)$ of $\mathrm{Mn}_{2}\left(\mathrm{~L}^{\prime}\right)^{344}$ leads to reduction of lattice defects, increasing the crystallinity degree of the thermolysis products. At the same time, the crystal surface becomes uneven and a plurality of voids appears with temperature increasing. The prepared $\mathrm{MnO}_{x}$ are characterized by type IV adsorption isotherms, indicating their mesoporous structure. It should be noted that in this way
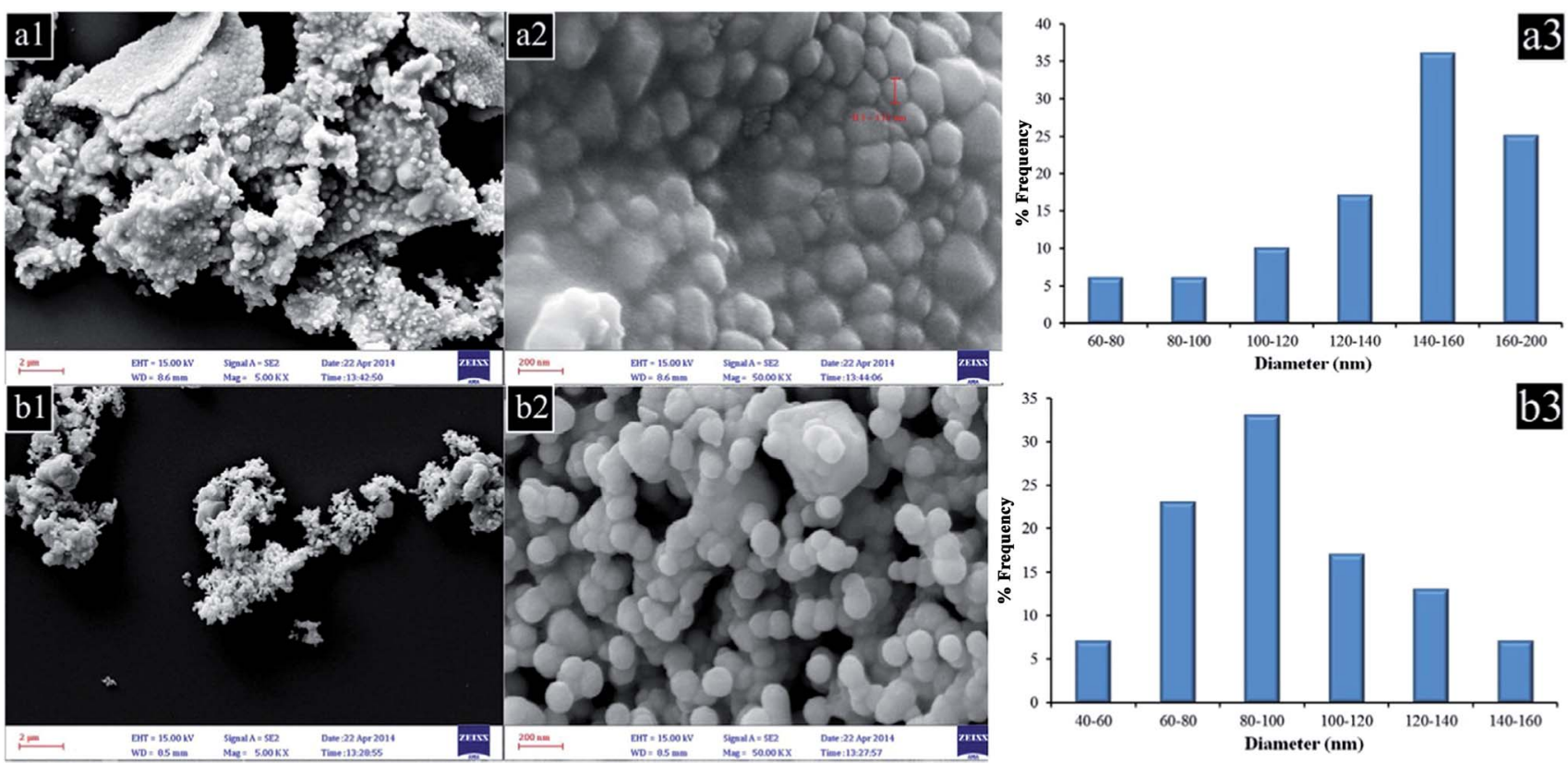

Fig. 34 FE-SEM images and the corresponding particle size distribution histogram of CdO nanoparticles prepared by thermolysis of (a) TMU-8 and (b) TMU-9 at $550{ }^{\circ} \mathrm{C}$. [Reprinted with permission from ref. 339. Copyright 2016, Elsevier.] 

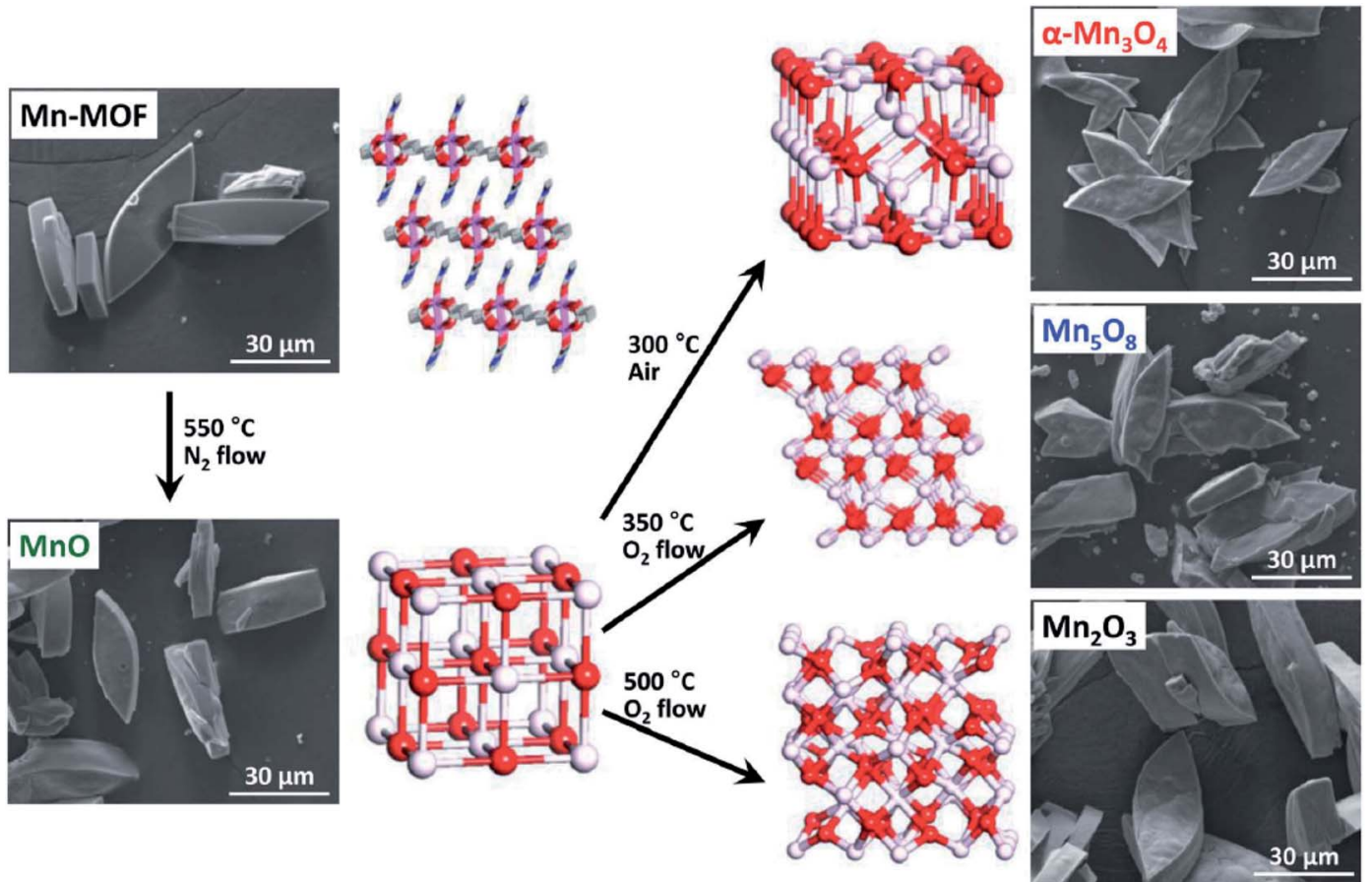

Fig. 35 SEM micrographs and diagram of structural transformations of $\left[\mathrm{Mn}_{2}(\mathrm{~L})(\mathrm{DMA})_{2}\right]_{n}$ in manganese oxides during thermolysis. $[$ Reproduced from ref. 343 with permission from The Royal Society of Chemistry.]

rare low-valence oxides $\mathrm{MnO}$ and metastable phases of $\mathrm{Mn}_{5} \mathrm{O}_{8}$ with nanoporous architecture can be obtained, i.e. MOF thermal transformation can significantly enrich the composition, structure and functional properties of the resulting nanoporous metal oxide materials. ${ }^{345}$

Similar patterns are characteristic of thermal transformations of MIL-100(Fe). ${ }^{346}$ The average size of nanorods $\mathrm{Fe}_{2} \mathrm{O}_{3}$, obtained at temperatures of 350,550 and $750^{\circ} \mathrm{C}$ is 50 , 150 and $200 \mathrm{~nm}$, respectively. Character of adsorptiondesorption isotherms in this case is consistent with the general trend of the formation of the mesoporous structure of metal oxide nanocomposites obtained by MOF thermolysis. As a rule, surface area, pore volume, and pore size decreases with increasing thermolysis temperature, which also is in agreement with the fact that the thermally controlled crystal growth reduces the porosity with increasing process temperature.

Thermolysis temperatures of two silver(I) CPs, $[\mathrm{Ag}(\mathrm{HQ})(\mathrm{Q})]_{n}$ and $\left\{\left[\mathrm{Ag}(\mathrm{HQ})_{2}\right] \mathrm{NO}_{3}\right\}_{n}$, has effect on the formation of silver nanomaterials. ${ }^{347}$ In particular, increasing temperature leads to increase of agglomeration of silver nanoparticles. Calcination of sinusoidal $\mathrm{CP}[\mathrm{Ag}(\mathrm{L})]\left(\mathrm{ClO}_{4}\right)$, where $\mathrm{L}$ is a diethylbis(4-pyridyl) silane, at $400{ }^{\circ} \mathrm{C}$ gives circle morphology with evolving burned organics. At the same time, at $600{ }^{\circ} \mathrm{C}$, network circles involving of a silver(0)/silver chloride (chlorargyrite)/silicon(rv) oxide composite with a micro-sized convexo-concave surface are formed. ${ }^{348}$ However, thermolysis of $[\mathrm{Ag}(\mathrm{L})]\left(\mathrm{BF}_{4}\right)$ crystals at $600{ }^{\circ} \mathrm{C}$ gives silver(0) nanocomposites without silicon(Iv) oxide.

It should be noted the zinc(II) oxide nanoparticles prepared by thermolysis of $\left[\mathrm{Zn}(\mathrm{L})_{2}\left(\mathrm{H}_{2} \mathrm{O}\right)_{4}\right]_{n}, \quad\left[\mathrm{Zn}\left(\mathrm{L}^{\prime}\right)_{2}\left(\mathrm{H}_{2} \mathrm{O}\right)_{2}\right]_{n} \quad$ and
$\left[\mathrm{Zn}\left(\mathrm{H}_{2} \mathrm{O}\right)_{6}\right] \cdot\left(\mathrm{L}^{\prime \prime}\right)_{2}$, where $\mathrm{L}$ is nicotinic acid N-oxide, $\mathrm{L}^{\prime}$ is picolinic acid $\mathrm{N}$-oxide and $\mathrm{L}^{\prime \prime}$ is isonicotinic acid $\mathrm{N}$-oxide, at two temperatures (200 and $600{ }^{\circ} \mathrm{C}$ ) using two methods (with surfactant and without surfactant). ${ }^{349}$ Analysis of the SEM images of the prepared $\mathrm{ZnO}$ nanoparticles exhibits that small and spherical $\mathrm{ZnO}$ nanoparticles were prepared by thermolysis of compounds at $200{ }^{\circ} \mathrm{C}$ using surfactant (oleic acid).

5.2.3. Influence of surfactants. It should be noted using surfactants in the synthesis of nanomaterials to change the morphology and particle size. For example, Zn(II) CPs were used for the preparation of nano-sized materials in the presence of surfactants. ${ }^{350,351}$

Pure phase $\mathrm{ZnO}$ nanoparticles with various morphologies and sizes were synthesized by thermolysis of zinc(II) CPs, $[\mathrm{Zn}(\mathrm{L})(\mathrm{OAc})] \mathrm{ClO}_{4},\left[\mathrm{Zn}(\mathrm{L}) \mathrm{I}_{2}\right]$, and $\left[\mathrm{Zn}(\mathrm{L})_{2}\right]\left(\mathrm{ClO}_{4}\right)_{2}\left(\mathrm{H}_{2} \mathrm{O}\right)_{2.9}$ based on the same ligand $\mathrm{L}^{352}$

Of interest is the synthesis of $\mathrm{PbO}$ nanoparticles $(\sim 30 \mathrm{~nm})$ by thermolysis of a $\mathrm{Pb}(\mathrm{II})$ complex, $\left\{\left[\mathrm{Pb}_{2}(\mathrm{~L})_{2}\left(\mathrm{NO}_{3}\right)_{4}\right] \cdot \mathrm{MeOH}\right\}_{n}$, where $\mathrm{L}$ is $N, N^{\prime}, N^{\prime \prime}$-tris(pyrid-3-ylmethyl)-1,3,5-benzenetricarboxamide, at $180^{\circ} \mathrm{C}$ with oleic acid as a surfactant. ${ }^{353}$

We note also $\mathrm{PbO}$ nanoparticles $(\sim 25 \mathrm{~nm})$ obtained by thermolysis of plate-shaped nanostructures of a $1 \mathrm{D}$ lead(II) CP involving the $\mathrm{Pb}_{2}(\mu-\mathrm{I})_{2}$ fragment, $\left[\mathrm{Pb}(\mathrm{L}) \mathrm{I}_{2}\right]_{n}(\mathrm{~L}=$ neocuproine or 2,9-dimethyl-phen), with oleic acid as a surfactant. ${ }^{354}$

\subsection{Mixed-oxide nanocomposites}

Heterometallic chelates are of interest for preparation of functional oxide materials (e.g., metal-oxide ceramics). The main advantages of using heterometallic chelates as precursors of 
nanomaterials are low temperature of thermolysis, the exact ratio of metal ions, the formed nanoparticle size control, etc.

As a typical example, we consider using mixed lanthanide CPs for the production of rare earth oxides. ${ }^{355}$ In particular, welldefined cylindrical or spherical micro-morphologies can be prepared by varying solvents (water or THF) which were discovered in the resulting oxides after thermal treatment. Such bimetallic CPs were synthesized by the interaction of chlorides or nitrates of $\mathrm{Ce}, \mathrm{Nd}$, Gd with 2,5-dihydroxy-1,4-benzoquinone and used as precursors for mixed oxide ceramics. Thermolysis at $850{ }^{\circ} \mathrm{C}$ of the CPs leads to mixed $\mathrm{Nd}_{x} \mathrm{Gd}_{(1-x)}$ and $\mathrm{Nd}_{x} \mathrm{Ce}_{(1-x)}$ oxides as solid solutions, in which $x$ varies between 0 and 1 . It should be noted that using well-characterized CPs as precursors allows to controlling over the atom sequences in the chains, the number of coordination bonds and the dimensionality of the precursor structure.

The thermolysis of $\mathrm{CPs}\left[\mathrm{BaCu}(\mathrm{L})_{2}\right]_{n},\left[\mathrm{BaCu}(\mathrm{L})_{2}\left(\mathrm{H}_{2} \mathrm{O}\right)\right]_{n}$ and $\left[\mathrm{Ba}_{3} \mathrm{Cu}_{3}(\mathrm{~L})_{6}\left(\mathrm{H}_{2} \mathrm{O}\right)_{11}\right]_{n} \cdot 2 n\left(\mathrm{H}_{2} \mathrm{O}\right)$, where $\mathrm{H}_{2} \mathrm{~L}$ is 1,1-cyclopropanedicarboxylic, 1,1-cyclobutanedicarboxylic and butylmalonic acid, respectively, occurs above $170{ }^{\circ} \mathrm{C}$ and leads to the formation of $\mathrm{BaCuO}_{2} \cdot{ }^{356}$ It is important that the thermolysis conditions have effect on the phase compositions of nanomaterials and the formation of $\mathrm{BaCuO}_{2}$ proceeds under milder conditions from latter CP than those from the first two CPs.

It should be noted isostructural CPs $\left[\mathrm{Ln}(\mathrm{L})(\mathrm{acac})_{2}\left(\mathrm{H}_{2} \mathrm{O}\right)\right]_{n}(\mathrm{Ln}$ $=\mathrm{Eu}, \mathrm{Gd}, \mathrm{Tb}$, Dy, Ho, Er; $\left.\mathrm{L}=\left(\eta^{5}-\mathrm{C}_{5} \mathrm{H}_{4}\right) \mathrm{Mn}(\mathrm{CO})_{3}\right)$, thermolysis of which in air and under Ar atmosphere leads to $\mathrm{LnMnO}_{3}$ phases. ${ }^{357}$ For the Dy and Ho chelates, adiabatic calorimetric data permit to calculate standard thermodynamic functions.

Of interest are 3D-porous CPs $\left[\left(\mathrm{H}_{2} \mathrm{O}\right)_{3}\left(\mu-\mathrm{H}_{2} \mathrm{O}\right)_{2} \mathrm{CuBa}\left(\mu_{3^{-}}\right.\right.$ $\mathrm{L})(\mathrm{L})]_{n}$ and $\left[\left(\mu-\mathrm{H}_{2} \mathrm{O}\right) \mathrm{CuBa}\left(\mu_{3}-\mathrm{L}\right)\left(\mu_{4}-\mathrm{L}\right)\right]_{n}$, where $\mathrm{HL}$ is dimethylmalonic acid. ${ }^{358}$ Complete solid-phase thermolysis of the CPs leads to a mixture of $\mathrm{BaCuO}_{2}, \mathrm{BaCO}_{3}$, and $\mathrm{CuO}$. It is important that a crystalline phase of pure cubic $\mathrm{BaCuO}_{2}$ can be obtained using special conditions. The thermal dehydration of $\mathrm{Ba}-\mathrm{Co}$ and $\mathrm{Ba}-\mathrm{Zn}$ heterometallic $3 \mathrm{D}$ CPs $\left[\mathrm{BaM}\left(\mathrm{H}_{2} \mathrm{O}\right)_{5}(\mathrm{~L})_{2}\right]_{n}$ based on the same ligand occurs in one step. ${ }^{359}$ Crystalline $\mathrm{BaCoO}_{3-x}$ and $\mathrm{BaZnO}_{2}$ are products of solid state thermolysis of the CPs under the following conditions: air flow rate $60 \mathrm{ml} \mathrm{min}{ }^{-1}$, heating rate $10{ }^{\circ} \mathrm{C} \min ^{-1}$ up to $t=800{ }^{\circ} \mathrm{C}(1)$ or
$750{ }^{\circ} \mathrm{C}(2)$, followed by storage for $12 \mathrm{~h}$ at the same temperatures (Fig. 36).

Mesoporous nickel ferrite $\left(\mathrm{NiFe}_{2} \mathrm{O}_{4}\right)$ spheres were prepared by an aerosol spray pyrolysis method using Pluronic F127 as a structure-directing agent, and metal nitrates were used as inorganic precursors for the oxide. ${ }^{\mathbf{3 6 0}}$ Self-assembly of organic and inorganic species followed by metal nitrate decomposition and inorganic polymerization were accomplished in the ethanol aerosol droplets in $\mathrm{N}_{2}$ atmosphere at $400{ }^{\circ} \mathrm{C}$. It is important that the solvent evaporation at $50{ }^{\circ} \mathrm{C}$ in the chamber induced the self-assembly between the structure-directing agent and the metal ions. It should be noted that mesoporous crystalline nickel ferrite spheres were obtained after the spheres were calcined at $300{ }^{\circ} \mathrm{C}$ in air to remove the structure-directing agent and increase crystallinity. Mesoporous $\mathrm{NiFe}_{2} \mathrm{O}_{4}$ spheres of high specific surface area $\left(278 \mathrm{~m}^{2} \mathrm{~g}^{-1}\right)$ with a highly crystalline framework were prepared by adjusting the amount of structuredirecting agent and the calcining condition.

Of interest is the preparation of $\mathrm{Ni}-\mathrm{Fe}$ bimetallic oxide nanotubes with a hollow and porous structure by MOF thermolysis. ${ }^{361}$ It is important that the $\mathrm{Ni} / \mathrm{Fe}$ molar ratios in the binary metal oxide can be regulated. In particular, $\mathrm{Ni}_{0.62} \mathrm{Fe}_{2.38} \mathrm{O}_{4}$ nanotubes with a tube shell of around $10 \mathrm{~nm}$ have a specific surface area of $134.3 \mathrm{~m}^{2} \mathrm{~g}^{-1}$ and are composed of nano-sized primary particles.

Of course, the range of nanostructures produced in the course of thermal transformations of CP is quite wide, and is not limited to the examples discussed here. Huge selection of precursor organometallic structures and coordination polymers with a wide arsenal of synthetic methods for their preparation, ease of many recipes, scalability and acceptable ratio price/quality provide a methodological platform to build on the basis of CP promising new materials including metal nanostructures, nanoalloys, metal chalcogenides, metal carbides, etc.

\section{Concluding remarks and outlook}

As the contents of this review show, the current stage of development of CPs with chelated units has reached its peak in the accumulation of experimental facts and their theoretical
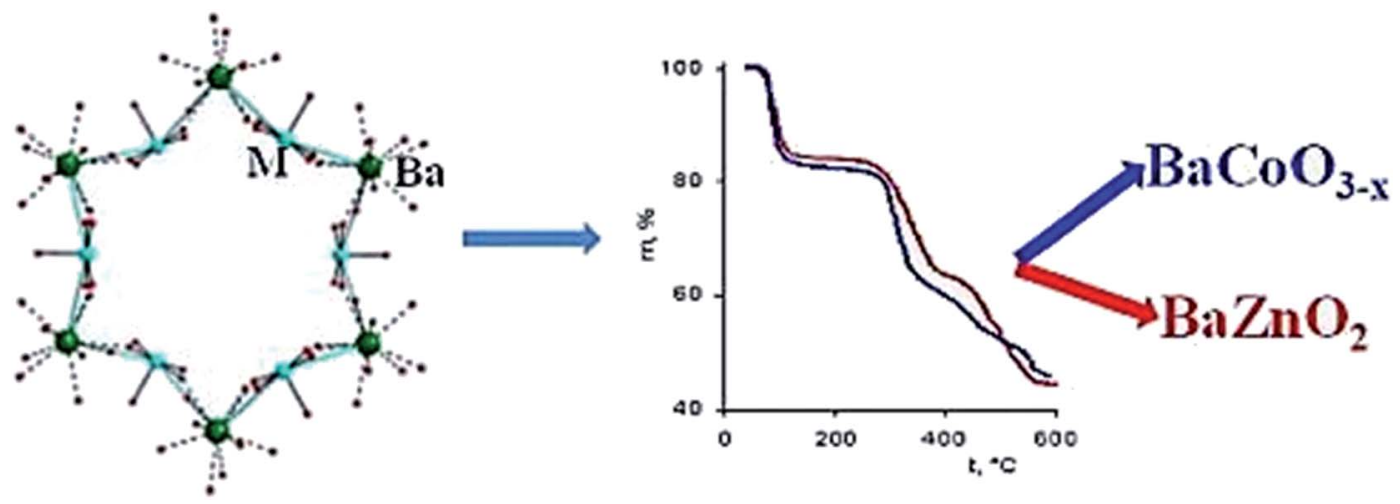

Fig. 36 Preparation of crystalline $\mathrm{BaCoO}_{3-x}$ and $\mathrm{BaZnO}_{2}$ as a result of solid state thermolysis of the CPs. [Reprinted with permission from ref. 359 . Copyright 2015, Elsevier.] 
interpretation and generalization; all major groups of researchers are involved in this area of science.

How we see development of the chemistry of coordination polymers with chelated units?

Firstly, it is the search for more "precision" of the directed synthesis of the polytopic ligands containing chelating sites with controlled flexibility, allowing design of the unstressed metallocycles. In this regard, structural homogeneity of CPs with chelated units and the lack of functional groups capable of forming other coordination centers are important. It is important to develop CPs with chelated units, chelating centers which include non-traditional heteroatoms. The optimization of the synthesis methods of CPs with chelated units will continue by reducing the number of synthetic steps (for example, by combining the synthesis of chelating polytopic ligands and CPS on their basis, etc.).

It should be noted that the CPs with chelated units make important contribution to developing the chemistry of polymeric metal chelates. Among the most important tasks in this direction are the continued development and optimization of low cost, high-yielding synthetic routes toward final CP with minimal impurities or contaminations; the continued introduction of new "green" chemistries and solvent-free or aqueous solution of preparation processes (in the absence of organic solvents) into synthetic methodologies of $\mathrm{CP}$, which would minimize the cost and environmental impact; control over sitespecific functionality (for example, core-shell-periphery); design of shape/morphology changing CP on application of an external stimulus; further investigation into CP for targeted biological applications.

The promising way will be further development of nanomaterials preparation by thermolysis of CPs. This is an intensely developed field of chemistry and materials science, which possesses its objects and investigation methods. Information in this field is accumulated continuously collecting the facts that seemed strange earlier and completing logical structuring of new ways of production of nanomaterials with high marketing potential. Nevertheless, till now, as a rule, it is impossible to recognize correlations between content, structural features, and properties of the nanomaterials prepared by this method, which in many respects restrains scientifically grounded approach to structuring of these materials and prediction of their promising properties.

\section{Conflicts of interest}

No potential conflict of interest was reported by the authors.

\section{References}

1 K. Naka, Metal Organic Framework (MOF), in Encyclopedia of Polymeric Nanomaterials, ed. S. Kobayashi and $\mathrm{K}$. Müllen, Springer, 2015.

2 Metal-Organic Frameworks: Materials Modeling towards Engineering Applications, ed. J. Jiang, CRC, 2015.
3 R. Zhai, F. Jiao, H. Lin, F. Hao, J. Li, H. Yan, N. Li, H. Wang, Z. Jin, Y. Zhang and Õ. Qian, Chin. J. Chromatogr., 2014, 32, 107-116.

4 Metal-Organic Framework Materials, ed. L. R. MacGillivray and C. M. Lukehart, Wiley, 2014.

5 H.-C. Zhou, J. R. Long and O. M. Yaghi, Chem. Rev., 2012, 112, 673-674.

6 W. Lu, Z. Wei, Z.-Y. Gu, T.-F. Liu, J. Park, J. Park, J. Tian, M. Zhang, Q. Zhang, T. Gentle III, M. Bosch and H.-C. Zhou, Chem. Soc. Rev., 2014, 43, 5561-5593.

7 O. L. Ortiz and L. D. Ramírez, Coordination Polymers and Metal Organic Frameworks: Properties, Types, and Applications, Nova Science Publishers, 2012.

8 M. O'Keeffe and O. M. Yaghi, Chem. Rev., 2012, 112, 675702.

9 S.-L. Wu, J.-P. Zou, M.-H. Chen, H.-B. Yang, M.-J. Li, X.-B. Luo, F. Luo, M.-F. Wu and G.-C. Guo, Polyhedron, 2012, 48, 58-67.

10 R. Haldar, N. Sikdar and T. K. Maji, Mater. Today, 2015, 18, 97-116.

11 K. E. Knope, D. T. Lill, C. E. Rowland, P. M. Cantos, A. Bettencourt-Dias and C. L. Cahill, Inorg. Chem., 2012, 51, 201-206.

12 W. Q. Kan, J. F. Ma, Y. Y. Liu and J. Yang, CrystEngComm, 2012, 14, 2316-2326.

13 J.-R. Li, J. Sculley and H.-C. Zhou, Chem. Rev., 2012, 112, 869-932.

14 H. Wu, Q. Gong, D. H. Olson and J. Li, Chem. Rev., 2012, 112, 836-868.

15 K. Sumida, D. L. Rogow, J. A. Mason, T. M. McDonald, E. D. Bloch, Z. R. Herm, T.-H. Bae and J. R. Long, Chem. Rev., 2012, 112, 724-781.

16 M. P. Suh, H. J. Park, T. K. Prasad and D.-W. Lim, Chem. Rev., 2012, 112, 782-835.

17 Y. He, W. Zhou, G. Qian and B. Chen, Chem. Soc. Rev., 2014, 43, 5657-5678.

18 S. Qiu, M. Xue and G. Zhu, Chem. Soc. Rev., 2014, 43, 61166140.

19 H.-J. Son, S. Jin, S. Patwardhan, S. J. Wezenberg, N. C. Jeong, M. So, C. E. Wilmer, A. A. Sarjeant, G. C. Schatz, R. Q. Snurr, O. K. Farha, G. P. Wiederrecht and J. T. Hupp, J. Am. Chem. Soc., 2013, 135, 862-869.

20 S. Jin, H.-J. Son, O. K. Farha, G. P. Wiederrecht and J. T. Hupp, J. Am. Chem. Soc., 2013, 135, 955-958.

21 P. Horcajada, R. Gref, T. Baati, P. K. Allan, G. Maurin, P. Couvreur, G. Férey, R. E. Morris and C. Serre, Chem. Rev., 2012, 112, 1232-1268.

22 W. Morris, B. Volosskiy, S. Demir, F. Gándara, P. L. McGrier, H. Furukawa, D. Cascio, J. F. Stoddart and O. M. Yaghi, Inorg. Chem., 2012, 51, 6443-6445.

23 J. Liu, P. K. Thallapally, B. P. McGrail, D. R. Brown and J. Liu, Chem. Soc. Rev., 2012, 41, 2308-2322.

24 C. He, D. Liu and W. Lin, Chem. Rev., 2015, 115, 1107911108.

25 K. Lu, C. He and W. Lin, J. Am. Chem. Soc., 2015, 137, 76007603. 
26 Z. Hu, B. J. Deibert and J. Li, Chem. Soc. Rev., 2014, 43, 58155840.

27 L. E. Kreno, K. Leong, O. K. Farha, M. Allendorf, R. P. Van Duyne and J. T. Hupp, Chem. Rev., 2012, 112, 11051125.

28 Y. Cui, Y. Yue, G. Qian and B. Chen, Chem. Rev., 2012, 112, 1126-1162.

29 J. Heine and K. Muller-Buschbaum, Chem. Soc. Rev., 2013, 42, 9232-9242.

30 M. Yoon, R. Srirambalaji and K. Kim, Chem. Rev., 2012, 112, 1196-1231.

31 J. Liu, L. Chen, H. Cui, J. Zhang, L. Zhang and C.-Y. Su, Chem. Soc. Rev., 2014, 43, 6011-6061.

32 P. Phuengphai, S. Youngme, P. Gamez and J. Reedijk, Dalton Trans., 2010, 39, 7936-7942.

33 T. Zhang and W. Lin, Chem. Soc. Rev., 2014, 43, 5982-5993.

34 A. H. Chughtai, N. Ahmad, H. A. Younus, A. Laypkov and F. Verpoort, Chem. Soc. Rev., 2015, 44, 6804-6849.

35 J. Canivet, S. Aguado, Y. Schuurman and D. Farrusseng, J. Am. Chem. Soc., 2013, 135, 4195-4198.

36 A. N. Mlinar, B. K. Keitz, D. Gygi, E. D. Bloch, J. R. Long and A. T. Bell, ACS Catal., 2014, 4, 717-721.

37 Y. Zhang, Y. Zhou, Y. Zhao and C. Liu, Catal. Today, 2016, 263, 61-68.

38 A. Bhunia, S. Dey, J. M. Moreno, U. Diaz, P. Concepcion, K. Van Hecke, C. Janiak and P. Van Der Voort, Chem. Commun., 2016, 52, 1401-1404.

39 S. Ou and C.-D. Wu, Inorg. Chem. Front., 2014, 1, 721-734. 40 P. Ramaswamy, N. E. Wong and G. K. H. Shimizu, Chem. Soc. Rev., 2014, 43, 5913-5932.

41 C.-Y. Sun, X.-L. Wang, X. Zhang, C. Qin, P. Li, Z.-M. Su, D.-X. Zhu, G.-G. Shan, K.-Z. Shao, H. Wu and J. Li, Nat. Commun., 2013, 4, 2717.

42 Y. Cui, F. Zhu, B. Chen and G. Qian, Chem. Commun., 2015, 51, 7420-7431.

43 J. Pan, C.-P. Liu, F.-L. Jiang, M.-Y. Wu, L. Chen, J.-J. Qian, K.-Z. Su, X.-Y. Wan and M.-C. Hong, CrystEngComm, 2015, 17, 1541-1548.

44 X.-P. Wang, T.-P. Hu and D. Sun, CrystEngComm, 2015, 17, 3393-3417.

45 A. D. Pomogailo and G. I. Dzhardimalieva, Nanostructured Materials Preparation via Condensation Ways, Springer, 2014.

46 A. D. Pomogailo and G. I. Dzhardimalieva, Metallopolymeric Hybrid Nanocomposities, Nauka, 2015.

47 B. I. Kharisov, O. V. Kharissova and U. O. Méndez, J. Coord. Chem., 2013, 66, 3791-3828.

48 O. Carp, Materials Obtained by Solid-State Thermal Decomposition of Coordination Compounds and MetalOrganic Coordination Polymers, in Reactions and Mechanisms in Thermal Analysis of Advanced Materials, ed. A. Tiwari and B. Raj, Scrivener Publishing LLC, 2015.

49 Y. Song, X. Li, L. Sun and L. Wang, RSC Adv., 2015, 5, 72677279.

50 J.-K. Sun and Q. Xu, Energy Environ. Sci., 2014, 7, 2071-2100.
51 B. I. Kharisov, H. V. Rasika Dias, O. V. Kharissova, V. M. Jiménez-Pérez, B. O. Pérez and B. M. Flores, $R S C$ Adv., 2012, 2, 9325-9358.

52 M. Y. Masoomi and A. Morsali, Coord. Chem. Rev., 2012, 256, 2921-2943.

53 A. D. Pomogailo and I. E. Uflyand, Adv. Polym. Sci., 1990, 97, 61-105.

54 A. D. Pomogailo and I. E. Uflyand, Makromolekulayrnye Metallokhelaty Macromolecular Metal Chelates, Khimiya, 1991.

55 G. Kumar and R. Gupta, Chem. Soc. Rev., 2013, 42, 94039453.

56 D. Feng, Z.-Y. Gu, J.-R. Li, H.-L. Jiang, Z. Wei and H.-C. Zhou, Angew. Chem., Int. Ed., 2012, 51, 10307-10310.

57 Y. Chen, T. Hoang and S. Ma, Inorg. Chem., 2012, 51, 1260012602.

58 X.-S. Wang, M. Chrzanowski, C. Kim, W.-Y. Gao, L. Wojtas, Y.-S. Chen, X. P. Zhang and S. Ma, Chem. Commun., 2012, 48, 7173-7175.

59 X.-S. Wang, M. Chrzanowski, W.-Y. Gao, L. Wojtas, Y.-S. Chen, M. J. Zaworotko and S. Ma, Chem. Sci., 2012, 3, 2823-2827.

60 X.-S. Wang, M. Chrzanowski, L. Wojtas, Y.-S. Chen and S. Ma, Chem.-Eur. J., 2013, 19, 3297-3301.

61 L. Meng, Q. Cheng, C. Kim, W.-Y. Gao, L. Wojtas, Y.-S. Cheng, M. J. Zaworotko, X. P. Zhang and S. Ma, Angew. Chem., Int. Ed., 2012, 51, 10082-10085.

62 X.-L. Yang, M.-H. Xie, C. Zou, Y. He, B. Chen, M. O'Keeffe and C.-D. Wu, J. Am. Chem. Soc., 2012, 134, 10638-10645.

63 A. Fateeva, P. A. Chater, C. P. Ireland, A. A. Tahir, Y. Z. Khimyak, P. V. Wiper, J. R. Darwent and M. J. Rosseinsky, Angew. Chem., Int. Ed., 2012, 51, 74407444.

64 V. S. P. K. Neti, A. J. Metta-Magaña and L. Echegoyen, J. Coord. Chem., 2013, 66, 3193-3198.

65 D. Feng, H.-L. Jiang, Y.-P. Chen, Z.-Y. Gu, Z. Wei and H.-C. Zhou, Inorg. Chem., 2013, 52, 12661-12667.

66 J. A. Johnson, X. Zhang, T. C. Reeson, Y. S. Chen and J. Zhang, J. Am. Chem. Soc., 2014, 136, 15881-15884.

67 B. J. Burnett and W. Choe, CrystEngComm, 2012, 14, 61296131.

68 J. M. Falkowski, T. Sawano, T. Zhang, G. Tsun, Y. Chen, J. V. Lockard and W. Lin, J. Am. Chem. Soc., 2014, 136, 5213-5216.

69 Z. Zhang, L. Wojtas, M. Eddaoudi and M. J. Zaworotko, J. Am. Chem. Soc., 2013, 135, 5982-5985.

70 Z. Zhang and M. J. Zaworotko, Chem. Soc. Rev., 2014, 43, 5444-5455.

71 Z. Zhang, L. Zhang, L. Wojtas, P. Nugent, M. Eddaoudi and M. J. Zaworotko, J. Am. Chem. Soc., 2012, 134, 924-927.

72 Z. Zhang, L. Zhang, L. Wojtas, M. Eddaoudi and M. J. Zaworotko, J. Am. Chem. Soc., 2012, 134, 928-933.

73 A. Fidalgo-Marijuan, G. Barandika, B. Bazán, M.-K. Urtiaga and M. I. Arriortua, CrystEngComm, 2013, 15, 4181-4188.

74 L. Xu, J. Wang, Y. Xu, Z. Zhang, P. Lu, M. Fang, S. Li, P. Sun and H.-K. Liu, CrystEngComm, 2014, 16, 8656-8659. 
75 B. J. Burnett, P. M. Barron, C. Hu and W. Choe, Eur. J. Inorg. Chem., 2012, 2012, 4885-4897.

76 K. Wang, D. Feng, T.-F. Liu, J. Su, S. Yuan, Y.-P. Chen, M. Bosch, X. Zou and H.-C. Zhou, J. Am. Chem. Soc., 2014, 136, 13983-13986.

77 H. Deng, S. Grunder, K. E. Cordova, C. Valente, H. Furukawa, M. Hmadeh, F. Gandara, A. C. Whalley, Z. Liu, S. Asahina, H. Kazumori, M. O'Keeffe, O. Terasaki, J. F. Stoddart and O. M. Yaghi, Science, 2012, 336, 10181023.

78 J. An, O. K. Farha, J. T. Hupp, E. Pohl, J. I. Yeh and N. L. Rosi, Nat. Commun., 2012, 3, 604.

79 D. Feng, W.-C. Chung, Z. Wei, Z.-Y. Gu, H.-L. Jiang, Y.-P. Chen, D. J. Darensbourg and H.-C. Zhou, J. Am. Chem. Soc., 2013, 135, 17105-17110.

80 W.-Y. Gao, M. Chrzanowski and S. Ma, Chem. Soc. Rev., 2014, 43, 5841-5866.

81 C. Zou, T. Zhang, M.-H. Xie, L. Yan, G.-Q. Kong, X.-L. Yang, A. Ma and C.-D. Wu, Inorg. Chem., 2013, 52, 3620-3626.

82 C. Zou, M.-H. Xie, G.-Q. Kong and C.-D. Wu, CrystEngComm, 2012, 14, 4850-4856.

83 M.-H. Xie, X.-L. Yang, Y. He, J. Zhang, B. Chen and C.-D. Wu, Chem.-Eur. J., 2013, 19, 14316-14321.

84 S. Matsunaga, N. Endo and W. Mori, Eur. J. Inorg. Chem., 2012, 2012, 4885-4897.

85 K. Y. Lee, Y. S. Lee, S. Kim, H. M. Ha, S.-E. Bae, S. Huh, H. G. Jang and S. J. Lee, CrystEngComm, 2013, 15, 93609363.

86 Z. Guo, D. Yan, H. Wang, D. Tesfagaber, X. Li, Y. Chen, W. Huang and B. Chen, Inorg. Chem., 2015, 54, 200-204.

87 Z. Zhang, J. Li, Y. Yao and S. Sun, Cryst. Growth Des., 2015, 15, 5028-5033.

88 C. Zhu, G. Yuan, X. Chen, Z. Yang and Y. Cui, J. Am. Chem. Soc., 2012, 134, 8058-8061.

89 Z.-Y. Gu, J. Park, A. Raiff, Z. Wei and H.-C. Zhou, ChemCatChem, 2014, 6, 67-75.

90 A. Bhunia, M. A. Gotthardt, M. Yadav, M. T. Gamer, A. Eichöfer, W. Kleist and P. W. Roesky, Chem.-Eur. J., 2013, 19, 1986-1995.

91 M. C. Das, Q. Guo, Y. He, J. Kim, C.-G. Zhao, K. Hong, S. Xiang, Z. Zhang, K. M. Thomas, R. Krishna and B. Chen, J. Am. Chem. Soc., 2012, 134, 8703-8710.

92 A. Ali, G. Hundal and R. Gupta, Cryst. Growth Des., 2012, 12, 1308-1319.

93 G. Kumar, H. Aggarwal and R. Gupta, Cryst. Growth Des., 2013, 13, 74-90.

94 S. Srivastava, A. Ali, A. Tyagi and R. Gupta, Eur. J. Inorg. Chem., 2014, 2014, 2113-2123.

95 S. Srivastava, M. S. Dagur and R. Gupta, Eur. J. Inorg. Chem., 2014, 2014, 4966-4974.

96 G. Kumar and R. Gupta, Inorg. Chem. Commun., 2012, 23, 103-108.

97 D. Bansal, G. Hundal and R. Gupta, Eur. J. Inorg. Chem., 2015, 2015, 1022-1032.

98 G. Kumar and R. Gupta, Inorg. Chem., 2012, 51, 54975499.
99 G. Kumar and R. Gupta, Inorg. Chem., 2013, 52, 1077310787.

100 L. K. Das, A. M. Kirillov and A. Ghosh, CrystEngComm, 2014, 16, 3029-3039.

101 Y.-L. Hou, S.-X. Li, R. W.-Y. Sun, X.-Y. Liu, S. W. Ng and D. Li, Dalton Trans., 2015, 44, 17360-17365.

102 Y.-L. Hou, R. W.-Y. Sun, X.-P. Zhou, J.-H. Wang and D. Li, Chem. Commun., 2014, 50, 2295-2297.

103 Q.-L. Zhang, G.-W. Feng, Y.-Q. Zhang and B.-X. Zhu, RSC Adv., 2014, 4, 11384-11392.

104 A. D. Burrows, M. F. Mahon, C. L. Renouf, C. Richardson, A. J. Warren and J. E. Warren, Dalton Trans., 2012, 41, 4153-4163.

105 C. Mohapatra and V. Chandrasekhar, Cryst. Growth Des., 2014, 14, 406-409.

106 S. Srivastava, H. Aggarwal and R. Gupta, Cryst. Growth Des., 2015, 15, 4110-4122.

107 A. Kobayashi, A. Sugiyama, T. Ohba, Y. Suzuki, H.-C. Chang and M. Kato, Chem. Lett., 2014, 43, 1070-1072.

108 A. Kobayashi, Y. Suzuki, T. Ohba, T. Ogawa, T. Matsumoto, S.-i. Noro, H.-C. Chang and M. Kato, Inorg. Chem., 2015, 54, 2522-2535.

109 H.-L. Wang, S.-B. Wu, X.-M. Shi, X.-Z. Li, P.-P. Hao and L.-N. Zhu, J. Inorg. Organomet. Polym., 2015, 25, 730-738.

110 C. Zou and C.-D. Wu, Dalton Trans., 2012, 41, 3879-3888.

111 L. Wang, J. Morales, T. Wu, X. Zhao, W. P. Beyermann, X. Bu and P. Feng, Chem. Commun., 2012, 48, 7498-7500.

112 D.-X. Xue, A. J. Cairns, Y. Belmabkhout, Ł. Wojtas, Y. Liu, M. H. Alkordi and M. Eddaoudi, J. Am. Chem. Soc., 2013, 135, 7660-7667.

113 J. Eubank, F. Nouar, R. Luebke, A. Cairns, L. Wojtas, M. Alkordi, T. Bousquet, M. Hight, J. Eckert, J. Embs, P. Georgiev and M. Eddaoudi, Angew. Chem., Int. Ed., 2012, 51, 10099-10103.

114 R. Luebke, J. F. Eubank, A. J. Cairns, Y. Belmabkhout, L. Wojtas and M. Eddaoudi, Chem. Commun., 2012, 48, 1455-1457.

115 R. Luebke, Ł. J. Weseliński, Y. Belmabkhout, Z. Chen, Ł. Wojtas and M. Eddaoudi, Cryst. Growth Des., 2014, 14, 414-418.

116 V. Guillerm, Ł. J. Weselinski, Y. Belmabkhout, A. J. Cairns, V. D'Elia, Ł. Wojtas, K. Adil and M. Eddaoudi, Nat. Chem., 2014, 6, 673-680.

117 U. Stoeck, S. Krause, V. Bon, I. Senkovska and S. Kaskel, Chem. Commun., 2012, 48, 10841-10843.

118 V. Guillerm, D. Kim, J. F. Eubank, R. Luebke, X. Liu, K. Adil, M. S. Lah and M. Eddaoudi, Chem. Soc. Rev., 2014, 43, 61416172.

119 Z. Chen, K. Adil, Ł. J. Weseliński, Y. Belmabkhout and M. Eddaoudi, J. Mater. Chem. A, 2015, 3, 6276-6281.

120 N. Stock and S. Biswas, Chem. Rev., 2012, 112, 933-969.

121 Y.-R. Lee, J. Kim and W.-S. Ahn, Korean J. Chem. Eng., 2013, 30, 1667-1680.

122 Y. Sun and H.-C. Zhou, Sci. Technol. Adv. Mater., 2015, 16, 054202. 
123 C. Dey, T. Kundu, B. P. Biswal, A. Mallick and R. Banerjee, Acta Crystallogr., Sect. B: Struct. Sci., Cryst. Eng. Mater., 2014, 70, 3-10.

124 B. Seoane, S. Castellanos, A. Dikhtiarenko, F. Kapteijn and J. Gascon, Coord. Chem. Rev., 2016, 307, 147-187.

125 M. Mishra, K. Tiwari, A. K. Singh and V. P. Singh, Inorg. Chim. Acta, 2015, 425, 36-45.

126 G. Yuan, G. Hu, W. Shan, S. Jin, Q. Gu and J. Chen, Dalton Trans., 2015, 44, 17774-17783.

127 F. Y. Wardana, S.-W. Ng and A. C. Wibowo, Cryst. Growth Des., 2015, 15, 5930-5938.

128 H. L. Jiang, D. Feng, K. Wang, Z. Y. Gu, Z. Wei, Y. P. Chen and H. C. Zhou, J. Am. Chem. Soc., 2013, 135, 13934-13938.

129 B. Machura, J. Palion, A. Šwitlicka, J. Mroziński, B. Kalińska and R. Kruszyński, Polyhedron, 2013, 49, 216-222.

130 Z. Abulizi, L. Wang, S. Touhuti, Y. Zhang, Y. Wang and Y. Huaxue, Chin. J. Appl. Chem., 2012, 29, 938-941.

131 S. Banerjee, P. Rajakannu, R. J. Butcher and R. Murugavel, CrystEngComm, 2014, 16, 8429-8441.

132 G.-P. Yang, Y.-Y. Wang, P. Liu, A.-Y. Fu, Y.-N. Zhang, J.-C. Jin and Q.-Z. Shi, Cryst. Growth Des., 2010, 10, 1443-1450.

133 X.-L. Wang, F.-F. Sui, H.-Y. Lin, J.-W. Zhang and G.-C. Liu, Cryst. Growth Des., 2014, 14, 3438-3452.

134 X. Li, M. Fang, P. Cui and B. Zhao, Z. Anorg. Allg. Chem., 2013, 639, 626-632.

135 Y. Ren, H. Jiang, B. Cai and J. Lu, Inorg. Chem. Commun., 2012, 24, 114-117.

136 W. Guo, C.-H. Jiang and Y.-M. Guo, Inorg. Chim. Acta, 2013, 405, 128-133.

137 W. Guo, A. Escuer, M. Tang, C.-H. Jiang and M. Du, Inorg. Chim. Acta, 2013, 403, 142-148.

138 X. Wang and Y.-M. Guo, Inorg. Chem. Commun., 2015, 60, 115-118.

139 X. Wang, W. Guo and Y.-M. Guo, J. Mol. Struct., 2015, 1096, 136-141.

140 N. J. Hurley, J. M. Rawson and M. Pilkington, Dalton Trans., 2015, 44, 1866-1874.

141 Y. Song, M.-L. Feng, Z.-F. Wu and X.-Y. Huang, CrystEngComm, 2015, 17, 1348-1357.

142 M. Sánchez-Sánchez, N. Getachew, K. Díaz, M. Díaz-García, Y. Chebude and I. Díaz, Green Chem., 2015, 17, 1500-1509.

143 D. Cai, H. Guo, L. Wen and C. Liu, CrystEngComm, 2013, 15, 6702-6708.

144 H.-Q. Xu, K. Wang, M. Ding, D. Feng, H.-L. Jiang and H.-C. Zhou, J. Am. Chem. Soc., 2016, 138, 5316-5319.

145 B. Tao, X. Jiang, H. Xia and H. Cheng, J. Mol. Struct., 2012, 1011, 15-18.

146 F. A. A. Paz, J. Klinowski, S. M. F. Vilela, J. P. C. Tome, J. A. S. Cavaleiro and J. Rocha, Chem. Soc. Rev., 2012, 41, 1088-1110.

147 S. T. Zheng, T. Wu, C. T. Chou, A. Fuhr, P. Y. Feng and X. H. Bu, J. Am. Chem. Soc., 2012, 134, 4517-4520.

148 Y. Liu, M. J. W. Tan, F. Wei, Y. Tian, T. Wu, C. Kloc, F. Huo, Q. Yan, H. H. Hng, J. Ma and Q. Zhang, CrystEngComm, 2012, 14, 75-78.

149 Y. Liu, L.-M. Yu, S. C. J. Loo, R. G. Blair and Q. Zhang, J. Solid State Chem., 2012, 191, 283-286.
150 D.-C. Zhong, H.-B. Guo, J.-H. Deng, Q. Chen and X.-Z. Luo, CrystEngComm, 2015, 17, 3519-3525.

151 Z. Wei, X. Mu and Y. Chen, Synth. React. Inorg., Met.-Org., Nano-Met. Chem., 2016, 46, 147-150.

152 Y. Xu, Y.-X. Che and J.-M. Zheng, Z. Anorg. Allg. Chem., 2012, 638, 698-701.

153 J. Zhao, P. Su, Y. Zhao, M. Li, Y. Yang, Q. Yang and C. Li, J. Mater. Chem., 2012, 22, 8470-8475.

154 A. J. Calahorro, D. Fairen-Jiménez, A. Salinas-Castillo, M. E. López-Viseras and A. Rodríguez-Diéguez, Polyhedron, 2013, 52, 315-320.

155 Z. Shen, S. He, P. Yao, X. Lao, B. Yang, Y. Dai, X. Sun and T. Chen, RSC Adv., 2014, 4, 12844-12848.

156 L. Fan, H. Chen, D. Xiao and E. Z. Wang, Z. Anorg. Allg. Chem., 2013, 639, 558-561.

157 B.-Y. Zhu, X.-L. Zhang, F. Guo and X.-H. Liu, Inorg. Chim. Acta, 2012, 391, 58-65.

158 Z.-F. Li, X.-B. Luo, Y.-C. Gao, H.-J. Lu and G. Li, Inorg. Chim. Acta, 2012, 384, 352-360.

159 Y. Lou, J. Wang, Y. Tao, J. Chen, A. Mishima and M. Ohba, Dalton Trans., 2014, 43, 8508-8514.

160 A.-T. Pu, J. Yang, W.-Q. Kan, Y. Yang and J.-F. Ma, Polyhedron, 2013, 50, 556-570.

161 X. Zhang, L. Fan, Z. Sun, W. Zhang, D. Li, P. Wei, B. Li and J. Dou, J. Coord. Chem., 2012, 65, 3205-3215.

162 X.-H. Chang, L.-F. Ma, G. Hui and L.-Y. Wang, Cryst. Growth Des., 2012, 12, 3638-3646.

163 M. Kariem, M. Yawer and H. N. Sheikh, J. Solid State Chem., 2015, 231, 239-247.

164 H.-W. Kuai, X.-C. Cheng and X.-H. Zhu, Polyhedron, 2013, 50, 390-397.

165 H.-W. Kuai, C. Hou and W.-Y. Sun, Polyhedron, 2013, 52, 1268-1275.

166 L. Yang, L. Liu, C. Lian, M. Liu, Z. Xu, L.-C. Wang, X. Guo and Y. Long, Dyes Pigm., 2015, 122, 246-255.

167 R. F. D'Vries, M. Iglesias, N. Snejko, E. Gutiérrez-Puebla and M. Angeles Monge, Inorg. Chem., 2012, 51, 11349-11355.

168 X. Wang, Z. Guo, G. Liu, S. Yang, Y. Qu, H. Lin and J. Zhang, J. Coord. Chem., 2012, 65, 2634-2644.

169 L. Wu, Y. Sun, X. Liu, D. Gao, Y. Xu and G. Zhang, Chem. Res. Chin. Univ., 2012, 28, 775-779.

170 P. K. Yadav, N. Kumari, P. Pachfule, R. Banerjee and L. Mishra, Cryst. Growth Des., 2012, 12, 5311-5319.

171 Z. Zhang, J. Li, R. Liu, W. Zhou and F. Zhang, J. Coord. Chem., 2013, 66, 926-936.

172 M. Fang, P.-F. Shi, B. Zhao, D.-X. Jiang, P. Cheng and W. Shi, Dalton Trans., 2012, 41, 6820-6826.

173 A. L. Ramirez, K. E. Knope, T. T. Kelley, N. E. Greig, J. D. Einkauf and D. T. de Lill, Inorg. Chim. Acta, 2012, 392, 46-51.

174 Y.-Y. Liu, B. Liu, J. Yang and J.-F. Ma, Polyhedron, 2013, 56, 96-101.

175 R. Decadt, K. Van Hecke, D. Depla, K. Leus, D. Weinberger, I. Van Driessche, P. Van Der Voort and R. Van Deun, Inorg. Chem., 2012, 51, 11623-11634.

176 K. Wang, Z. Geng, M. Zheng, L. Ma, X. Ma and Z. Wang, Cryst. Growth Des., 2012, 12, 5606-5614. 
177 F.-J. Meng, H.-Q. Jia, N.-H. Hu and J.-W. Xu, Inorg. Chem. Commun., 2012, 21, 186-190.

178 L. Yang, S. Song, H. Zhang, W. Zhang, L. Wu, Z. Bu and T. Ren, Synth. Met., 2012, 162, 261-267.

179 S. Song, C. Shao, H. Zhang, W. Zhang, L. Yang, T. Ren and Z. Bu, Synth. React. Inorg., Met.-Org., Nano-Met. Chem., 2013, 43, 169-174.

180 X.-Q. Zhao and Y.-C. Li, J. Coord. Chem., 2013, 66, 937-945. 181 R. K. Vakiti, B. D. Garabato, N. P. Schieber, M. J. Rucks, Y. Cao, C. Webb, J. B. Maddox, A. Celestian, W.-P. Pan and B. Yan, Cryst. Growth Des., 2012, 12, 3937-3943.

182 K. Yang, J.-H. Luo and Z.-H. Liu, Inorg. Chim. Acta, 2012, 391, 206-209.

183 C. Cruz, E. Spodine, A. Vega, D. Venegas-Yazigi and V. Paredes-García, Cryst. Growth Des., 2016, 16, 2173.

184 X. Cao, Y. Liu, L. Wang and G. Li, Inorg. Chim. Acta, 2012, 392, 16-24.

185 B. Roy, S. Mukherjee and P. S. Mukherjee, CrystEngComm, 2013, 15, 9596-9602.

186 L. Yang, L. Zeng, W. Gu, J. Tian, S. Liao, M. Zhang, X. Wei, L. Xin and X. Liu, Inorg. Chem. Commun., 2013, 29, 76-81.

187 K. Cheng and X. L. Zhang, Russ. J. Coord. Chem., 2013, 39, 458-462.

188 K. Suh, M. P. Yutkin, D. N. Dybtsev, V. P. Fedin and K. Kim, Chem. Commun., 2012, 48, 513-515.

189 T. Kawamoto, N. Suzuki, T. Ono, D. Gong and T. Konno, Chem. Commun., 2013, 49, 668-670.

190 A. J. McConnell, C. S. Wood, P. P. Neelakandan and J. R. Nitschke, Chem. Rev., 2015, 115, 7729-7793.

191 Z. L. Luo and S. G. Zhang, Chem. Soc. Rev., 2012, 41, 47364754.

192 F. Vidal, R. R. Gowda and E. Y. X. Chen, J. Am. Chem. Soc., 2015, 137, 9469-9480.

193 S. C. Xu, Y. Magoon, R. R. Reinig, B. M. Schmidt, A. Ellern and A. D. Sadow, Organometallics, 2015, 34, 3508-3519.

194 H. Tan, Y. Li, W. Chen, A. Yan, D. Liu and E. Wang, Cryst. Growth Des., 2012, 12, 1111-1117.

195 L. L. Yan, C. H. Tan, G. L. Zhang, L. P. Zhou, J. C. Bunzli and Q. F. Sun, J. Am. Chem. Soc., 2015, 137, 8550-8555.

196 B. M. Trost and M. Rao, Angew. Chem., Int. Ed., 2015, 54, 5026-5043.

197 W. R. Xu, G. J. Xia, H. F. Chow, X. P. Cao and D. Kuck, Chem.-Eur. J., 2015, 21, 12011-12017.

198 J. P. Byrne, J. A. Kitchen, J. E. O'Brien, R. D. Peacock and T. Gunnlaugsson, Inorg. Chem., 2015, 54, 1426-1439.

199 J. He, G. Zhang, D. Xiao, H. Chen, S. Yan, X. Wang, J. Yanga and E. Wang, CrystEngComm, 2012, 14, 3609-3614.

200 Y. Gong, T. Wu, J. Lin and B. Wang, CrystEngComm, 2012, 14, 5649-5656.

201 S.-L. Cai, S.-R. Zheng, Z.-Z. Wen, J. Fan and W.-G. Zhang, Cryst. Growth Des., 2012, 12, 2355-2361.

202 Z. Chen, S. Qin, D. Liu, Y. Shen and F. Liang, Cryst. Growth Des., 2013, 13, 3389-3395.

203 B. Joarder, A. K. Chaudhari and S. K. Ghosh, Inorg. Chem., 2012, 51, 4644-4649.

204 L. Song, C. Jiang, C. Ling, Y.-R. Yao, Q.-H. Wang and D. Chen, J. Inorg. Organomet. Polym., 2016, 26, 320-325.
205 W. Chen, S.-Q. Su, S.-Q. Song, X.-Z. Song, H.-J. Zhang, G. Xuexiao and H. Xuebao, Chem. J. Chin. Univ., 2012, 33, 215-218.

206 Z. Xu, H. Li, A. Li, W. Menga, H. Hou and Y. Fan, Inorg. Chem. Commun., 2013, 36, 126-129.

207 A. A. Sinelshchikova, S. E. Nefedov, Y. Y. Enakieva, Y. G. Gorbunova, A. Y. Tsivadze, K. M. Kadish, P. Chen, A. Bessmertnykh-Lemeune, C. Stern and R. Guilard, Inorg. Chem., 2013, 52, 999-1006.

208 J. A. Johnson, Q. Lin, L.-C. Wu, N. Obaidi, Z. L. Olson, T. C. Reeson, Y.-S. Chen and J. Zhang, Chem. Commun., 2013, 49, 2828-2830.

209 N. C. Smythe, D. P. Butler, C. E. Moore, W. R. McGowan, A. L. Rheingold and L. G. Beauvais, Dalton Trans., 2012, 41, 7855-7858.

210 J. Park, D. Feng, S. Yuan and H.-C. Zhou, Angew. Chem., Int. Ed., 2015, 54, 430-435.

211 Z. Guo and B. Chen, Dalton Trans., 2015, 44, 1457414583.

212 A. Fateeva, J. Clarisse, G. Pilet, J.-M. Grenèche, F. Nouar, B. K. Abeykoon, F. Guegan, C. Goutaudier, D. Luneau, J. E. Warren, M. J. Rosseinsky and T. Devic, Cryst. Growth Des., 2015, 15, 1819-1826.

213 W.-Y. Gao, Z. Zhang, L. Cash, L. Wojtas, Y.-S. Chen and S. Ma, CrystEngComm, 2013, 15, 9320-9323.

214 W. Zhang, P. Jiang, Y. Wang, J. Zhang and P. Zhang, Inorg. Chem. Commun., 2015, 61, 100-104.

215 Q. Zha, C. Ding, X. Rui and Y. Xie, Cryst. Growth Des., 2013, 13, 4583-4590.

216 X. Rui, Q.-Z. Zha, T.-T. Wei and Y.-S. Xie, Inorg. Chem. Commun., 2014, 48, 111-113.

217 C.-W. Kung, T.-H. Chang, L.-Y. Chou, J. T. Hupp, O. K. Farha and K.-C. Ho, Electrochem. Commun., 2015, $58,51-56$.

218 D. Feng, K. Wang, Z. Wei, Y.-P. Chen, C. M. Simon, R. K. Arvapally, R. L. Martin, M. Bosch, T.-F. Liu, S. Fordham, D. Yuan, M. A. Omary, M. Haranczyk, B. Smit and H.-C. Zhou, Nat. Commun., 2014, 5, 5723.

219 T. M. McDonald, W. R. Lee, J. A. Mason, B. M. Wiers, C. S. Hong and J. R. Long, J. Am. Chem. Soc., 2012, 134, 7056-7065.

220 D. A. Yang, H. Y. Cho, J. Kim, S. T. Yang and W. S. Ahn, Energy Environ. Sci., 2012, 5, 6465-6473.

221 H. Y. Cho, D. A. Yang, J. Kim, S. Y. Jeong and W. S. Ahn, Catal. Today, 2012, 185, 35-40.

222 L. J. Wang, H. Deng, H. Furukawa, F. Gándara, K. E. Cordova, D. Peri and O. M. Yaghi, Inorg. Chem., 2014, 53, 5881-5883.

223 B. I. Kharisov, O. V. Kharissova and U. O. Méndez, Microwave Hydrothermal and Solvothermal Processing of Materials and Compounds, in The Development and Application of Microwave Heating, ed. W. Cao, InTech, 2012.

224 J. Lhoste, X. Rocquefelte, K. Adil, R. Dessapt, S. Jobic, M. Leblanc, V. Maisonneuve and İ. Bujoli-Doeuff, Inorg. Chem., 2011, 20, 5671-5678.

225 E. Yang, H.-Y. Li, Z.-S. Liu and Q.-D. Ling, Inorg. Chem. Commun., 2013, 30, 152-155. 
226 H. Liu, X. Meng, L. Zhang and A. Jia, Aust. J. Chem., 2015, 68, 1299-1304.

227 Z.-Q. Jiang, G.-Y. Jiang, D.-C. Hou, F. Wang, Z. Zhao and J. Zhang, CrystEngComm, 2013, 15, 315-323.

228 M. Zhu, W. Fu and G. Zou, J. Coord. Chem., 2012, 65, 41084114.

229 S.-Y. Chen, E. Yang, Z.-S. Liu and X.-L. Ye, Chin. J. Struct. Chem., 2012, 31, 535-538.

230 X. Li, Q. Liu and Y. Dong, Synth. React. Inorg., Met.-Org., Nano-Met. Chem., 2016, 46, 1202-1206.

231 H. Fu, C. Qin, Y. Lu, Z.-M. Zhang, Y.-G. Li, Z.-M. Su, W.-L. Li and E.-B. Wang, Angew. Chem., Int. Ed., 2012, 51, 79857989.

232 E. Ahmed and M. Ruck, Angew. Chem., Int. Ed., 2012, 51, 308-323.

233 W.-J. Ji, Q.-G. Zhai, S.-N. Li, Y.-C. Jiang and M.-C. Hu, Inorg. Chem. Commun., 2013, 28, 16-19.

234 A. López-Periago, O. Vallcorba, C. Frontera, C. Domingo and J. A. Ayllón, Dalton Trans., 2015, 44, 7548-7553.

235 J. Gao, M. He, Z. Y. Lee, W. Cao, W.-W. Xiong, Y. Li, R. Ganguly, T. Wuc and Q. Zhang, Dalton Trans., 2013, 42, 11367-11370.

236 S. K. Nune, P. K. Thallapally, B. P. McGrail, H. V. R. Annapureddy, L. X. Dang, D. Mei, N. Karri, K. J. Alvine, M. J. Olszta, B. W. Arey and A. Dohnalkova, ACS Appl. Mater. Interfaces, 2015, 7, 21712-21716.

237 A. Carné-Sánchez, I. Imaz, M. Cano-Sarabia and D. Maspoch, Nat. Chem., 2013, 5, 203-211.

238 H. Furukawa, U. Müller and O. M. Yaghi, Angew. Chem., Int. Ed., 2015, 54, 3417-3430.

239 Y. Sun and B. Yoo, New J. Chem., 2015, 39, 4218-4221.

240 G. Lu, S. Z. Li, Z. Guo, O. K. Farha, B. G. Hauser, X. Y. Qi, Y. Wang, X. Wang, S. Y. Han, X. G. Liu, J. S. DuChene, H. Zhang, Q. C. Zhang, X. D. Chen, J. Ma, S. C. J. Loo, W. D. Wei, Y. H. Yang, J. T. Hupp and F. W. Huo, Nat. Chem., 2012, 4, 310-316.

241 W.-W. Xiong, E. U. Athresh, Y. T. Ng, J. Ding, T. Wu and Q. Zhang, J. Am. Chem. Soc., 2013, 135, 1256-1259.

242 Y.-Y. Yin, J.-G. Ma, Z. Niu, X.-C. Cao, W. Shi and P. Cheng, Inorg. Chem., 2012, 51, 4784-4790.

243 J. Kim, S. H. Kim, S. T. Yang and W. S. Ahn, Microporous Mesoporous Mater., 2012, 161, 48-55.

244 H. Y. Cho, J. Kim, S. N. Kim and W. S. Ahn, Microporous Mesoporous Mater., 2013, 169, 180-184.

245 D. Deng, L. Liu, B.-M. Ji, G. Yin and C. Du, Cryst. Growth Des., 2012, 12, 5338-5348.

246 Z.-Z. Wen, X.-L. Wen, S.-L. Cai, S.-R. Zheng, J. Fan and W.-G. Zhang, CrystEngComm, 2013, 15, 5359-5367.

247 S. Zhong, H. Jing, Y. Li, S. Yin, C. Zeng and L. Wang, Inorg. Chem., 2014, 53, 8278-8286.

248 M. Shi, C. Zeng, L. Wang, Z. Nie, Y. Zhao and S. Zhong, New J. Chem., 2015, 39, 2973-2979.

249 M. Joaristi, J. Juan-Alcaniz, P. Serra-Crespo, F. Kapteijn and J. Gascon, Cryst. Growth Des., 2012, 12, 3489-3498.

250 H. Sakamoto, R. Matsuda and S. Kitagawa, Dalton Trans., 2012, 41, 3956-3961.
251 D. Crawford, J. Casaban, R. Haydon, N. Giri, T. McNally and S. L. James, Chem. Sci., 2015, 6, 1645-1649.

252 D. Matoga, M. Oszajca and M. Molenda, Chem. Commun., 2015, 51, 7637-7640.

253 V. Safarifard and A. Morsali, Coord. Chem. Rev., 2015, 292, 1-14.

254 M. J. S. Fard, F. Rastaghi and N. Ghanbari, J. Mol. Struct., 2013, 1032, 133-143.

255 A. Morsali and A. Panjehpour, Inorg. Chim. Acta, 2012, 391, 210-217.

256 V. Safarifard and A. Morsali, Ultrason. Sonochem., 2012, 19, 300-306.

257 Z. Zhang, W.-Y. Gao, L. Wojtas, S. Ma, M. Eddaoudi and M. J. Zaworotko, Angew. Chem., Int. Ed., 2012, 51, 93309334.

258 J. D. Evans, C. J. Sumby and C. J. Doonan, Chem. Soc. Rev., 2014, 43, 5933-5951.

259 M. Servalli, M. Ranocchiari and J. A. Van Bokhoven, Chem. Commun., 2012, 48, 1904-1906.

260 L. Zhu, X.-Q. Liu, H.-L. Jiang and L.-B. Sun, Chem. Rev., 2017, 117, 8129-8176.

261 B. Gui, X. Meng, H. Xu and C. Wang, Chin. J. Chem., 2016, 34, 186-190.

262 B. Li, B. Gui, G. Hu, D. Yuan and C. Wang, Inorg. Chem., 2015, 54, 5139-5141.

263 P. Deria, J. E. Mondloch, O. Karagiaridi, W. Bury, J. T. Hupp and O. K. Farha, Chem. Soc. Rev., 2014, 43, 5896-5912.

264 H. Wang, W. Meng, J. Wu, J. Ding, H. Hou and Y. Fan, Coord. Chem. Rev., 2016, 307, 130-146.

265 S. Yuan, Y.-P. Chen, J.-S. Qin, W. Lu, L. Zou, Q. Zhang, X. Wang, X. Sun and H.-C. Zhou, J. Am. Chem. Soc., 2016, 138, 8912-8919.

266 C. Montoro, E. Garcia, S. Calero, M. A. Perez-Fernandez, A. L. Lopez, L. Barea and J. A. Navarro, J. Mater. Chem., 2012, 22, 10155-10158.

267 P. V. Dau and S. M. Cohen, Chem. Commun., 2013, 49, 61286130.

268 K. M. Zwoliński, P. Nowak and M. J. Chmielewski, Chem. Commun., 2015, 51, 10030-10033.

269 A. S. Gupta, R. K. Deshpande, L. Liu, G. I. N. Waterhouse and S. G. Telfer, CrystEngComm, 2012, 14, 5701-5704.

270 W. Bury, D. Fairen-Jimenez, M. B. Lalonde, R. Q. Snurr, O. K. Farha and J. T. Hupp, Chem. Mater., 2013, 25, 739744.

271 A. F. Gross, E. Sherman, S. L. Mahoney and J. J. Vajo, J. Phys. Chem. A, 2013, 117, 3771-3781.

272 T. Li, M. T. Kozlowski, E. A. Doud, M. N. Blakely and N. L. Rosi, J. Am. Chem. Soc., 2013, 135, 11688-11691.

273 S. Takaishi, E. J. DeMarco, M. J. Pellin, O. K. Farha and J. T. Hupp, Chem. Sci., 2013, 4, 1509-1513.

274 D. H. Hong and M. P. Suh, Chem.-Eur. J., 2014, 20, 426434.

275 M. Kim, J. F. Cahill, Y. Su, K. A. Prather and S. M. Cohen, Chem. Sci., 2012, 3, 126-130.

276 H. Fei, J. Shin, Y. S. Meng, M. Adelhardt, J. Sutter, K. Meyer and S. M. Cohen, J. Am. Chem. Soc., 2014, 136, 4965-4973. 
277 H. Fei and S. M. Cohen, J. Am. Chem. Soc., 2015, 137, 21912194.

278 C. Liu, T.-Y. Luo, E. S. Feura, C. Zhang and N. L. Rosi, J. Am. Chem. Soc., 2015, 137, 10506-10509.

279 V. Safarifard and A. Morsali, Inorg. Chim. Acta, 2013, 405, 203-208.

280 J. B. Rivest and P. K. Jain, Chem. Soc. Rev., 2013, 42, 89-96. 281 Y. Kim, S. Das, S. Bhattacharya, S. Hong, M. G. Kim, M. Yoon, S. Natarajan and K. Kim, Chem.-Eur. J., 2012, 18, 16642-16648.

282 D. Denysenko, T. Werner, M. Grzywa, A. Puls, V. Hagen, G. Eickerling, J. Jelic, K. Reuter and D. Volkmer, Chem. Commun., 2012, 48, 1236-1238.

283 C. K. Brozek, A. F. Cozzolino, S. J. Teat, Y.-S. Chen and M. Dincă, Chem. Mater., 2013, 25, 2998-3002.

284 Y. Jiao, C. R. Morelock, N. C. Burtch, W. P. Mounfield III, J. T. Hungerford and K. S. Walton, Ind. Eng. Chem. Res., 2015, 54, 12408-12414.

285 A. M. Shultz, A. A. Sarjeant, O. K. Farha, J. T. Hupp and S. T. Nguyen, J. Am. Chem. Soc., 2011, 133, 13252-13255.

286 D. Feng, Z.-Y. Gu, Y.-P. Chen, J. Park, Z. Wei, Y. Sun, M. Bosch, S. Yuan and H.-C. Zhou, J. Am. Chem. Soc., 2014, 136, 17714-17717.

287 J. Zheng, M. Wu, F. Jiang, W. Su and M. Hong, Chem. Sci., 2015, 6, 3466-3470.

288 J. A. Johnson, J. Luo, X. Zhang, Y.-S. Chen, M. D. Morton, E. Echeverría, F. E. Torres and J. Zhang, ACS Catal., 2015, 5, 5283-5291.

289 T. Sawano, N. C. Thacker, Z. Lin, A. R. McIsaac and W. Lin, J. Am. Chem. Soc., 2015, 137, 12241-12248.

290 S. Seth, G. Savitha and J. N. Moorthy, J. Mater. Chem. A, 2015, 3, 22915-22922.

291 M. C. So, S. Jin, H.-J. Son, G. P. Wiederrecht, O. K. Farha and J. T. Hupp, J. Am. Chem. Soc., 2013, 135, 15698-15701.

292 V. Stavila, J. Volponi, A. M. Katzenmeyer, M. C. Dixon and M. D. Allendorf, Chem. Sci., 2012, 3, 1531-1540.

293 A. A. Talin, A. Centrone, A. C. Ford, M. E. Foster, V. Stavila, P. Haney, R. A. Kinney, V. Szalai, F. El Gabaly, H. P. Yoon and M. D. Allendorf, Science, 2014, 343, 66-69.

294 M. Tu, S. Wannapaiboon and R. Fischer, Inorg. Chem. Front., 2014, 1, 442-463.

295 P. Szilágyi, R. Westerwaal, R. van de Krol, H. Geerlings and B. Dam, J. Mater. Chem. C, 2013, 1, 8146-8155.

296 M. Tu and R. A. Fischer, J. Mater. Chem. A, 2014, 2, 20182022.

297 M. C. So, M. H. Beyzavi, R. Sawhney, O. Shekhah, M. Eddaoudi, S. S. Al-Juaid, J. T. Hupp and O. K. Farha, Chem. Commun., 2015, 51, 85-88.

298 Z. Wang, J. Liu, H. K. Arslan, S. Grosjean, T. Hagendorn, H. Gliemann, S. Bräse and C. Wüll, Langmuir, 2013, 29, 15958-15964.

299 R. Das, P. Pachfule, R. Banerjee and P. Poddar, Nanoscale, 2012, 4, 591-599.

300 T.-Y. Ma, L. Liu and Z.-Y. Yuan, Chem. Soc. Rev., 2013, 42, 3977-4003.

301 Q. Tian, Z. Zhang, L. Yang and S. Hirano, Carbon, 2015, 93, 887-895.
302 C. P. Li, J. Chen, C. S. Liu and M. Du, Chem. Commun., 2015, 51, 2768-2781.

303 R. Matsuoka, R. Toyoda, R. Sakamoto, M. Tsuchiya, K. Hoshiko, T. Nagayama, Y. Nonoguchi, K. Sugimoto, E. Nishibori, T. Kawai and H. Nishihara, Chem. Sci., 2015, 6, 2853-2858.

304 W. Xia, A. Mahmood, R. Zou and Q. Xu, Energy Environ. Sci., 2015, 8, 1837-1866.

305 Z. Han, Y. Yu, Y. Zhang, B. Dong, A. Kong and Y. Shan, J. Mater. Chem. A, 2015, 3, 23716-23724.

306 N. L. Torad, M. Hu, S. Ishihara, H. Sukegawa, A. A. Belik, M. Imura, K. Ariga, Y. Sakka and Y. Yamauchi, Small, 2014, 10, 2096-2107.

307 W. Bak, H. S. Kim, H. Chun and W. C. Yoo, Chem. Commun., 2015, 51, 7238-7241.

308 M. Zhang, Y.-L. Huang, J.-W. Wang and T.-B. Lu, J. Mater. Chem. A, 2016, 4, 1819-1827.

309 B. Mirtamizdoust, D. C. Bienko, Y. Hanifehpour, E. R. T. Tiekink, V. T. Yilmaz, P. Talemi and S. W. Joo, J. Inorg. Organomet. Polym., 2016, 26, 819-828.

310 S. Aghabeygi, F. Bigdeli and A. Morsali, J. Inorg. Organomet. Polym., 2012, 22, 526-529.

311 X. Xu, R. Cao, S. Jeong and J. Cho, Nano Lett., 2012, 12, 4988-4991.

312 F. Zhang, D.-D. Qi and X.-G. Zhang, Int. J. Electrochem. Sci., 2016, 11, 189-199.

313 L. Hu, Y. Huang, F. Zhang and Q. Chen, Nanoscale, 2013, 5, 4186-4190.

314 S. Zhang, H. Liu, C. Sun, P. Liu, L. Li, Z. Yang, X. Feng, F. Huo and X. Lu, J. Mater. Chem. A, 2015, 3, 52945298.

315 M. Moeinian and K. Akhbari, J. Inorg. Organomet. Polym., 2016, 26, 1-13.

316 B. Mirtamizdoust, B. Shaabani, A. Khandar, H.-K. Fun, S. Huang, M. Shadman and P. Hojati-Talemi, Z. Anorg. Allg. Chem., 2012, 638, 844-846.

317 Y. Hanifehpour, B. Mirtamizdoust and S. W. Joo, J. Inorg. Organomet. Polym., 2012, 22, 816-821.

318 Y. Hanifehpour, B. Mirtamizdoust, A. R. Farzam and S. W. Joo, J. Inorg. Organomet. Polym., 2012, 22, 957-962.

319 K. Bijanzad, A. Tadjarodi and O. Akhavan, Chin. J. Catal., 2015, 36, 742-749.

320 H. Kim, M. Park, H. Lee and O. S. Jung, Dalton Trans., 2015, 44, 8198-8204.

321 F. Marandi, L. Hashemi, A. Morsali and H. Krautscheid, J. Inorg. Organomet. Polym., 2016, 26, 962-974.

322 V. Safarifard and A. Morsali, Ultrason. Sonochem., 2012, 19, 1227-1233.

323 A. V. Borhadea, B. K. Uphadeb and D. R. Tope, J. Chem. Sci., 2013, 125, 583-589.

324 L. Hashemi, A. Aslani and A. Morsali, J. Inorg. Organomet. Polym., 2012, 22, 867-872.

325 S. Saeednia, P. Iranmanesh, H. A. Rudbari and L. Saeednia, J. Macromol. Sci., Part A: Pure Appl. Chem., 2016, 53, 227236. 
326 R. Gupta, S. Sanotra, H. Nawaz Sheikh, B. Lal Kalsotra, V. Kumar Gupta and Rajnikant, J. Coord. Chem., 2012, 65, 3917-3931.

327 L. Wang, H. Zou, Y. Li, X. Li and S. Zhong, Mater. Manuf. Processes, 2017, 32, 484-488.

328 S. Jin, J. P. Hill, Q. Ji, L. Kumar Shrestha and K. Ariga, J. Mater. Chem. A, 2016, 4, 5737-5746.

329 L. Hashemi and A. Morsali, J. Inorg. Organomet. Polym., 2012, 22, 272-277.

330 M. A. Alavi, A. Morsali, S. W. Joo and B. K. Min, Ultrason. Sonochem., 2015, 22, 349-358.

331 A. Mehrani, A. Morsali, Y. Hanifehpour and S. W. Joo, Ultrason. Sonochem., 2014, 21, 1430-1434.

332 S. Hei, Y. Jin and F. Zhang, J. Chem., 2014, 2014, 546956.

333 F. Meng, Z. Fang, Z. Li, W. Xu, M. Wang, Y. Liu, J. Zhang, W. Wang, D. Zhao and X. Guo, J. Mater. Chem. A, 2013, 1, 7235-7241.

334 N. Nasihat Sheno, A. Morsali and S. Woo Joo, Mater. Lett., 2014, 117, 31-33.

335 M. Hu, A. A. Belik, M. Imura, K. Mibu, Y. Tsujimoto and Y. Yamauchi, Chem. Mater., 2012, 24, 2698-2707.

336 L. Zhang, H. B. Wu, S. Madhavi, H. H. Hng and X. W. Lou, J. Am. Chem. Soc., 2012, 134, 17388-17391.

337 L. Zhang, H. B. Wu, R. Xu and X. W. Lou, CrystEngComm, 2013, 15, 9332-9335.

338 K. J. Lee, T.-H. Kim, T. K. Kim, J. H. Lee, H.-K. Song and H. R. Moon, J. Mater. Chem. A, 2014, 2, 14393-14400.

339 M. Y. Masoomi and A. Morsali, Ultrason. Sonochem., 2016, 28, 240-249.

340 A. Abbasi, M. Gharib and M. Najafi, J. Sci., Islamic Repub. Iran, 2016, 27, 217-221.

341 A. Tahmasian and A. Morsali, Inorg. Chim. Acta, 2012, 387, 327-331.

342 A. Hosseinian, S. Jabbari, A. Reza Mahjoub and M. Movahedi, J. Coord. Chem., 2012, 65, 2623-2633.

343 J. H. Lee, Y. J. Sa, T. K. Kim, H. R. Moon and S. H. Joo, J. Mater. Chem. A, 2014, 2, 10435-10443.

344 H. Jiang, C. Wang, H. Wang and M. Zhang, Mater. Lett., 2016, 168, 17-19.

345 T. K. Kim, K. J. Lee, J. Y. Cheon, J. H. Lee, S. H. Joo and H. R. Moon, J. Am. Chem. Soc., 2013, 135, 8940-8946.

346 Z.-M. Liu, S.-H. Wu, S.-Y. Jia, F.-X. Qin, S.-M. Zhou, H.-T. Ren, P. Na and Y. Liu, Mater. Lett., 2014, 132, 8-10.

347 K. Akhbari, N. B. Bahman, A. Morsali and P. Retailleau, J. Iran. Chem. Soc., 2016, 13, 165-169.
348 S. Y. Moon, M. W. Park, T. H. Noh and O.-S. Jung, J. Mol. Struct., 2013, 1054-1055, 326-332.

349 F. Marandi, L. Hashemi, A. Morsali and H. Krautscheid, Ultrason. Sonochem., 2016, 32, 86-94.

350 F. Shahangi Shirazi and K. Akhbari, Inorg. Chim. Acta, 2015, 436, 1-6.

351 M. Moeinian and K. Akhbari, J. Solid State Chem., 2015, 225, 459-463.

352 A. Mehrani, A. Morsali and P. Ebrahimpour, J. Coord. Chem., 2013, 66, 856-867.

353 G. H. Shahverdizadeh, F. Hakimi, B. Mirtamizdoust, A. Soudi and P. Hojati-Talemi, J. Inorg. Organomet. Polym., 2012, 22, 903-909.

354 B. Mirtamizdoust, B. Shaabani, A. Khandar, H. Pourradi, Y. Abbasityula, H. Goudarziafshar, D. Viterbo, G. Croce and P. Hojati-Talemi, J. Inorg. Organomet. Polym., 2012, 22, 1293-1299.

355 T. Demars, M. Boltoeva, N. Vigier, J. Maynadié, J. Ravaux, C. Genre and D. Meyer, Eur. J. Inorg. Chem., 2012, 2012, 3875-3884.

356 Z. V. Dobrokhotova, N. V. Gogoleva, E. N. ZorinaTikhonova, M. A. Kiskin, V. V. Chernyshev, A. L. Emelina, M. A. Bukov, A. S. Goloveshkin, I. S. Bushmarinov, A. A. Sidorov, A. S. Bogomyakov, M. L. Kovba, V. M. Novotortsev and I. L. Eremenko, Eur. J. Inorg. Chem., 2015, 2015, 3116-3127.

357 A. V. Gavrikov, P. S. Koroteev, Z. V. Dobrokhotova, A. B. Ilyukhin, N. N. Efimov, D. I. Kirdyankin, M. A. Bykov, M. A. Ryumin and V. M. Novotortsev, Polyhedron, 2015, 102, 48-59.

358 N. Zauzolkova, Z. Dobrokhotova, A. Lermontov, E. Zorina, A. Emelina, M. Bukov, V. Chernyshev, A. Sidorov, M. Kiskin, A. Bogomyakov, A. Lytvynenko, S. Kolotilov, Y. Velikodnyi, M. Kovba, V. Novotortsev and I. Eremenko, J. Solid State Chem., 2013, 197, 379-391.

359 M. A. Ryumin, Z. V. Dobrokhotova, A. L. Emelina, M. A. Bukov, N. V. Gogoleva, K. S. Gavrichev, E. N. ZorinaTikhonova, L. I. Demina, M. A. Kiskin, A. A. Sidorov, I. L. Eremenko and V. M. Novotortsev, Polyhedron, 2015, 87, 28-37.

360 D. Hong, Y. Yamada, M. Sheehan, S. Shikano, C.-H. Kuo, M. Tian, C.-K. Tsung and S. Fukuzumi, ACS Sustainable Chem. Eng., 2014, 2, 2588-2594.

361 Y. Xia, B. Wang, G. Wang, X. Liu and H. Wang, ChemElectroChem, 2016, 3, 299-308. 